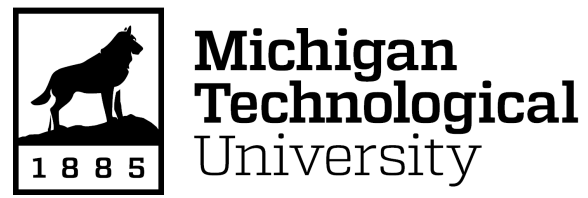

Michigan Technological University Digital Commons @ Michigan Tech

Dissertations, Master's Theses and Master's Reports

2021

\title{
MATHEMATICAL MODELING AND NUMERICAL SIMULATION OF HEAT TRANSFER FROM ISOLATED OBJECTS
}

Esmaeil Dehdashti

Michigan Technological University, dehdasht@mtu.edu

Copyright 2021 Esmaeil Dehdashti

\section{Recommended Citation}

Dehdashti, Esmaeil, "MATHEMATICAL MODELING AND NUMERICAL SIMULATION OF HEAT TRANSFER FROM ISOLATED OBJECTS", Open Access Dissertation, Michigan Technological University, 2021.

https://doi.org/10.37099/mtu.dc.etdr/1231 
MATHEMATICAL MODELING AND NUMERICAL SIMULATION OF HEAT TRANSFER FROM ISOLATED OBJECTS

By

Esmaeil Dehdashti

\begin{abstract}
A DISSERTATION
Submitted in partial fulfillment of the requirements for the degree of DOCTOR OF PHILOSOPHY

In Mechanical Engineering-Engineering Mechanics

MICHIGAN TECHNOLOGICAL UNIVERSITY
\end{abstract}

2021

C) 2021 Esmaeil Dehdashti 
This dissertation has been approved in partial fulfillment of the requirements for the Degree of DOCTOR OF PHILOSOPHY in Mechanical Engineering-Engineering Mechanics.

Department of Mechanical Engineering-Engineering Mechanics
Dissertation Advisor: Dr. Hassan Masoud
Committee Member: $\quad$ Dr. Fernando L. Ponta
Committee Member: Dr. Kazuya Tajiri
Committee Member: Dr. Cécile Piret
Department Chair: Dr. William W. Predebon 


\section{Contents}

List of Figures $\ldots \ldots \ldots \ldots \ldots \ldots \ldots$ vi

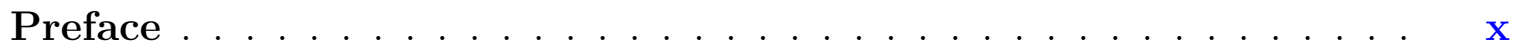

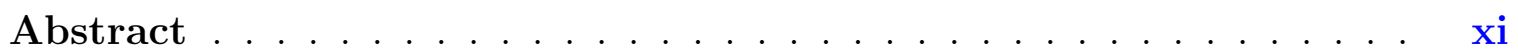

1 Background and significance $\ldots \ldots \ldots \ldots \ldots \ldots \ldots$

1.1 Introduction $\ldots \ldots \ldots \ldots \ldots \ldots \ldots \ldots \ldots \ldots$

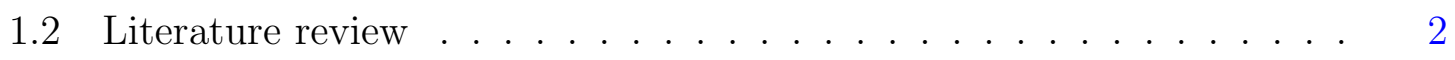

2 Conduction heat transfer from oblate spheroids and bispheres . . 6

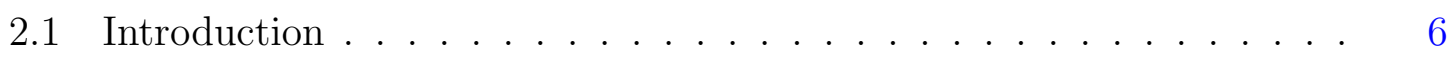

2.2 Problem formulation and solutions $\ldots \ldots \ldots \ldots \ldots$

2.2.1 Temperature field around an oblate spheroid . . . . . . . 8

2.2.2 Temperature field around two identical spheres . . . . . . . 13

2.3 Results and discussion $\ldots \ldots \ldots \ldots \ldots \ldots \ldots$

2.4 Summary . . . . . . . . . . . . . . . . . . . . . 22

3 Forced convection heat transfer from a particle at small and large

Péclet numbers . . . . . . . . . . . . . . . . . 24 
3.1 Introduction . . . . . . . . . . . . . . . . . 24

3.2 Problem statement . . . . . . . . . . . . . . . 26

3.3 Perturbation solution in the limit of conduction-dominated heat trans-

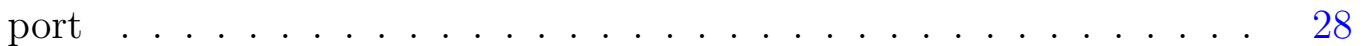

3.4 Perturbation solution in the limit of advection-dominated heat transport 33

3.5 Specific results for spheroids in axisymmetric Stokes flow . . . . . . . 40

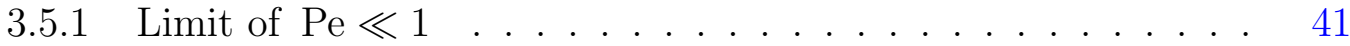

3.5.2 Limit of $\mathrm{Pe} \gg 1$. . . . . . . . . . . . . . . . 42

3.5.3 Comparison with direct numerical solutions . . . . . . . . 45

3.6 Summary . . . . . . . . . . . . . . . . . . . . 47

4 Heat transfer from a particle in laminar flows of a variable thermal conductivity fluid . . . . . . . . . . . . . . . 50

4.1 Introduction . . . . . . . . . . . . . . . . 50

4.2 Problem statement . . . . . . . . . . . . . . . . . 52

4.3 Variation of Nusselt number for constant temperature boundary con-

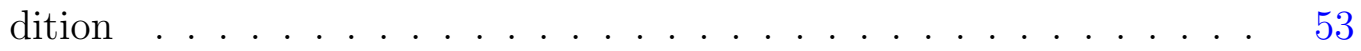

4.3.1 Limit of conduction-dominated heat transport . . . . . . . . . 54

4.3.2 Limit of advection-dominated heat transport . . . . . . . . . 59

4.3.3 Bridging results for limits of low and high Péclet numbers . . 65

4.4 Variation of Nusselt number for uniform heat flux boundary condition 66

4.4.1 Limit of conduction-dominated heat transport . . . . . . . . . 67 
4.4.2 Limit of advection-dominated heat transport . . . . . . . . . . 69

4.4.3 Bridging results for limits of low and high Péclet numbers . . 70

4.5 Comparison with direct numerical solutions . . . . . . . . . . 73

4.6 Summary . . . . . . . . . . . . . . . . . . 75

5 Summary and future directions . . . . . . . . . . . . . 76

Bibliography ...................... 78 


\section{List of Figures}

1.1 Steady-state temperature distribution around a hot particle in uniform Stokes flow at (a) low and (b) high Péclet numbers. The black lines and arrows show the flow streamlines and flow direction, respectively.

2.1 (a) Oblate spheroidal coordinates in a meridian plane. The arrows show the direction of the unit vectors $\boldsymbol{e}_{\xi}$ and $\boldsymbol{e}_{\eta}$. (b) Surfaces of constant $\xi$ and $\eta$ depicted in cyan and gray, respectively. The red curve in (a) and its corresponding surface in (b) represent a hot/cold oblate spheroid that releases/absorbs heat at a constant uniform rate. . . . . . . .

2.2 (a) Bispherical coordinates in a meridian plane. The arrows show the direction of the unit vectors $\boldsymbol{e}_{\zeta}$ and $\boldsymbol{e}_{\beta}$. (b) Surfaces of constant $\zeta$ and $\beta$ depicted in cyan and gray, respectively. The red curves in (a) and their corresponding surfaces in (b) represent a pair of identical hot/cold spheres that release/absorb heat at a constant uniform rate. . . . . .

2.3 (a)-(b) Contour plots of the (dimensionless) temperature field $\theta$ (in meridian planes) around an oblate spheroid of aspect ratio $\varepsilon=0.3$. The left panel illustrates the results of the two-term approximation 
whereas the right panel presents those obtained by setting $m=19$, considered here as exact results. (c) The Nusselt number $\mathrm{Nu}$ versus the aspect ratio $\varepsilon$ for oblate spheroids. Red, blue, and black lines represent the results corresponding to $m=3$, linear interpolation based on the $\mathrm{Nu}$ of sphere and disk, and $m=19$, respectively. The inset shows the relative difference between the approximate and exact results. . . . .

2.4 (a)-(b) Contour plots of the (dimensionless) temperature field $\theta$ (in meridian planes) around two spheres whose centers are three radii apart $(\epsilon=2 / 3)$. The left panel illustrates the results of the four-term approximation whereas the right panel presents those obtained by setting $m=9$, considered here as exact results. (c) $B_{0}$ as a function of $1-\epsilon$, where the dashed and dotted lines represent the asymptotic approximations in the limits of $\epsilon \rightarrow 0$ and $\epsilon \rightarrow 1$, respectively. And, the inset shows the relative error of the approximations. (d) The Nusselt number $\mathrm{Nu}$ versus the inverse of the dimensionless separation distance $\epsilon$ for a pair of identical spheres. Red, blue, green, and black lines represent the results corresponding to $m=3$, linear interpolation based on the $\mathrm{Nu}$ of single sphere and two touching spheres, asymptotic behavior in the limit of $\epsilon \rightarrow 0$, and the converged solution (i.e., $m \gg 1$ ), respectively. The inset shows the relative difference between the approximate and exact results. . . . . . . . . . . . . . . . 
3.1 Schematic of a stationary particle of arbitrary shape, with surface $S_{p}$ and unit outward normal vector $\boldsymbol{n}$, in a uniform fluid flow. The dashed line indicates an enclosing boundary in the far field. . . . . . . .

3.2 Schematic of uniform flow past an axisymmetric heated object at high Péclet number. The boundary layer coordinates are represented by $\mathrm{x}$ and $\mathrm{y}$, and the surface heat flux is denoted by $q \ldots \ldots \ldots$

3.3 Numerically calculated plots of the Nusselt number versus Péclet number for forced convection heat transfer from spheroids of various aspect ratios in an axisymmetric uniform Stokes flow subject to constant heat flux (solid lines) and isothermal (dashed lines) boundary conditions on the surface of the spheroid. . . . . . . . . . . . .

3.4 Percent difference $\Delta$ between the numerical and approximate asymptotic results for the Nusselt number corresponding to (a)-(b) constant heat flux and (c)-(d) isothermal boundary conditions. (a)-(c) and (b)-(d) present $\Delta$ versus Pe curves for approximations based on Eqs. (3.53) and (3.54), respectively. The cut-off Péclet numbers for the aspect ratios $\varepsilon=0.2,0.5,1,2,5$ in (a) and (c) are, respectively, $\mathrm{Pe}_{c}=0.2,1,1,0.35,0.25$ and $\mathrm{Pe}_{c}=1,1,1,0.35,0.25 \ldots \ldots . . . . \quad 48$

4.1 Percent difference $\Delta$ between the results of full numerical simulations and the predictions of Eq. (4.56) for $\mathrm{Nu}_{T}$. . . . . . . . . . . 71

4.2 Percent difference $\Delta$ between the results of full numerical simulations 
and the predictions of Eq. (4.71) for $\mathrm{Nu}_{Q} \cdot \ldots \ldots \ldots \ldots$ 


\section{Preface}

This dissertation includes contents that were previously published as journal articles, whose full citations are:

- Conduction heat transfer from oblate spheroids and bispheres, S. Jafari Kang, E. Dehdashti, and H. Masoud, International Journal of Heat and Mass Transfer 139, 115-120 (2019);

- Forced convection heat transfer from a particle at small and large Peclet numbers, E. Dehdashti and H. Masoud, Journal of Heat Transfer 142, 061803 (2020);

- Heat transfer from a particle in laminar flows of a variable thermal conductivity fluid, E. Dehdashti, M. Razizadeh, and H. Masoud, International Journal of Heat and Mass Transfer 171, 121067 (2021).

As evidenced from the list of authors, the first and last publications were collaborative works involving, respectively, Saeed Jafari Kang and Meghdad Razizadeh. In the first collaborative study, all authors contributed to the theoretical analyses, numerical simulations, and writing of the manuscript. And, in the second one, the primary contributors were the author of this dissertation (Esmaeil Dehdashti) and his advisor (Dr. Hassan Masoud), with Meghdad Razizadeh being only involved in some early analysis. 


\section{Abstract}

In the area of heat transfer, like other fields of science and engineering, full- and semi-analytical solutions of elementary problems are regarded as invaluable resources that can be used to identify relevant dimensionless parameters, to obtain basic insights into the phenomena under consideration, to quickly quantify the effects of key factors, and, ultimately, to pave the way for understanding more complex problems arising in practice. These solutions can also serve as excellent benchmarks for calibrating experimental setups and validating numerical techniques.

In this dissertation, we theoretically study three classical heat transfer problems, with the ultimate goal of deriving analytical or approximate expressions for the Nusselt number (denoted by $\mathrm{Nu}$ ), which is a key dimensionless parameter that quantifies the transfer of heat to and from a surface. First, we consider heat transfer by conduction from oblate spheroidal and bispherical surfaces into a stationary, infinite medium. The surfaces are presumed to maintain a constant heat flux. Assuming steady-state condition and uniform thermal conductivity, we analytically solve the Laplace equation for the temperature distribution and discuss the challenge of dealing with the Neumann (uniform flux) versus more convenient Dirichlet (isothermal) boundary condition. The solutions are obtained in boundary-fitting coordinate systems using the method of separation of variables and eigenfunction expansion. And, exact expressions for the average Nusselt number are presented along with their approximations.

Next, we examine forced convection heat transfer from a single particle in uniform 
laminar flows. Asymptotic limits of small and large Péclet numbers (denoted by Pe) are considered. For $\mathrm{Pe} \ll 1$ (diffusion-dominated regime) and a constant heat flux boundary condition on the surface of the particle, we obtain a closed-form expression for the heat transfer coefficient that is valid for arbitrary particle shapes and Reynolds numbers, as long as the flow is incompressible. We also present a framework for calculating the average Nusselt number of axisymmetric and two-dimensional objects with a constant heat flux surface condition in the limits of $\mathrm{Pe} \gg 1$ and small or moderate Reynolds numbers. Specific results are given for the heat transfer from spheroidal particles in Stokes flow.

Finally, we revisit the problem of steady-state heat transfer from a single particle in a uniform laminar flow with the assumption that the thermal conductivity of the fluid changes linearly with the temperature. We use a combination of asymptotic and scaling analyses to derive approximate expressions for the Nusselt number of arbitrarily shaped particles. The results cover the entire range of the Péclet number. We find that, for a constant temperature boundary condition and fixed geometry, the Nusselt number is essentially equal to the product of two terms, one of which is only a function of Pe while the other one is nearly independent of Pe and mainly depends on the proportionality constant of the conductivity-temperature relation. We also show that, in contrast, when a uniform heat flux is imposed on the surface of the particle, the Nusselt number can be estimated as a summation of a Pe-dependent piece and one that solely varies with the proportionality constant. 


\section{Chapter 1: Background and significance}

\subsection{Introduction}

Fundamental problems in any field of science and engineering are the foundational blocks upon which the rest of the filed is formed. Often, complex phenomena and intricate systems are interpreted and analyzed through the lens of these basic problems, which highlights their importance in advancing the state-of-the-art and in making new discoveries. This is certainly the case in thermal sciences, which among other topics, deals with the transfer of thermal energy (i.e., heat) between objects and systems.

In the area of heat transfer, a subcategory is devoted to the exchange of heat between small objects (i.e., particles) and their surrounding medium. The principal objective of this $\mathrm{Ph} . \mathrm{D}$. research is to fundamentally contribute to this branch of heat transfer through theoretical examination of (i) conduction heat transfer from oblate spheroids and bispheres, (ii) forced convection heat transfer from an arbitrarilyshaped particle, and (iii) heat transfer from a single object in laminar flows of a variable thermal conductivity fluid.

This dissertation is organized as follows. In the rest of this introductory chapter, we put our work in context by reviewing some of the classical theoretical investigations 

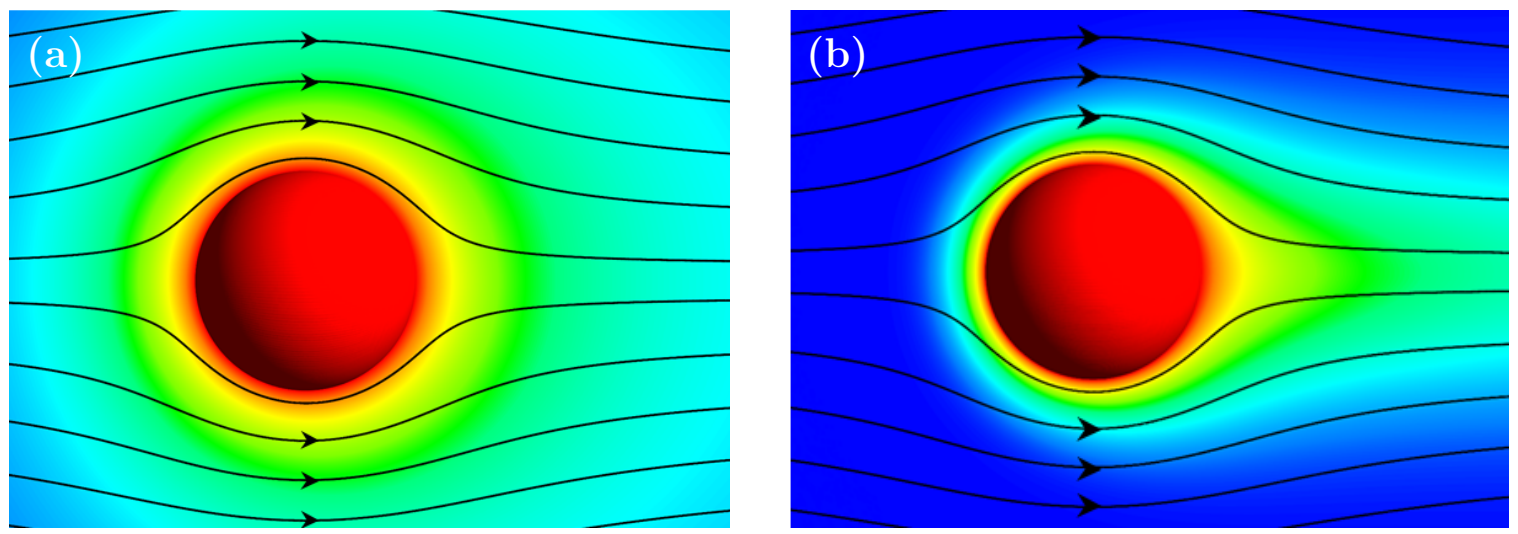

Figure 1.1: Steady-state temperature distribution around a hot particle in uniform Stokes flow at (a) low and (b) high Péclet numbers. The black lines and arrows show the flow streamlines and flow direction, respectively.

on conduction and convection heat transfer from particles. In the next three chapters, we discuss the details of our analysis for each of the above-mentioned problems. And, in the last chapter, we summarize our study and give a few ideas for future directions.

\subsection{Literature review}

Convection heat transfer problems are commonly classified in terms of a dimensionless parameter called the Péclet number (denoted by Pe), which measures the importance of advective heat transport relative to conductive transport of the thermal energy. According to this definition, $\mathrm{Pe}=0$ represents a class of problems known as pure conduction. When the Péclet number is very small $(\mathrm{Pe} \ll 1)$, but finite, the transfer of heat is still dominated by conduction (see, e.g., Fig. 1.1a). However, as Pe rises, the influence of advection grows and eventually becomes comparable with that of conduction when $\mathrm{Pe} \sim \mathcal{O}(1)$. With further increase of the Péclet number, especially beyond $\mathcal{O}\left(10^{2}\right)$, advection becomes the dominant mode of heat transfer and conduction 
will be limited to a narrow region (known as the boundary layer) close to the surface of the particle (see, e.g., Fig. 1.1b).

Theoretical study of convection heat transfer at arbitrary Péclet number is often very challenging, if not impossible. As a result, problems treated in the vast majority of theoretical investigations correspond to either $\mathrm{Pe}=0$ or the asymptotic limits of Pe (i.e., $\mathrm{Pe} \ll 1$ and $\mathrm{Pe} \gg 1$ ) [1-16]. The latter choice makes the problem amenable to the perturbation theory, using which very useful approximations can be derived for the quantities of interest. One of such quantities is the dimensionless rate of total heat transfer from a surface, which is known as the Nusselt number and is denoted by $\mathrm{Nu}$. In the following and beginning with the case of $\mathrm{Pe}=0$, we summarize several classical studies where $\mathrm{Nu}$ of an object is theoretically calculated for a particular Pe regime under steady-state condition. We note that this review is not meant to be comprehensive.

The literature on pure conduction is vast and most of the studies on this subject center around the solution of the Laplace equation for the temperature distribution. Once the temperature field is known, then the integral of the local heat flux on the surface of the object is calculated to obtain the Nusselt number. Among many other studies, Yovanovich [17] and Hahne and Grigull [18] analytically solved for and catalogued $\mathrm{Nu}$ for a number of practical geometries. The Nusselt number for additional geometries are reported in classical heat transfer textbooks (see, e.g., [19]), with references given to the original derivations. Furthermore, Brenner and Haber 
[20] presented a general solution for the Laplace equation, using the symbolic operator method, that is valid for a general far-filed temperature distribution and is not restricted to the typical uniform temperature assumption at infinity. It is worth noting that the boundary value problems associated with pure conduction appear in other fields such as electrostatics as well. And, they are often discussed in mathematical physics textbooks.

In the limit of $\mathrm{Pe} \ll 1$, Brenner [4] showed, in his seminal work, that the leadingorder contribution of advection to the Nusselt number for the problem of uniform flow past an arbitrarily-shaped particle can be calculated by only knowing the conduction Nusselt number of the same problem. This surprisingly general result was obtained by using a singular perturbation expansion for the temperature and by the application of the reciprocal theorem. Remarkably, he proved that the first-order correction to $\mathrm{Nu}$ of pure conduction does not even depend on the details of the flow field. In the opposite limit of $\mathrm{Pe} \gg 1$, Acrivos and Goddard [6, 7] generalized the work of Lighthill [1] in their two-part study, where they developed a framework for calculating the Nusselt number in cases of planar and axisymmetric laminar flows. These asymptotic results for small and large Péclet number were later extended by [21] to problems with variable thermal conductivity.

Overall, our survey of the literature on conduction and convection heat transfer from isolated objects reveals that while there exists a great body of theoretical analyses, there are certain aspects that have received less attention. In particular, we 
have noticed that the boundary conditions considered on the surface of the objects have been limited, to a large extent, to the prescribed temperature condition. And, therefore, there is a need for more extensive examinations of other boundary condition. The theoretical calculations detailed in the subsequent chapters are performed in response to this need. 


\section{Chapter 2: Conduction heat transfer from oblate spheroids and bispheres}

\subsection{Introduction}

In this chapter, we present analytical solutions for the problem of steady-state conduction heat transfer in an infinite medium due to the presence of hot/cold inclusions. Specifically, two geometries for the heat source/sink are considered, namely an oblate spheroid and a pair of spheres (see Figs. 2.1 and 2.2). For both cases, a uniform heat flux is assumed to emanate from the surface of the inclusions. This boundary condition models many scenarios that appear frequently in engineering applications, such as when a surface is covered by a thin layer of electric heater [19]. The assumption of the Neumann boundary condition is the novel aspect of our work. In the following, we first formulate the problem mathematically and then provide a detailed description of the solutions for the two geometries of interest. Next, we discuss the results for the temperature distribution and average Nusselt number and, finally, we give a brief summary of our study. 


\subsection{Problem formulation and solutions}

Consider one or more objects surrounded by an infinite medium at rest. Suppose that heat is released/absorbed from/by the surface of the objects at a constant uniform rate and that the temperature at infinity is maintained at a constant value. Of interest, here, is the steady-state temperature distribution in the surrounding medium assuming that the transport of heat is dominated by conduction and that the thermal conductivity is constant. Given the above conditions, the boundary-value problem governing the distribution of the temperature is

$$
\begin{aligned}
& \nabla^{2} T^{\star}=0 \text { with } \\
& -k \boldsymbol{n} \cdot \boldsymbol{\nabla} T^{\star}=q_{s} \text { for } \boldsymbol{r} \in S_{p} \text { and } T^{\star} \rightarrow T_{\infty}^{\star} \text { as } r \rightarrow \infty,
\end{aligned}
$$

where $T^{\star}$ represents the temperature field, $k$ is the thermal conductivity of the medium, $\boldsymbol{n}$ is the unit vector outward normal to the surface of the objects denoted by $S_{p}, \boldsymbol{r}$ is the position vector with magnitude $r=|\boldsymbol{r}|$, and $q_{s}$ and $T_{\infty}^{\star}$ are constants.

Let $\ell$ be a characteristic length scale of the problem. Then, upon the change of variables $T=k\left(T^{\star}-T_{\infty}^{\star}\right) / q_{s} \ell, \tilde{\boldsymbol{r}}=\boldsymbol{r} / \ell$, and $\tilde{r}=r / \ell$, Eq. (2.1) simplifies to the following dimensionless form:

$$
\begin{aligned}
& \nabla^{2} T=0 \text { with } \\
& \boldsymbol{n} \cdot \boldsymbol{\nabla} T=-1 \text { for } \tilde{\boldsymbol{r}} \in S_{p} \text { and } T \rightarrow 0 \text { as } \tilde{r} \rightarrow \infty
\end{aligned}
$$

One can define an average Nusselt number for this problem as 


$$
\mathrm{Nu}=\frac{\mathbb{S}_{p}}{2 \pi \bar{T}}
$$

where $\mathbb{S}_{p}$ represents the dimensionless surface area of the object and $\bar{T}$ is the mean value of $T$ on $S_{p}$. Below, we solve Eq. (2.2) and calculate the average temperature for

cases in which $S_{p}$ represents the surface of an oblate spheroid and a pair of spheres, respectively. For each case, the solution is obtained via the method of separation of variables and eigenfunction expansion in a coordinate system that matches the boundaries of the problem.

The correctness of the derivations is independently verified by comparing the results against those obtained from the numerical solution of Eq. (2.2). A secondorder finite volume method as implemented in OpenFOAM (see, e.g., [22]) is used to perform the computations. The outer boundary at infinity is modeled as a very large sphere, and 2D axisymmetric meshes concentrated around $S_{p}$ are employed to discretize the physical domains.

\subsubsection{Temperature field around an oblate spheroid}

Consider an oblate spheroid of equatorial radius $\ell$ and aspect ratio (ratio of polar to equatorial radius $) \varepsilon$. Let $(x, y, z)$ be the components of a reference Cartesian coordinate system located at the center of the spheroid such that the $z$ axis coincides with the revolution axis of the spheroid. The coordinates are nondimentionalized by $\ell$. To solve Eq. (2.2), we adopt an oblate spheroidal coordinate system $(\xi, \eta, \varphi)$ defined as 


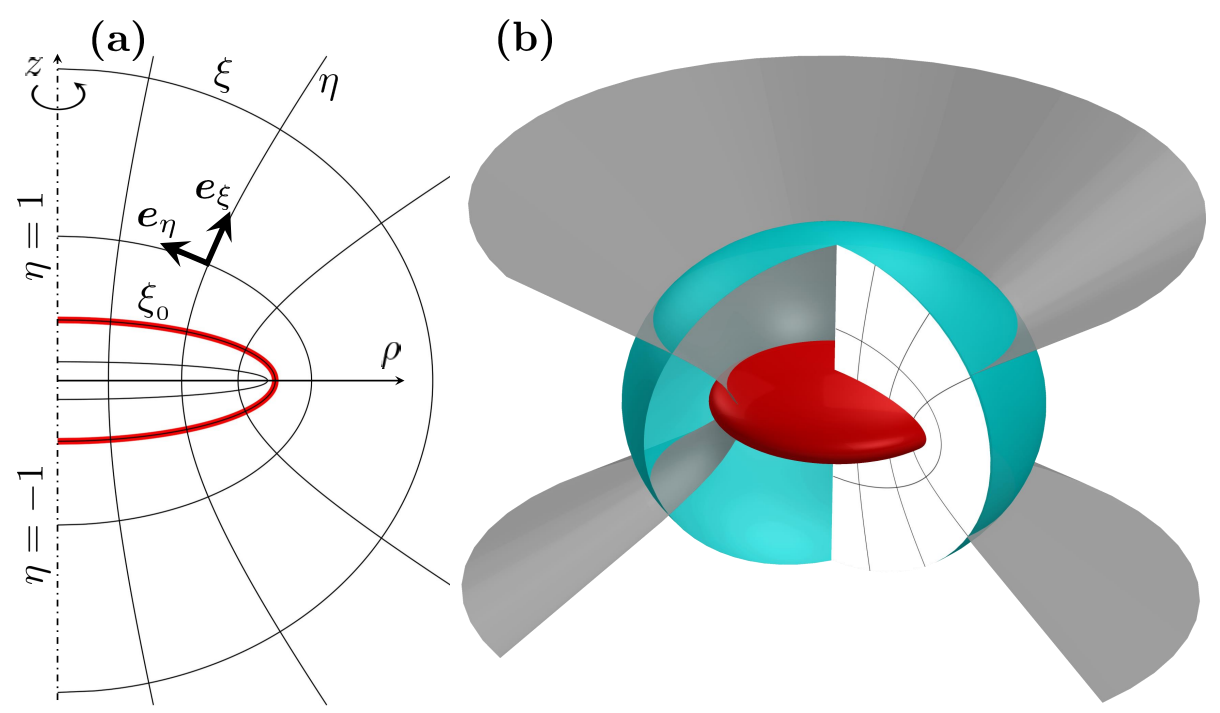

Figure 2.1: (a) Oblate spheroidal coordinates in a meridian plane. The arrows show the direction of the unit vectors $\boldsymbol{e}_{\xi}$ and $\boldsymbol{e}_{\eta}$. (b) Surfaces of constant $\xi$ and $\eta$ depicted in cyan and gray, respectively. The red curve in (a) and its corresponding surface in (b) represent a hot/cold oblate spheroid that releases/absorbs heat at a constant uniform rate.

$[23,24]$

$$
x / \cos \varphi=y / \sin \varphi=c \sqrt{\left(1+\xi^{2}\right)\left(1-\eta^{2}\right)}, \quad z=c \xi \eta
$$

where $c=\sqrt{1-\varepsilon^{2}}$ is the (dimensionless) radius of the focal circle and

$$
0 \leq \xi<\infty, \quad-1 \leq \eta \leq 1, \quad 0 \leq \varphi<2 \pi
$$

The metric coefficients associated with $(\xi, \eta, \varphi)$ are

$$
h_{\xi}=c \sqrt{\frac{\xi^{2}+\eta^{2}}{1+\xi^{2}}}, \quad h_{\eta}=c \sqrt{\frac{\xi^{2}+\eta^{2}}{1-\eta^{2}}}, \quad h_{\varphi}=\rho=\sqrt{x^{2}+y^{2}}
$$

As shown in Fig. 2.1, the surfaces of constant $\xi$ and $\eta$ are oblate spheroids and onesheet hyperboloids of revolution, respectively. In particular, $\xi=\xi_{0}=\varepsilon / \sqrt{1-\varepsilon^{2}}$ represents $S_{p}$ and $\xi \rightarrow \infty$ corresponds to a bounding surface at large distances. 
In the orthogonal curvilinear coordinate system $(\xi, \eta, \varphi)$, Eq. (2.2) takes the form of $[23,24]$

$$
\begin{aligned}
\nabla^{2} T & =\frac{1}{c^{2}\left(\xi^{2}+\eta^{2}\right)}\left\{\frac{\partial}{\partial \xi}\left[\left(1+\xi^{2}\right) \frac{\partial T}{\partial \xi}\right]+\frac{\partial}{\partial \eta}\left[\left(1-\eta^{2}\right) \frac{\partial T}{\partial \eta}\right]\right\} \\
& +\frac{1}{c^{2}\left(1+\xi^{2}\right)\left(1-\eta^{2}\right)} \frac{\partial^{2} T}{\partial \varphi^{2}}=0 \text { with } \\
& \left.\frac{1}{h_{\xi}} \frac{\partial T}{\partial \xi}\right|_{\xi=\xi_{0}}=-1 \text { and } T \rightarrow 0 \text { as } \xi \rightarrow \infty
\end{aligned}
$$

Since the boundary conditions do not depend on $\varphi$, we deduce that $T$ is azimuthally independent, i.e., $\partial T / \partial \varphi=0$. Hence, Laplace's equation for $T$ reduces to

$$
\frac{\partial}{\partial \xi}\left[\left(1+\xi^{2}\right) \frac{\partial T}{\partial \xi}\right]+\frac{\partial}{\partial \eta}\left[\left(1-\eta^{2}\right) \frac{\partial T}{\partial \eta}\right]=0
$$

Starting with the ansatz $T(\xi, \eta)=\mathscr{X}(\xi) \mathscr{H}(\eta)$, where $\mathscr{X}$ and $\mathscr{H}$ are to-be-determined functions, and following the steps involved in the separation of variables technique $[23,24]$, it can be shown that the general solution of Eq. (2.8) is

$$
T=\sum_{m=0}^{\infty}\left[A_{m}^{1} P_{m}(i \xi)+A_{m}^{2} Q_{m}(i \xi)\right]\left[A_{m}^{3} P_{m}(\eta)+A_{m}^{4} Q_{m}(\eta)\right]
$$

where $m$ is an integer, $A_{m}^{1}, \ldots, A_{m}^{4}$ are constants, $i^{2}=-1$, and $P_{m}$ and $Q_{m}$ are Legendre functions of the first and second kind, respectively [25]. The latter function is defined as

$$
\begin{aligned}
Q_{0}(\mathbf{z}) & =\frac{1}{2} \ln \left(\frac{\mathbf{z}+1}{\mathbf{z}-1}\right), \quad Q_{1}(\mathbf{z})=\frac{\mathbf{z}}{2} \ln \left(\frac{\mathbf{z}+1}{\mathbf{z}-1}\right)-1, \\
Q_{n+1}(\mathbf{z}) & =\frac{(2 n+1) \mathbf{z} Q_{n}-n Q_{n-1}}{n+1} .
\end{aligned}
$$

The function $P_{m}(i \xi)$ blows up as $\xi \rightarrow \infty$ for $m \neq 0$ and so does $Q_{m}(\eta)$ at $\eta=$ 
\pm 1 for all $m$. Therefore, to keep the solution finite, we need to set $A_{m}^{1}=0$ for $m \neq 0$ and $A_{m}^{4}=0$ for all $m$. By demanding $T$ to vanish at infinity, we find that $A_{0}^{1}$ is zero, too, since $P_{0}(i \xi)=P_{0}(\eta)=1$ and $Q_{m}(i \xi)$ decays to zero for large $\xi$. Thus, Eq. (2.9) simplifies to

$$
T=\sum_{m=0}^{\infty} A_{m} Q_{m}(i \xi) P_{m}(\eta)
$$

where the constant coefficients $A_{m}$ are determined by applying the constant flux boundary condition on $S_{p}$ :

$$
\left.\frac{1}{h_{\xi}} \frac{\partial T}{\partial \xi}\right|_{\xi=\xi_{0}}=\frac{i}{c} \sqrt{\frac{1+\xi_{0}^{2}}{\xi_{0}^{2}+\eta^{2}}} \sum_{m=0}^{\infty} A_{m} Q_{m}^{\prime}\left(i \xi_{0}\right) P_{m}(\eta)=-1,
$$

with $Q_{m}^{\prime}(\mathfrak{x})=\mathrm{d} Q_{m}(\mathfrak{x}) / \mathrm{d} \mathfrak{x}$. Note that although the gradient of $T$ in the direction normal to the boundary is constant, its derivative with respect to $\xi$ (coordinate normal to the boundary) is equal to the scale factor $h_{\xi}$, which varies along the boundary. Had we been considering the constant temperature boundary condition (i.e., $T=1$ ) at $\xi=\xi_{0}$, we would have been dealing with a significantly simpler situation where $T$ would have been also independent of $\eta$ and Eq. (2.8) would have further reduced to an ordinary differential equation with the solution (see, e.g., [26])

$$
T=\frac{\cot ^{-1} \xi}{\cot ^{-1} \xi_{0}}=\frac{\cot ^{-1} \xi}{\cos ^{-1} \varepsilon} .
$$

The eigenfunction $P_{m}$ has the following orthogonality property:

$$
\int_{-1}^{1} P_{m}(\mathfrak{x}) P_{n}(\mathfrak{x}) \mathrm{d} \mathfrak{x}=\left\{\begin{array}{ccc}
2 /(2 m+1) & \text { if } & n=m \\
0 & \text { if } & n \neq m
\end{array},\right.
$$


where $n$ is an integer. Using this feature, the unknown coefficients are obtained as

$$
A_{m}=\frac{i}{Q_{m}^{\prime}\left(i \xi_{0}\right)} \frac{2 m+1}{2\left(1+\xi_{0}^{2}\right)} \int_{-1}^{1} P_{m}(\eta) \sqrt{\xi_{0}^{2}+\eta^{2}} \mathrm{~d} \eta
$$

The integral in Eq. (2.15) is zero for odd values of $m$ and is otherwise calculated via $[27]$

$$
\begin{aligned}
& \int_{-1}^{1} P_{m}(\eta) \sqrt{\xi_{0}^{2}+\eta^{2}} \mathrm{~d} \eta \\
& =2^{m} \sum_{n=0}^{m / 2} \frac{\Gamma(m / 2+n+1 / 2) I_{2 n}\left(\xi_{0}\right)}{\Gamma(2 n+1) \Gamma(m-2 n+1) \Gamma(n-m / 2+1 / 2)},
\end{aligned}
$$

where $\Gamma$ is the gamma function and

$$
I_{2 n}\left(\xi_{0}\right)=\int_{-1}^{1} \eta^{2 n} \sqrt{\xi_{0}^{2}+\eta^{2}} \mathrm{~d} \eta=\frac{2 \xi_{0}}{2 n+1}{ }_{2} \mathrm{~F}_{1}\left(-1 / 2, n+1 / 2 ; n+3 / 2 ;-\xi_{0}^{-2}\right) .
$$

Here, ${ }_{2} \mathrm{~F}_{1}$ is the hypergeometric function [25]. Having determined the temperature distribution, the average $T$ on the surface of the spheroid can be calculated as

$$
\begin{aligned}
\bar{T} & =\frac{1}{\mathbb{S}_{p}} \int_{S_{p}} T \mathrm{~d} S=\frac{2 \pi c^{2}}{\mathbb{S}_{p}} \sqrt{1+\xi_{0}^{2}} \int_{-1}^{1} T \sqrt{\xi_{0}^{2}+\eta^{2}} \mathrm{~d} \eta \\
& =-\frac{4 \pi \sqrt{1+\xi_{0}^{2}}}{\mathbb{S}_{p}} \sum_{m=0}^{\infty} \frac{A_{m}{ }^{2}}{2 m+1} i Q_{m}^{\prime}\left(i \xi_{0}\right) Q_{m}\left(i \xi_{0}\right),
\end{aligned}
$$

where

$$
\mathbb{S}_{p}=\int_{S_{p}} 1 \mathrm{~d} S=2 \pi\left(1+\frac{\xi_{0}^{2}}{\sqrt{1+\xi_{0}^{2}}} \operatorname{coth}^{-1} \sqrt{1+\xi_{0}^{2}}\right) .
$$

It is worth noting that the results of this section for an oblate spheroid can be converted to those for a prolate spheroid by allowing $\varepsilon$ to be greater than one and by replacing $\xi$ with $-i \xi$ in all relations (see, e.g., [28]). 


\subsubsection{Temperature field around two identical spheres}

Consider a pair of identical spheres, with radius $\ell$, that are placed distance $2 \ell / \epsilon$ apart, where $0<\epsilon<1$. Recall the Cartesian coordinate system of $\S 2.2 .1$. Only this time, the origin is located in the middle of the line that connects the center of the spheres and the $z$ axis is oriented in the direction of that line. The natural coordinate system for dealing with this geometry is a bispherical coordinate system $(\zeta, \beta, \varphi)$ defined as $[23,24]$

$$
x / \cos \varphi=y / \sin \varphi=\frac{c \sin \beta}{\cosh \zeta-\cos \beta}, \quad z=\frac{\mathrm{c} \sinh \zeta}{\cosh \zeta-\cos \beta},
$$

where $\mathbf{c}=\sqrt{\epsilon^{-2}-1}$ is half the (dimensionless) focal distance and

$$
-\infty<\zeta<\infty, \quad 0 \leq \beta \leq \pi, \quad 0 \leq \varphi<2 \pi
$$

The coordinates have the following scale factors:

$$
h_{\zeta}=h_{\beta}=\frac{\mathrm{c}}{\cosh \zeta-\cos \beta}, \quad h_{\varphi}=\rho=\frac{\mathrm{c} \sin \beta}{\cosh \zeta-\cos \beta} .
$$

Figure 2.2 depicts the surfaces of constant $\zeta$ and $\beta$ that are non-intersecting spheres surrounding the foci (points located at $z= \pm \mathrm{c}$ ) and intersecting tori passing through the focal points, respectively. In this curvilinear coordinate system, the thermally

active spheres are represented by $\zeta= \pm \zeta_{0}=\cosh ^{-1} \epsilon^{-1}$ and $(\zeta, \beta) \rightarrow(0,0)$ correspond to $\tilde{r} \rightarrow \infty$.

The boundary-value problem introduced at the beginning of this section can be written in terms of $(\zeta, \beta, \varphi)$ as $[23,24]$ 

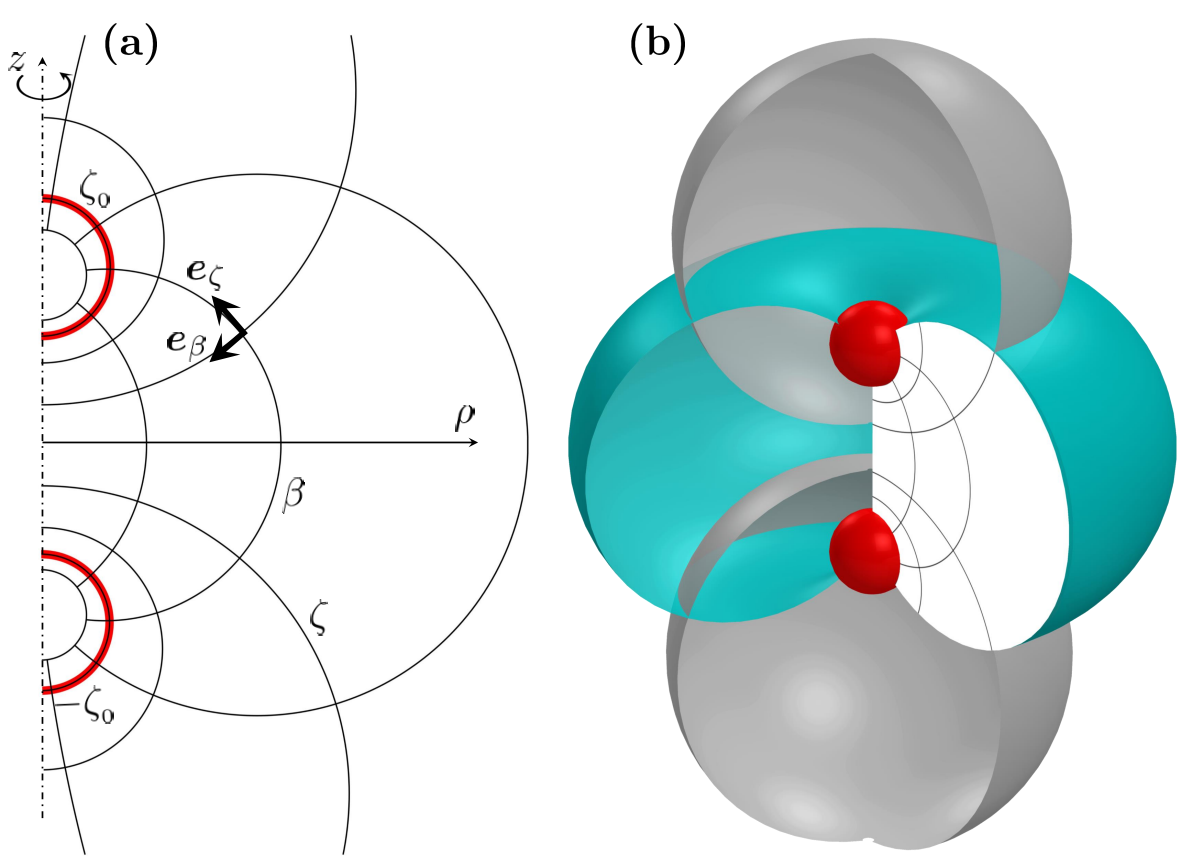

Figure 2.2: (a) Bispherical coordinates in a meridian plane. The arrows show the direction of the unit vectors $\boldsymbol{e}_{\zeta}$ and $\boldsymbol{e}_{\beta}$. (b) Surfaces of constant $\zeta$ and $\beta$ depicted in cyan and gray, respectively. The red curves in (a) and their corresponding surfaces in (b) represent a pair of identical hot/cold spheres that release/absorb heat at a constant uniform rate.

$$
\begin{aligned}
& \nabla^{2} T=\frac{(\cosh \zeta-\cos \beta)^{3}}{c^{2} \sin \beta}\left[\frac{\partial}{\partial \zeta}\left(\frac{\sin \beta}{\cosh \zeta-\cos \beta} \frac{\partial T}{\partial \zeta}\right)\right. \\
&\left.+\frac{\partial}{\partial \beta}\left(\frac{\sin \beta}{\cosh \zeta-\cos \beta} \frac{\partial T}{\partial \beta}\right)+\frac{1}{\sin \beta(\cosh \zeta-\cos \beta)} \frac{\partial^{2} T}{\partial \varphi^{2}}\right]=0 \\
& \text { with }\left.\quad \frac{1}{h_{\zeta}} \frac{\partial T}{\partial \zeta}\right|_{\zeta= \pm \zeta_{0}}= \pm 1 \text { and } T \rightarrow 0 \text { as }(\zeta, \beta) \rightarrow(0,0)
\end{aligned}
$$

The boundary conditions are, again, independent of $\varphi$, which simplifies the Laplace equation to

$$
\frac{\partial}{\partial \zeta}\left(\frac{\sin \beta}{\cosh \zeta-\cos \beta} \frac{\partial T}{\partial \zeta}\right)+\frac{\partial}{\partial \beta}\left(\frac{\sin \beta}{\cosh \zeta-\cos \beta} \frac{\partial T}{\partial \beta}\right)=0
$$

Unlike Eq. (2.8), this equation is not simply separable and belongs to a class of partial differential equations called $R$-separable equations [24]. Therefore, to solve Eq. (2.24), 
we start with the ansatz $T(\zeta, \beta)=\sqrt{\cosh \zeta-\cos \beta} \mathscr{Z}(\zeta) \mathscr{B}(\beta)$, where $\mathscr{Z}$ and $\mathscr{B}$ are the unknown functions we seek to determine. Substituting the proposed form for $T$ into Eq. (2.24), we arrive at a pair of ordinary differential equations for $\mathscr{Z}$ and $\mathscr{B}$. Upon solving those equations, the following general solution for $T$ emerges [23, 24]:

$$
\begin{aligned}
T & =\sqrt{\cosh \zeta-\cos \beta} \sum_{m=0}^{\infty}\left[B_{m}^{1} \sinh (m+1 / 2) \zeta\right. \\
& \left.+B_{m}^{2} \cosh (m+1 / 2) \zeta\right]\left[B_{m}^{3} P_{m}(\cos \beta)+B_{m}^{4} Q_{m}(\cos \beta)\right],
\end{aligned}
$$

where $B_{m}^{1}, \ldots, B_{m}^{4}$ are constant coefficients.

As indicated before, the function $Q_{m}(\cos \beta)$ is singular at $\cos \beta= \pm 1$. Thus, $B_{m}^{4}$ vanish in order to retain the regularity of the solution. One can infer from the geometry of the problem and the boundary conditions that the temperature distribution is symmetric about the $x-y$ plane, which means $\partial T / \partial \zeta=0$ at $\zeta=0$. To satisfy this condition, we must set $B_{m}^{1}=0$. Conveniently, the infinity boundary condition is already satisfied thanks to the term outside the summation that approaches zero as $(\zeta, \beta) \rightarrow(0,0)$. Incorporating these results, the solution for $T$ reduces to

$$
T=\sqrt{\cosh \zeta-\cos \beta} \sum_{m=0}^{\infty} B_{m} \cosh (m+1 / 2) \zeta P_{m}(\cos \beta),
$$

where the constant coefficients $B_{m}$ are determined by enforcing the normal gradient boundary condition on the surface of the spheres, i.e.,

$$
\begin{aligned}
& \left.\frac{1}{h_{\zeta}} \frac{\partial T}{\partial \zeta}\right|_{\zeta=\zeta_{0}}=-\left.\frac{1}{h_{\zeta}} \frac{\partial T}{\partial \zeta}\right|_{\zeta=-\zeta_{0}}=\frac{\sqrt{\cosh \zeta_{0}-\cos \beta}}{c} \\
& \times \sum_{m=0}^{\infty} B_{m}\left[D_{m} P_{m}(\cos \beta)-E_{m} \cos \beta P_{m}(\cos \beta)\right]=1,
\end{aligned}
$$


where

$$
\begin{aligned}
D_{m} & =\frac{\sinh \zeta_{0} \cosh (m+1 / 2) \zeta_{0}}{2} \\
& +(m+1 / 2) \cosh \zeta_{0} \sinh (m+1 / 2) \zeta_{0}, \\
E_{m} & =(m+1 / 2) \sinh (m+1 / 2) \zeta_{0} .
\end{aligned}
$$

Yet, again, we see that the application of the constant flux boundary condition results in a more convoluted equation for the unknown coefficients compared to the situation where the isothermal condition $T=1$ is imposed at $\zeta= \pm \zeta_{0}$ (see, e.g., [29]).

In addition to the orthogonality property Eq. (2.14), the following integral relations apply to the eigenfunction $P_{m}[27]$ :

$$
\begin{aligned}
& \int_{-1}^{1} P_{m}(\mathfrak{x}) P_{n}(\mathfrak{x}) \mathfrak{x} \mathrm{d} \mathfrak{x}=\left\{\begin{array}{ccc}
\frac{2(m+1)}{(2 m+1)(2 m+3)} & \text { if } \quad n=m+1 \\
\frac{2 m}{(2 m-1)(2 m+1)} & \text { if } & n=m-1 \\
0 & \text { if } & n \neq m \pm 1
\end{array}\right. \\
& \int_{-1}^{1} \frac{P_{m}(\mathfrak{x})}{\sqrt{\cosh \zeta_{0}+\mathfrak{x}}} \mathrm{d} \mathfrak{x}=\frac{2 \sqrt{2}}{2 m+1} e^{-(m+1 / 2) \zeta_{0}}
\end{aligned}
$$

Utilizing the above relations in conjunction with Eq. (2.14), we obtain a recursive formula for the coefficients in the form of

$$
\begin{aligned}
B_{1} & =F_{0}+B_{0}, \\
B_{m+1} & =F_{m}+G_{m} B_{m}+\left(1-G_{m}\right) B_{m-1},
\end{aligned}
$$

where

$$
F_{m}=-\frac{2 \sqrt{2} \sinh \zeta_{0} e^{-(m+1 / 2) \zeta_{0}}}{(m+1) \sinh (m+3 / 2) \zeta_{0}}
$$




$$
G_{m}=\frac{1}{m+1}\left[1+\frac{2 m \cosh \zeta_{0} \sinh (m+1 / 2) \zeta_{0}}{\sinh (m+3 / 2) \zeta_{0}}\right] .
$$

We are now left with the task of determining $B_{0}$, accomplished by demanding the terms in the solution that correspond to large eigenvalues to be finite. This condition is satisfied by requiring $B_{m} \rightarrow 0$ as $m \rightarrow \infty$, which, after some mathematical manipulations, yields

$$
B_{0}=-\lim _{m \rightarrow \infty} H_{m}
$$

where

$$
\begin{aligned}
& H_{0}=F_{0}, \quad H_{1}=F_{1}+G_{1} F_{0}, \\
& H_{m}=F_{m}+G_{m} H_{m-1}+\left(1-G_{m}\right) H_{m-2} .
\end{aligned}
$$

With the coefficients known, the mean $T$ over the surface of each sphere is calculated via

$$
\begin{aligned}
\bar{T} & =\frac{1}{\mathbb{S}_{p}} \int_{S_{p}} T \mathrm{~d} S=\frac{2 \pi c^{2}}{\mathbb{S}_{p}} \int_{0}^{\pi} \frac{T \sin \beta}{\left(\cosh \zeta_{0}-\cos \beta\right)^{2}} \mathrm{~d} \beta \\
& =\frac{\sinh \zeta_{0}}{\sqrt{2}} \sum_{m=0}^{\infty} B_{m}\left[1+e^{-(2 m+1) \zeta_{0}}\right] .
\end{aligned}
$$

\subsection{Results and discussion}

We begin with the results for oblate spheroids. First, we consider the limiting cases of $\varepsilon=1$ (sphere) and $\varepsilon=0$ (disk). The results for the former have been already known to be

$$
T=1 / \tilde{r}, \quad \bar{T}=1, \quad \mathrm{Nu}=2 .
$$


For the latter, however, we find

$$
A_{m}=\left\{\begin{array}{ccc}
\frac{i(2 m+1)}{(m-1)(m+2)}\left\{\frac{m !}{2^{m}[(m / 2) !]^{2}}\right\}^{2} & \text { if } & m \equiv 0(\bmod 2) \\
0 & \text { if } & m \equiv 1(\bmod 2)
\end{array},\right.
$$

which, consistent with [19], results in

$$
\bar{T}=8 / 3 \pi, \quad \mathrm{Nu}=3 \pi / 8 .
$$

Next, we examine other aspect ratios. Quite unexpectedly, our calculations indicate that the series solution for the temperature (see Eq. (2.11)) is very well approximated by its first two non-zero terms for the entire range of $\varepsilon$, i.e.,

$$
\begin{aligned}
T & \approx \sqrt{1+\xi_{0}^{2}}\left\{\frac{\mathbb{S}_{p}}{4 \pi} \cot ^{-1} \xi\right. \\
& +\frac{5}{64}\left[\left(2+3 \xi_{0}^{2}\right)+\left(4+3 \xi_{0}^{2}\right)\left(1-\frac{\mathbb{S}_{p}}{2 \pi}\right)\right] \\
& \left.\times\left[\frac{3 \xi-\left(3 \xi^{2}+1\right) \cot ^{-1} \xi}{3\left(1+\xi_{0}^{2}\right) \xi_{0} \cot ^{-1} \xi_{0}-\left(2+3 \xi_{0}^{2}\right)}\right]\left(3 \eta^{2}-1\right)\right\} .
\end{aligned}
$$

In fact, the maximum percent difference between the results corresponding to $m=$ 3 and $m=19$ happens to be less than $4 \%$. A visually convincing demonstration of this result is provided in Fig. 2.3, where we present side-by-side contour plots of the temperature field (in meridian planes) around an oblate spheroid of aspect ratio $\varepsilon=$ 0.3. As you can see, the approximate results of Fig. 2.3a are barely distinguishable from the (nearly) exact results of Fig. 2.3b.

Equally interesting, we also find that the average Nusselt number for oblate spheroids varies almost linearly with the aspect ratio (see Fig. 2.3c). Therefore, the 

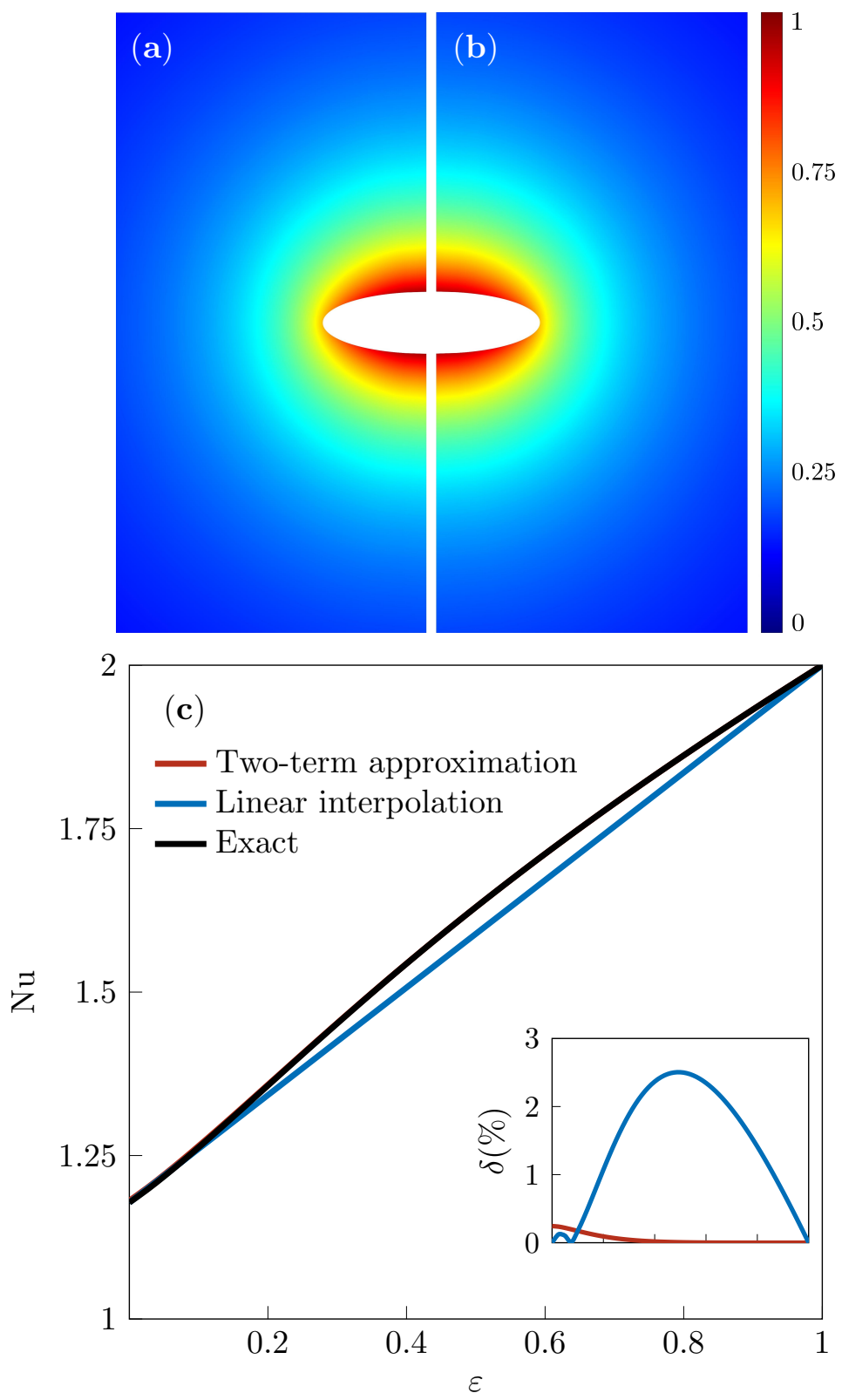

Figure 2.3: (a)-(b) Contour plots of the (dimensionless) temperature field $\theta$ (in meridian planes) around an oblate spheroid of aspect ratio $\varepsilon=0.3$. The left panel illustrates the results of the two-term approximation whereas the right panel presents those obtained by setting $m=19$, considered here as exact results. (c) The Nusselt number $\mathrm{Nu}$ versus the aspect ratio $\varepsilon$ for oblate spheroids. Red, blue, and black lines represent the results corresponding to $m=3$, linear interpolation based on the $\mathrm{Nu}$ of sphere and disk, and $m=19$, respectively. The inset shows the relative difference between the approximate and exact results. 
$\mathrm{Nu}$ versus $\varepsilon$ curve can be approximated by the line that passes through its end points, i.e.,

$$
\mathrm{Nu} \approx \mathrm{Nu}_{\text {disk }}+\left(\mathrm{Nu}_{\text {sphere }}-\mathrm{Nu}_{\text {disk }}\right) \varepsilon=\frac{3 \pi}{8}+\left(2-\frac{3 \pi}{8}\right) \varepsilon .
$$

The maximum relative error of this approximation occurs at $\varepsilon \approx 0.5$ and falls below $3 \%$, which underscores its validity for all aspect ratios (see the inset of Fig. 2.3c). Furthermore, from Fig. 2.3c and its inset, we learn that the Nusselt number calculated based on the two-term representation of $T$ is extremely accurate, differing less than $0.25 \%$ from the actual values.

We now analyze the results for bispheres. In the limit $\epsilon \rightarrow 0$, the spheres are very far from each other and, therefore, the solution simplifies to Eq. (2.36) for a single sphere. On the other hand, when $\epsilon \rightarrow 1$ (i.e., when the gap between the spheres vanish), Eq. (2.26) for the temperature distribution does not immediately reduce to a simple form. The average surface temperature and Nusselt number in this case are $\bar{T}=1.57721$ and $\mathrm{Nu}=1.26806$.

Again, we observe that setting $m=3$ in the series solution for $T$ [Eq. (2.26)] provides excellent results, accurate to within $5 \%$ of the converged solution for $0<$ $\epsilon<4 / 5$ (see, e.g., Figs. 2.4a and 2.4b). As the gap between the spheres narrows, the error of the four-term approximation increases, which means that more terms are needed to represent the temperature field accurately. For example, the error grows to $10 \%$ as the gap size reduces to $\ell / 4$.

Given the recursive relation defined in Eq. (2.30), what carries the most weight in 

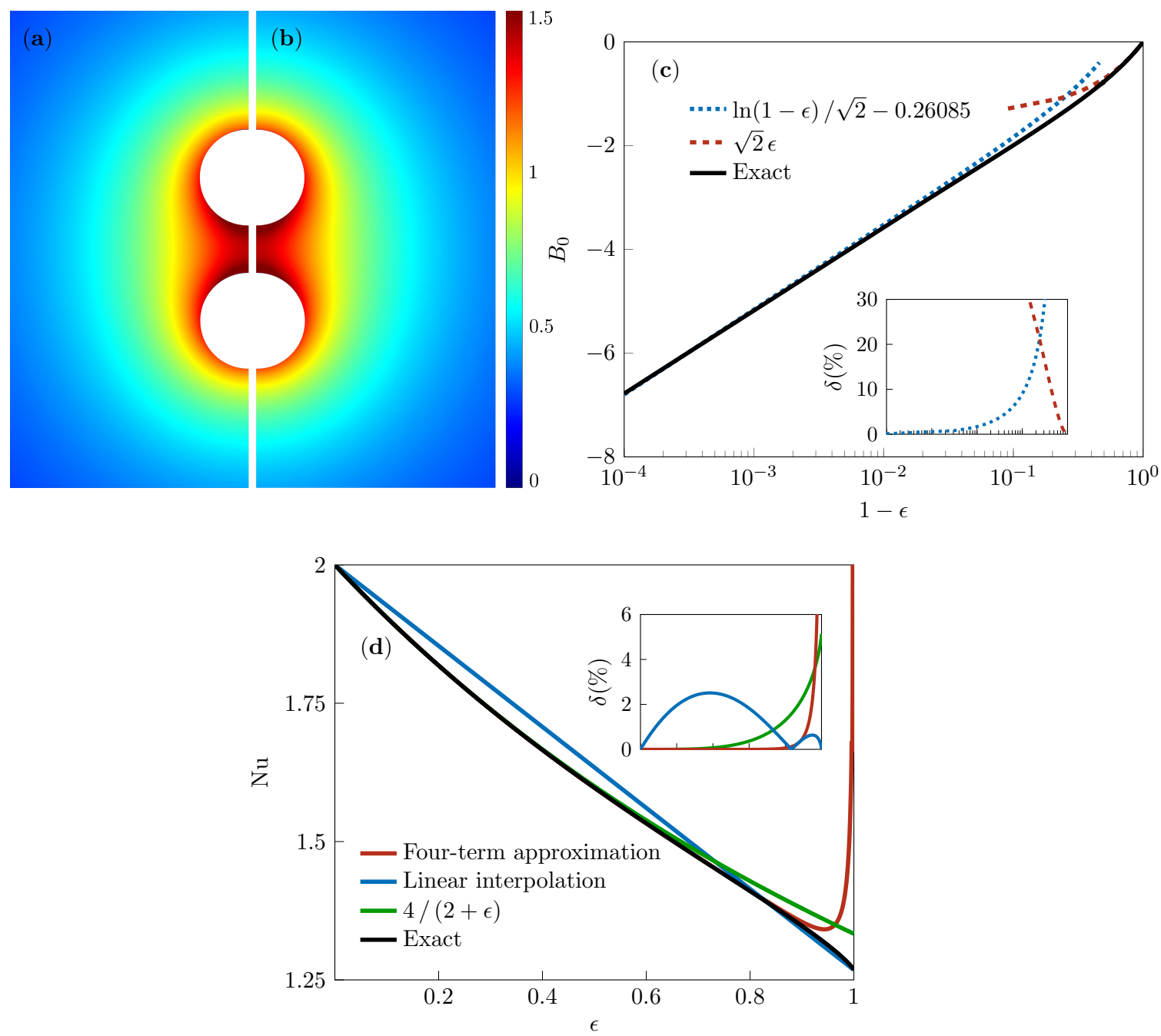

Figure 2.4: (a)-(b) Contour plots of the (dimensionless) temperature field $\theta$ (in meridian planes) around two spheres whose centers are three radii apart $(\epsilon=2 / 3)$. The left panel illustrates the results of the four-term approximation whereas the right panel presents those obtained by setting $m=$ 9 , considered here as exact results. (c) $B_{0}$ as a function of $1-\epsilon$, where the dashed and dotted lines represent the asymptotic approximations in the limits of $\epsilon \rightarrow 0$ and $\epsilon \rightarrow 1$, respectively. And, the inset shows the relative error of the approximations. (d) The Nusselt number $\mathrm{Nu}$ versus the inverse of the dimensionless separation distance $\epsilon$ for a pair of identical spheres. Red, blue, green, and black lines represent the results corresponding to $m=3$, linear interpolation based on the $\mathrm{Nu}$ of single sphere and two touching spheres, asymptotic behavior in the limit of $\epsilon \rightarrow 0$, and the converged solution (i.e., $m \gg 1$ ), respectively. The inset shows the relative difference between the approximate and exact results. 
calculating $B_{m}$ is determining $B_{0}$. Figure $2.4 \mathrm{c}$ shows the variation of this coefficient as a function of $1-\epsilon$. As it can be seen, $B_{0}$ approaches zero as $\sqrt{2} \epsilon$ when the distance between the spheres is large and it asymptotes to $-\infty$ as $\ln (1-\epsilon) / \sqrt{2}-0.26085$ when the spheres almost touch each other. The asymptotic formulas can be used to estimate, with reasonable accuracy, the value of $B_{0}$ over a wide range of $\epsilon$ (see the inset of Fig. 2.4c).

Lastly, our calculations indicate that the Nusselt number of the two-sphere system, like that of the oblate spheroid changes almost linearly with $\epsilon$ (see Fig. 2.4d). Hence, again, a linear interpolation using the $\mathrm{Nu}$ of a single sphere and a pair of touching spheres can be employed to effectively approximate the curve of $\mathrm{Nu}$ versus $\epsilon$ (see the inset of Fig. 2.4d). There exists another approach for approximating this curve based on the asymptotic behavior of $\bar{T}$ in the limit $\epsilon \rightarrow 0$. When the spheres are widely separated, they see each other as a point source/sink. Thus, to the leading order in $\epsilon$, the average surface temperature and consequently the Nusselt number take the forms of $\bar{T}=1+\epsilon / 2$ and $\mathrm{Nu}=4 /(2+\epsilon)$. Perhaps surprisingly, this approximation outperforms the linear interpolation for gap sizes larger than the radius of the spheres (compare blue and green lines in the inset of Fig. 2.4d).

\subsection{Summary}

We derived analytical solutions for the problems of conduction heat transfer from an isolated oblate spheroid and a pair of spheres. The derivations were carried out in 
curvilinear coordinate systems, befitting each geometry, using the method of separation of variables and eigenfunction expansion. While the solutions are in the form of infinite series, we showed that considering only the first few terms provides excellent results, often accurate to within a few percent of the exact values. We also found that the Nusselt number in both cases considered varies rather linearly with respect to the relevant dimensionless length scale of the problem. As we shall see in the following chapters, our results can be used to develop perturbation solutions for the problems of forced convection heat transfer from heated spheroids and bispheres in uniform laminar flows at small Péclet numbers. 


\section{Chapter 3: Forced convection heat trans- fer from a particle at small and large Péclet numbers}

\subsection{Introduction}

The transport of heat from a particle via an externally driven fluid flow is a phenomenon commonly observed in natural and man-made systems. The ubiquity and importance of forced convection heat transfer have led a large number of researchers to study various aspects of this mode of heat transport. Among the investigations conducted to date, many have focused on incompressible laminar flows. Somewhat surprisingly, however, the vast majority of theoretical studies in thhat area have been limited to the case of an object with a known surface temperature distribution (see e.g. $[1,4,6,7,15,30-36])$, while little attention has been paid to the equally practical problem of convection heat transfer from a particle with a prescribed surface heat flux. Of course, in such a problem, the rate of heat transfer (i.e. the surface integral of the imposed heat flux) is already known, but what is not known, and often sought after, is the average surface temperature in response to the heat emanating from the surface of the particle. For example, envisage a scenario where heat is dissipated from 
an electronic element by blowing air over it. Assume that the rate at which the heat is generated by the element is known. In this system, the goal is to set the flow speed such that the average surface temperature stays well below a critical temperature, e.g. the melting temperature of the element.

It is not immediately obvious as to why cases with prescribed surface heat flux condition has been overlooked by theoreticians. However, one might surmise that the inconvenience of applying Neumann, versus Drichlet, boundary conditions is the factor that has deterred them from considering this category of convection heat transfer problems. To partly address this deficiency in the literature, in this chapter, we examine uniform laminar flows past a single hot/cold particle whose surface is presumed to maintain a constant heat flux. Perturbation theory is used to derive approximate expressions for the Nusselt number $\mathrm{Nu}$ (based on the average surface temperature) in the limits of small and large Péclet numbers Pe. The accuracy of the formulas for the specific case of a spheroidal particle in axisymmetric Stokes flow are tested via comparison with finite-volume numerical simulations.

In the rest of this chapter, we first pose the mathematical problem and describe the asymptotic solutions in the limits of diffusion- and advection-dominated heat transport, respectively. Specific results are discussed next and a short summary is given in the end. 


\subsection{Problem statement}

Consider an unbounded steady laminar flow with a divergence-free velocity $\boldsymbol{u}$ past a stationary particle of arbitrary geometry and characteristic length scale $\ell$ (see Fig. 3.1). Let the undisturbed flow field be $\boldsymbol{U}_{\infty}=U_{\infty} \boldsymbol{e}$, where $U_{\infty}=\left|\boldsymbol{U}_{\infty}\right|$ is a constant and $\boldsymbol{e}$ is a unit vector. Suppose that heat is released/absorbed from the surface of the particle at a constant uniform rate $q_{s}$ and that the temperature vanishes at infinity. Neglecting viscous dissipation and assuming that the fluid properties are constant, the boundary-value problem that governs the steady-state distribution of the dimensionless temperature $T$ outside the particle is

$$
\begin{aligned}
\text { Pe } \boldsymbol{u} \cdot \boldsymbol{\nabla} T & =\nabla^{2} T \text { with } \\
\boldsymbol{n} \cdot \boldsymbol{\nabla} T & =-1 \text { for } \boldsymbol{r} \in S_{p} \text { and } \lim _{r \rightarrow \infty} T=0,
\end{aligned}
$$

where the Péclet number is defined as $\mathrm{Pe}=\rho U_{\infty} c_{p} \ell / k$, with $\rho, c_{p}$, and $k$ being the density, specific heat, and thermal conductivity of the fluid, respectively. Also, $\boldsymbol{n}$ is the unit vector outward normal to the surface of the particle denoted by $S_{p}$ and $\boldsymbol{r}$ is the position vector with magnitude $r=|\boldsymbol{r}|$ (see Fig. 3.1). Here, the temperature,

length, and fluid velocity are non-dimensionalized, respectively, by $q_{s} \ell / k, \ell$, and $U_{\infty}$. We reiterate that the primary novelty of our study is the consideration of a Neumann boundary condition on $S_{p}$ in the energy transport equation (3.1).

We, again, define the average Nusselt number as

$$
\mathrm{Nu}=\frac{\mathbb{S}_{p}}{2 \pi \bar{T}},
$$




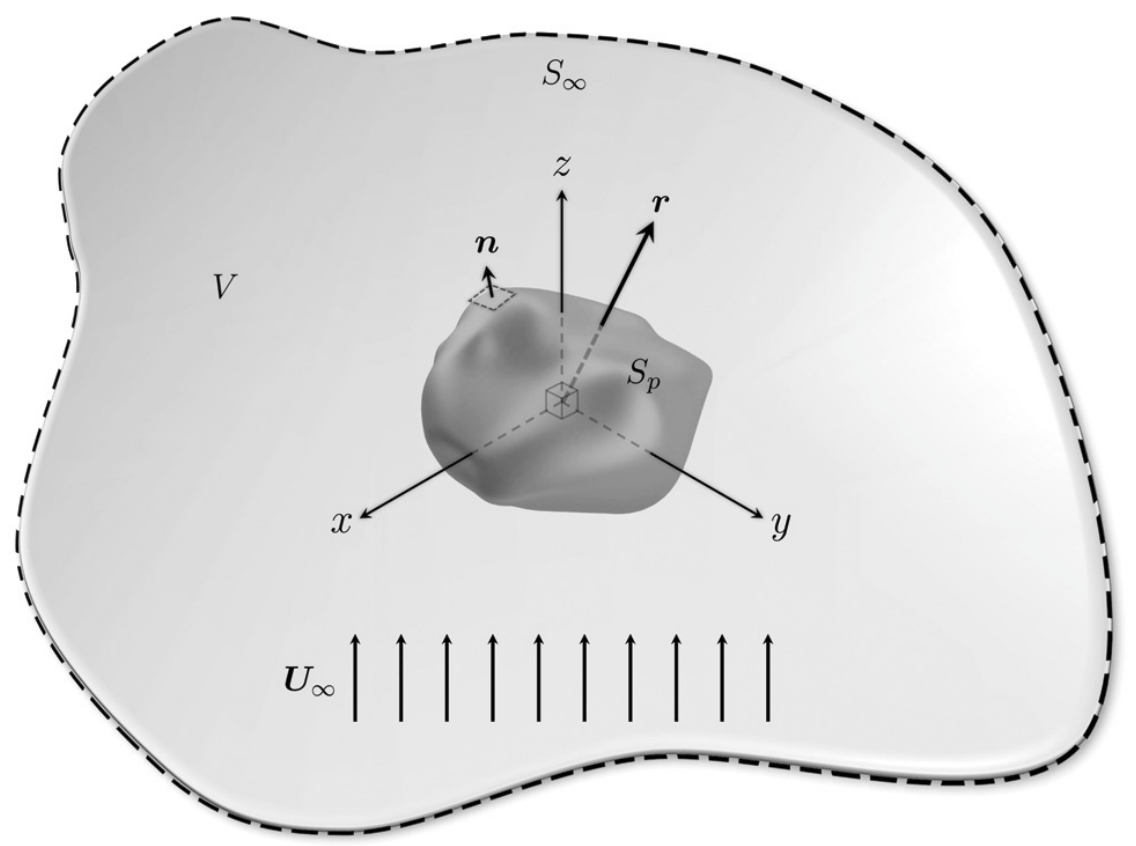

Figure 3.1: Schematic of a stationary particle of arbitrary shape, with surface $S_{p}$ and unit outward normal vector $\boldsymbol{n}$, in a uniform fluid flow. The dashed line indicates an enclosing boundary in the far field.

where $\mathbb{S}_{p}$ represents the dimensionless surface area of the particle and $\bar{T}$ is the mean value of $T$ on $S_{p}$. Our primary objective is to develop approximate formulas for the variation of the Nusselt number (or equivalently $\bar{T}$ ) as a function of the Péclet number. To this end, we use the ideas of the reciprocal theorem in conjunction with the method of matched asymptotic expansions and boundary layer theory to derive expressions that are valid in the limits of $\mathrm{Pe} \ll 1$ and $\mathrm{Pe} \gg 1$. Details of the calculations are described in the following sections. 


\subsection{Perturbation solution in the limit of conduction- dominated heat transport}

Suppose that the Péclet number is small, but finite. In this limit, we seek to determine the $\mathcal{O}(\mathrm{Pe})$ contribution to the Nusselt number. It is well-known that a regular perturbation expansion in terms of Pe is only valid in the vicinity of the particle, i.e.,

regardless of the magnitude of $\mathrm{Pe}$, there exists a domain $\left(r / \ell \gtrsim \mathcal{O}\left(\mathrm{Pe}^{-1}\right)\right)$ where the effect of advection outweighs that of conduction. To remedy this situation, a singular perturbation expansion is used that involves separate expansions covering regions close to and far from the particle, i.e., the inner and outer regions, respectively (see, e.g., $[4,32,34])$. The inner and outer expansions are matched asymptotically in an intermediate region where both expansions are valid and, together, constitute a perturbation solution that is valid in the entire domain.

Specifically, the inner expansion of the temperature field takes the form of

$$
T=T^{(0)}+\operatorname{Pe} T^{(1)}+a(\operatorname{Pe})
$$

which after substitution into Eq. (3.1) leads to

$$
\nabla^{2} T^{(0)}=0 \quad \text { with } \quad \boldsymbol{n} \cdot \boldsymbol{\nabla} T^{(0)}=-1 \text { for } \boldsymbol{r} \in S_{p}
$$

On the other hand, the outer expansion is expressed as

$$
\tilde{T}=\operatorname{Pe} \tilde{T}^{(1)}+a(\mathrm{Pe})
$$


which results in

$$
\boldsymbol{e} \cdot \tilde{\nabla} \tilde{T}^{(1)}=\tilde{\nabla}^{2} \tilde{T}^{(1)} \quad \text { with } \quad \lim _{\tilde{r} \rightarrow \infty} \tilde{T}^{(1)}=0
$$

The $\sim$ overebar in Eqs. (3.5) and (3.6) denotes that the temperature field and the $\boldsymbol{\nabla}$ operator are written in terms of the stretched (rescaled) position vector $\tilde{\boldsymbol{r}}=\operatorname{Pe} \boldsymbol{r}$ with $\tilde{r}=|\tilde{\boldsymbol{r}}|$. The remaining boundary conditions of Eqs. (3.4) and (3.6) are furnished by enforcing

$$
\lim _{r \rightarrow \infty} T=\lim _{\tilde{r} \rightarrow 0} \tilde{T}
$$

at every order of Pe.

We first consider the solution for $T^{(0)}$. To satisfy the matching requirement at the zeroth-order, $T^{(0)} \rightarrow 0$ as $r \rightarrow \infty$, which indicates that $T^{(0)}$ is the conduction $(\mathrm{Pe}=$ 0) solution of the original problem described in Eq. (3.1). Thus, far from the particle, the zeroth-order inner solution can be written as (see, e.g., [4])

$$
T^{(0)}=\frac{\mathbb{S}_{p}}{4 \pi r}+\mathcal{O}\left(r^{-3}\right)=\operatorname{Pe} \frac{\mathbb{S}_{p}}{4 \pi \tilde{r}}+\mathcal{O}\left(\mathrm{Pe}^{-3}\right)
$$

This means that the far-field temperature distribution, to the leading order, may be approximated as the solution of the point source of strength $q_{s} \mathbb{S}_{p} \ell^{2}$ applied at the "heat" center of the particle. Next, given Eq. (3.8) and applying the matching condition again, we find

$$
\tilde{T}^{(1)} \rightarrow \frac{\mathbb{S}_{p}}{4 \pi \tilde{r}} \quad \text { as } \quad \tilde{r} \rightarrow 0
$$

which, together with Eq. (3.6), yields (see, e.g., [4]) 


$$
\tilde{T}^{(1)}=\frac{\mathbb{S}_{p}}{4 \pi \tilde{r}} \exp \left[-\frac{1}{2}(\tilde{r}-\boldsymbol{e} \cdot \tilde{\boldsymbol{r}})\right]
$$

Note that $\tilde{T}^{(1)}$ is also the solution of the point source $q_{s} \mathbb{S}_{p} \ell^{2}$.

We now implement the ideas of the reciprocal theorem (see, e.g., [37, 38]). We multiply the Laplace equation (3.4) by $T$ and Eq. (3.1) by $T^{(0)}$ and then subtract the resulting equations to obtain, after rearranging,

$$
\boldsymbol{\nabla} \cdot\left(T \boldsymbol{\nabla} T^{(0)}\right)=\boldsymbol{\nabla} \cdot\left(T^{(0)} \nabla T\right)-\operatorname{Pe} T^{(0)} \boldsymbol{u} \cdot \boldsymbol{\nabla} T
$$

Integrating this equation over the fluid domain $V$ and using the divergence theorem, we arrive at

$$
\int_{S_{p}} T \boldsymbol{n} \cdot \boldsymbol{\nabla} T^{(0)} \mathrm{d} S=\int_{S_{p}} T^{(0)} \boldsymbol{n} \cdot \nabla T \mathrm{~d} S+\operatorname{Pe} \int_{V} T^{(0)} \boldsymbol{u} \cdot \nabla T \mathrm{~d} V
$$

where the integrands decay sufficiently fast for contributions from surfaces at infinity to vanish. Application of the boundary conditions on $S_{p}$ reduces Eq. (3.12) to

$$
\mathbb{S}_{p} \bar{T}=\int_{S_{p}} T^{(0)} \mathrm{d} S-\mathrm{Pe} \int_{V} T^{(0)} \boldsymbol{u} \cdot \nabla T \mathrm{~d} V=\mathbb{S}_{p}\left(\bar{T}^{(0)}+\mathrm{Pe} \bar{T}^{(1)}\right)+\boldsymbol{a}(\mathrm{Pe})
$$

where $\bar{T}^{(0)}$ is the zeroth-order contribution to the average surface temperatures and $\bar{T}^{(1)}$ is the $\mathcal{O}(\mathrm{Pe})$ correction to it. Given Eqs. (3.13), (3.3), and (3.5), we deduce

$$
-\mathbb{S}_{p} \bar{T}^{(1)}=\int_{V} T^{(0)} \boldsymbol{u} \cdot \boldsymbol{\nabla} T^{(0)} \mathrm{d} V+\boldsymbol{e} \cdot \int_{\mathbb{R}^{3}} \tilde{T}^{(0)} \tilde{\nabla}\left(\tilde{T}^{(1)}-\tilde{T}^{(0)}\right) \mathrm{d} \tilde{\boldsymbol{r}}
$$

where the second integral on the right-hand side is over the entire three-dimensional real space $\mathbb{R}^{3}$ and $\tilde{T}^{(0)}=\mathbb{S}_{p} / 4 \pi \tilde{r}$. The first integral on the right-hand side can be 
written as

$$
\begin{aligned}
& 2 \int_{V} T^{(0)} \boldsymbol{u} \cdot \boldsymbol{\nabla} T^{(0)} \mathrm{d} V=\int_{V} \boldsymbol{\nabla} \cdot\left[\left(T^{(0)}\right)^{2} \boldsymbol{u}\right] \mathrm{d} V \\
& =-\int_{S_{p}}\left(T^{(0)}\right)^{2} \boldsymbol{n} \cdot \boldsymbol{u} \mathrm{d} S-\int_{S_{\infty}}\left(T^{(0)}\right)^{2} \boldsymbol{n} \cdot \boldsymbol{u} \mathrm{d} S
\end{aligned}
$$

where both surface integrals are zero because of the no penetration condition on $S_{p}$ and the incompressibility of the flow (i.e., $\boldsymbol{\nabla} \cdot \boldsymbol{u}=0$ ). Additionally,

$$
\begin{aligned}
& \boldsymbol{e} \cdot \int_{\mathbb{R}^{3}} \tilde{T}^{(0)} \tilde{\nabla} \tilde{T}^{(0)} \mathrm{d} \tilde{\boldsymbol{r}} \\
& =-\left(\frac{\mathbb{S}_{p}}{4 \pi}\right)^{2} \int_{0}^{\infty} \frac{1}{\tilde{r}} \int_{0}^{\pi} \sin ^{2} \theta \int_{0}^{2 \pi} \cos \varphi \mathrm{d} \varphi \mathrm{d} \theta \mathrm{d} \tilde{r}=0
\end{aligned}
$$

Hence, utilizing integration by parts, we find

$$
\bar{T}^{(1)}=-\frac{\mathbb{S}_{p}}{16 \pi^{2}} \int_{\mathbb{R}^{3}} \frac{\boldsymbol{e} \cdot \boldsymbol{r}}{\tilde{r}^{4}} \exp \left[-\frac{1}{2}(\tilde{r}-\boldsymbol{e} \cdot \tilde{\boldsymbol{r}})\right] \mathrm{d} \tilde{\boldsymbol{r}}
$$

Let $\boldsymbol{e}_{x}, \boldsymbol{e}_{y}$, and $\boldsymbol{e}_{z}$ be the unit vectors of the Cartesian coordinate system $(x, y, z)$ located at the center of the particle, see Fig. 3.1. Then, expressing the position vector in spherical coordinates $(\tilde{r}, \theta, \varphi)$ as

$$
\tilde{\boldsymbol{r}}=\tilde{r} \sin \theta \cos \varphi \boldsymbol{e}_{x}+\tilde{r} \sin \theta \sin \varphi \boldsymbol{e}_{y}+\tilde{r} \cos \theta \boldsymbol{e}_{z}
$$

and setting the arbitrary unit vector $\boldsymbol{e}$ to $\boldsymbol{e}_{z}$ (for convenience), the above relation simplifies to

$$
\begin{aligned}
\bar{T}^{(1)}=-\frac{\mathbb{S}_{p}}{16 \pi^{2}} & \int_{0}^{\infty} \int_{0}^{\pi} \int_{0}^{2 \pi} \frac{\cos \theta \sin \theta}{\tilde{r}} \\
& \times \exp \left[-\frac{\tilde{r}}{2}(1-\cos \theta)\right] \mathrm{d} \varphi \mathrm{d} \theta \mathrm{d} \tilde{r}=-\frac{\mathbb{S}_{p}}{8 \pi}
\end{aligned}
$$


which, in turn, yields

$$
\bar{T}=\bar{T}^{(0)}\left(1-\mathrm{Pe} \frac{\mathbb{S}_{p}}{8 \pi \bar{T}^{(0)}}\right)+a(\mathrm{Pe})=\bar{T}^{(0)}\left(1-\mathrm{Pe} \frac{\mathrm{Nu}^{(0)}}{4}\right)+a(\mathrm{Pe})
$$

Substitution of Eq. (3.19) into Eq. (3.2) gives the final expression for the Nusselt number:

$$
\mathrm{Nu}=\frac{\mathbb{S}_{p}}{2 \pi \bar{T}}=\mathrm{Nu}^{(0)}+\mathrm{Pe} \frac{\left(\mathrm{Nu}^{(0)}\right)^{2}}{4}+a(\mathrm{Pe})
$$

Of course, this result is consistent with the one obtained by Leal [34] for the special case of a spherical particle in a uniform Stokes flow.

There are a couple of important points to make here. First, thanks to the reciprocal theorem-inspired approach that was adopted, the derivation of Eqs. (3.19) and (3.20) did not require a detailed knowledge of the velocity field. All we utilized were the facts that the flow is divergence-free and that it does not penetrate into the particle. Even no-slip condition was not essential and we assumed no restriction on the flow Reynolds number defined as $\operatorname{Re}=\rho U_{\infty} \ell / \mu$, where $\mu$ is the fluid viscosity. Second, and perhaps equally notable, Eq. (3.20) for the dependence of the Nusselt number on the Péclet number is identical to the formula obtained by Brenner [4] for an isothermal particle. Equation (3.20) is also the same as the expression derived by Gupalo et al. [39] for the Sherwood number (analogue of $\mathrm{Nu}$ for mass transfer problems) as a function of $\mathrm{Pe}$ for a particle with a first-order chemical reaction occurring on its surface. A natural question to ask at this point is that over what range of Pe does Eq. (3.20) produce accurate results? We will answer this question for the special case 
of axisymmetric Stokes flow past a spheroids in $\S 2.3$.

\subsection{Perturbation solution in the limit of advection- dominated heat transport}

Suppose that the Péclet number is large (i.e., Pe $\gg 1$ ) and the Reynolds number is small or moderate. In this limit, the temperature distribution outside the particle is mainly restricted to a thin layer around the particle (see, e.g., Fig. 3.2), whose thickness is proportional to $\mathrm{Pe}^{-1 / 3}$ (see e.g. [6]). This scaling can be deduced by equating the order of magnitude of the advective and conductive terms in Eq. (3.1), while assuming a linear velocity profile next to $S_{p}$. The restriction on Re is to ensure that, unlike the temperature field, the velocity field surrounding the particle is not confined to a narrow region. Similar to the previous section, here also we wish to develop a two-term asymptotic approximation for the Nusselt number.

Following the standard boundary layer theory (see, e.g., [6]), we assume that the already known velocity field and the to-be-solved-for temperature distribution are axisymmetric or two-dimensional and that $S_{p}$ is smooth. We also adopt the so-called boundary layer coordinate system $(\mathrm{x}, \mathrm{y}, \varphi)$, where the first two components measure, respectively, the distance along and perpendicular to the surface of the particle in the plane of flow (see Fig. 3.2) and the third component is the azimuthal angle for axisymmetric and the distance from the $\mathrm{x}-\mathrm{y}$ plane for two-dimensional problems. The metric coefficients associated with $(\mathrm{x}, \mathrm{y}, \varphi)$ are 


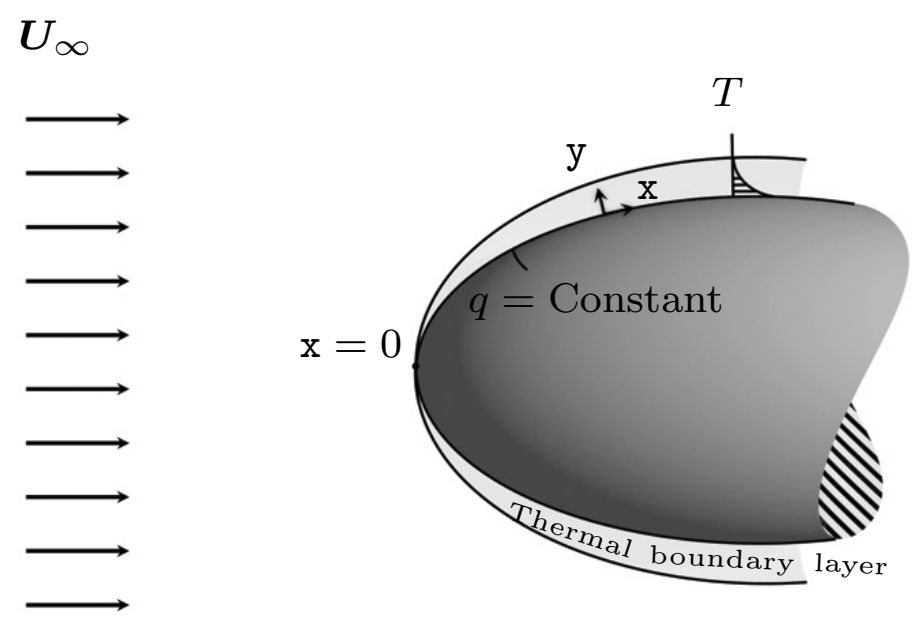

Figure 3.2: Schematic of uniform flow past an axisymmetric heated object at high Péclet number. The boundary layer coordinates are represented by $\mathrm{x}$ and $\mathrm{y}$, and the surface heat flux is denoted by $q$.

$$
h_{\mathrm{x}}=1+\kappa(\mathrm{x}) \mathrm{y}, \quad h_{\mathrm{y}}=1, \quad h_{\varphi}=\varrho(\mathrm{x})+\alpha(\mathrm{x}) \mathrm{y},
$$

where $\kappa$ is the curvature of $S_{p}$. For $2 \mathrm{D}$ cases $\varrho=1$ and $\alpha=0$, whereas for 3D axisymmetric problems $\varrho$ is the rotation radius of $S_{p}$ and $\alpha= \pm \sqrt{1-(d \varrho / d \mathbf{x})^{2}}$ is the cosine of the angle between the axis of rotation and the tangent to $S_{p}$. Thus, $\kappa=$ $-\left(d^{2} \varrho / d \mathrm{x}^{2}\right) / \alpha$ for axisymmetric cases.

In the vicinity of $S_{p}$, Eq. (3.1) can be expressed in terms of the boundary layer coordinates as

$$
\begin{aligned}
& \operatorname{Pe}\left(\frac{u_{\mathrm{x}}}{h_{\mathrm{x}}} \frac{\partial T}{\partial \mathrm{x}}+\frac{u_{\mathrm{y}}}{h_{\mathrm{y}}} \frac{\partial T}{\partial \mathrm{y}}\right) \\
& =\frac{1}{h_{\mathrm{x}} h_{\mathrm{y}} h_{\varphi}}\left[\frac{\partial}{\partial \mathrm{x}}\left(\frac{h_{\mathrm{y}} h_{\varphi}}{h_{\mathrm{x}}} \frac{\partial T}{\partial \mathrm{x}}\right)+\frac{\partial}{\partial \mathrm{y}}\left(\frac{h_{\mathrm{x}} h_{\varphi}}{h_{\mathrm{y}}} \frac{\partial T}{\partial \mathrm{y}}\right)\right] \text { with } \\
& \left.\frac{\partial T}{\partial \mathrm{y}}\right|_{\mathrm{y}=0}=-1 \quad \text { and } \quad \lim _{\mathrm{y} \rightarrow \infty} T=0,
\end{aligned}
$$

where 


$$
u_{\mathrm{x}}=\frac{1}{h_{\varphi} h_{\mathrm{y}}} \frac{\partial \psi}{\partial \mathrm{y}} \quad \text { and } \quad u_{\mathrm{y}}=-\frac{1}{h_{\varphi} h_{\mathrm{x}}} \frac{\partial \psi}{\partial \mathrm{x}}
$$

are the velocity components in the $\mathrm{x}$ and $\mathrm{y}$ directions, respectively, with $\psi$ being the stream function. Remember that $\partial T / \partial \varphi$ and $u_{\varphi}$ are zero. Since we are interested in the solution near $S_{p}$, it is useful to expand $h_{\varphi} / h_{\mathrm{x}}$ and $\psi$ about $\mathrm{y}=0$ as

$$
\begin{aligned}
h_{\varphi} / h_{\mathrm{x}} & =\varrho+(\alpha-\varrho \kappa) \mathrm{y}+\left(\varrho \kappa^{2}-\alpha \kappa\right) \mathrm{y}^{2}+\cdots, \\
\psi & =\psi_{2}(\mathrm{x}) \mathrm{y}^{2}+\psi_{3}(\mathrm{x}) \mathrm{y}^{3}+\cdots,
\end{aligned}
$$

where

$$
2 \psi_{2}=\left.\varrho \frac{\partial u_{\mathrm{x}}}{\partial \mathrm{y}}\right|_{\mathrm{y}=0}=\varrho \tau_{0}
$$

with $\tau_{0}$ being the dimensionless shear stress at the surface of the particle. Note that both $\psi$ and $\partial \psi / \partial \mathrm{y}$ are zero at $\mathrm{y}=0$ due to the no slip condition.

Given how the thickness of the boundary layer scales with the Péclet number, we consider

$$
\tilde{y}=P e^{1 / 3} y
$$

as the stretched coordinate and proceed with rewriting Eq. (3.22) as

$$
\begin{aligned}
& {\left[\mathscr{L}^{(0)}+\mathrm{Pe}^{-1 / 3} \mathscr{L}^{(1)}+\mathcal{O}\left(\mathrm{Pe}^{-2 / 3}\right)\right] T=0 \quad \text { with }} \\
& \left.\frac{\partial T}{\partial \tilde{\mathrm{y}}}\right|_{\tilde{\mathrm{y}}=0}=-\mathrm{Pe}^{-1 / 3} \text { and } \lim _{\tilde{\mathrm{y}} \rightarrow \infty} T=0,
\end{aligned}
$$

where

$$
\mathscr{L}^{(0)}=2 \tilde{\mathrm{y}} \psi_{2} \frac{\partial}{\partial \mathrm{x}}-\tilde{\mathrm{y}}^{2} \frac{d \psi_{2}}{d \mathrm{x}} \frac{\partial}{\partial \tilde{\mathrm{y}}}-\varrho \frac{\partial^{2}}{\partial \tilde{\mathrm{y}}^{2}},
$$




$$
\mathscr{L}^{(1)}=3 \tilde{\mathrm{y}}^{2} \psi_{3} \frac{\partial}{\partial \mathrm{x}}-\tilde{\mathrm{y}}^{3} \frac{d \psi_{3}}{d \mathrm{x}} \frac{\partial}{\partial \tilde{\mathrm{y}}}-(\alpha+\varrho \kappa) \frac{\partial}{\partial \tilde{\mathrm{y}}}\left(\tilde{\mathrm{y}} \frac{\partial}{\partial \tilde{\mathrm{y}}}\right)
$$

Taking the form of Eq. (4.38) into account, it is natural to expand the temperature field, its mean value on $S_{p}$, and the Nusselt number as

$$
\begin{aligned}
T & =\mathrm{Pe}^{-1 / 3}\left(T^{(0)}+\mathrm{Pe}^{-1 / 3} T^{(1)}\right)+\mathcal{O}\left(\mathrm{Pe}^{-1}\right), \\
\bar{T} & =\mathrm{Pe}^{-1 / 3}\left(\bar{T}^{(0)}+\mathrm{Pe}^{-1 / 3} \bar{T}^{(1)}\right)+\mathcal{O}\left(\mathrm{Pe}^{-1}\right), \\
\mathrm{Nu} & =\mathrm{Pe}^{1 / 3} \mathrm{Nu}^{(0)}+\mathrm{Nu}^{(1)}+\mathcal{O}\left(\mathrm{Pe}^{-1 / 3}\right),
\end{aligned}
$$

where

$$
\begin{aligned}
\bar{T} & =\left.\left(\int_{0}^{\mathrm{x}_{m}} \varrho \mathrm{d} \mathrm{x}\right)^{-1} \int_{0}^{\mathrm{x}_{m}} T\right|_{\tilde{\mathrm{y}}=0} \varrho \mathrm{dx}, \\
\mathrm{Nu}^{(0)} & =\frac{\mathbb{S}_{p}}{2 \pi \bar{T}^{(0)}}, \\
\mathrm{Nu}^{(1)} & =-\left(\mathrm{Nu}^{(0)}\right)^{2} \frac{2 \pi \bar{T}^{(1)}}{\mathbb{S}_{p}},
\end{aligned}
$$

with $\mathrm{x}=0$ and $\mathrm{x}_{m}$ corresponding to the forward stagnation point of $S_{p}$ and the maximum value of $\mathrm{x}$, respectively. We note that, for two dimensional problems, the quantities described by Eqs. (3.28) and (3.29) belong to the temperature boundary layer that forms over one side of the stagnation point (see Fig. 3.2), as the energy equation in the two layers can be treated separately, but in the same manner. Furthermore, as clearly articulated by Acrivos and Goddard [6], the above expansions are not expected to be fully applicable to all scenarios, such as where there exists a rear stagnation point on $S_{p}$. Thus, caution needs to be exercised when employing 
these expansions to insure the accuracy of the results.

Below, we derive $\bar{T}^{(0)}$ and $\bar{T}^{(1)}$, and, by extension, $\mathrm{Nu}^{(0)}$ and $\mathrm{Nu}^{(1)}$. Substituting for $T$ in Eq. (3.27) and requiring the energy equation and its boundary conditions to hold for all orders of Pe, we arrive at the following parabolic equations for $T^{(0)}$ and $T^{(1)}$ :

$$
\begin{aligned}
& \mathscr{L}^{(0)} T^{(0)}=0 \quad \text { with } \\
& \left.\frac{\partial T^{(0)}}{\partial \tilde{y}}\right|_{\tilde{y}=0}=-1, \quad \lim _{\tilde{y} \rightarrow \infty} T^{(0)}=0, \quad \text { and } \quad \lim _{\mathrm{x} \rightarrow 0} T^{(0)}=0, \\
& \mathscr{L}^{(0)} T^{(1)}=-\mathscr{L}^{(1)} T^{(0)} \quad \text { with } \\
& \left.\frac{\partial T^{(1)}}{\partial \tilde{y}}\right|_{\tilde{y}=0}=0, \quad \lim _{\tilde{\mathrm{y}} \rightarrow \infty} T^{(1)}=0, \quad \text { and } \quad \lim _{\mathrm{x} \rightarrow 0} T^{(1)}=0,
\end{aligned}
$$

We first consider Eq. (3.30a), which is indeed the standard boundary layer approximation of Eq. (3.27). One might be tempted to develop a similarity solution for this equation, as done traditionally when $S_{p}$ is considered isothermal (see, e.g., [40]). However, such an approach would fail here because of the Neumann boundary condition at $\tilde{y}=0$. Instead, we apply a coordinate transformation of the form (see, e.g., [6])

$$
\mathrm{t}=\int_{0}^{\mathrm{x}} \sqrt{2 \psi_{2}(s)} \varrho(s) \mathrm{d} s \quad \text { and } \quad \mathrm{z}=\sqrt{2 \psi_{2}(\mathrm{x})} \tilde{\mathrm{y}}
$$

which turns Eq. (3.30a) into

$$
\begin{aligned}
& 2 \psi_{2} \varrho\left(z \frac{\partial T^{(0)}}{\partial \mathrm{t}}-\frac{\partial^{2} T^{(0)}}{\partial \mathbf{z}^{2}}\right)=0 \quad \text { with } \\
& \left.\frac{\partial T^{(0)}}{\partial \mathbf{z}}\right|_{z=0}=-\frac{1}{\sqrt{2 \psi_{2}}}, \quad \lim _{\mathbf{z} \rightarrow \infty} T^{(0)}=0, \quad \text { and } \quad \lim _{\mathrm{t} \rightarrow 0} T^{(0)}=0 .
\end{aligned}
$$


According to Sutton [41], the solution of the above problem is

$$
T^{(0)}=-\left.\int_{0}^{\mathrm{t}} \frac{\partial T^{(0)}}{\partial z}\right|_{z=0} G(\mathrm{t}, \mathbf{z} ; \hat{\mathrm{t}}, 0) d \hat{\mathrm{t}}
$$

where

$$
\begin{aligned}
& G(\mathrm{t}, \mathrm{z} ; \hat{\mathrm{t}}, \hat{\mathrm{z}})= \\
& \left\{\begin{array}{cc}
\frac{\sqrt{\mathrm{z} \hat{z}}}{3(\mathrm{t}-\hat{\mathrm{t}})} \exp \left(-\frac{\mathrm{z}^{3}+\hat{z}^{3}}{9(\mathrm{t}-\hat{\mathrm{t}})}\right) I_{-1 / 3}\left(\frac{2(\mathrm{z} \hat{z})^{3 / 2}}{9(\mathrm{t}-\hat{\mathrm{t}})}\right) & \text { for } t>\hat{t} \\
0 & \text { for } t \leq \hat{t}
\end{array}\right.
\end{aligned}
$$

is the so-called Green's function that satisfies

$$
\begin{aligned}
& z \frac{\partial G}{\partial \mathrm{t}}-\frac{\partial^{2} G}{\partial \mathbf{z}^{2}}=\delta(\hat{\mathrm{t}}, \hat{\mathbf{z}}) \text { with } \\
& \left.\frac{\partial G}{\partial \mathbf{z}}\right|_{\mathbf{z}=0}=\lim _{\mathrm{z} \rightarrow \infty} G=\lim _{\mathrm{t} \rightarrow \hat{\mathrm{t}}} G=0 .
\end{aligned}
$$

Here, $I_{-1 / 3}$ is the modified Bessel function of the first kind and of order $-1 / 3$ and $\delta$ is the Dirac delta function. Replacing for $G$ and the heat flux distribution in Eq. (3.33), we find

$$
T^{(0)}=\frac{1}{3^{1 / 3} \Gamma(2 / 3)} \int_{0}^{\mathrm{t}}(\mathrm{t}-\hat{\mathrm{t}})^{-2 / 3}\left(2 \hat{\psi}_{2}\right)^{-1 / 2} e^{-\mathrm{z}^{3} / 9(\mathrm{t}-\hat{\mathrm{t}})} \mathrm{d} \hat{\mathrm{t}}
$$

where $\Gamma$ is the gamma function and $\hat{\psi}_{2}=\psi_{2}(\hat{\mathrm{x}})$. Hence, the leading-order contribution to the average surface temperature can be obtained via (see Eqs. (3.28b) and (3.29a))

$$
\bar{T}^{(0)}=\frac{1}{3^{1 / 3} \Gamma(2 / 3) \int_{0}^{\mathrm{x}_{m}} \varrho \mathrm{dx}} \int_{0}^{\mathrm{x}_{m}} \int_{0}^{\mathrm{x}} \frac{\hat{\varrho}}{(\mathrm{t}-\hat{\mathrm{t}})^{2 / 3}} \mathrm{~d} \hat{\mathrm{x}} \varrho \mathrm{dx}
$$

where $\hat{\varrho}=\varrho(\hat{\mathrm{x}})$ and $\hat{\mathrm{t}}=\mathrm{t}(\hat{\mathrm{x}})$. Remember that all needed to calculate $\mathrm{t}(\mathrm{x})$ is the knowledge of the shear stress distribution on $S_{p}$ (see Eq. (4.33)). 
With the Green's function known, the solution of Eq. (3.30b) can be written formally as

$$
T^{(1)}=\int_{0}^{\mathrm{t}} \int_{0}^{\infty} f(\hat{\mathrm{t}}, \hat{\mathbf{z}}) G(\mathrm{t}, \mathbf{z} ; \hat{\mathrm{t}}, \hat{\mathbf{z}}) \mathrm{d} \hat{\mathbf{z}} \mathrm{d} \hat{\mathrm{t}}
$$

where

$$
\begin{aligned}
f(\mathrm{t}, \mathrm{z}) & =-\frac{\mathscr{L}^{(1)} T^{(0)}}{2 \psi_{2} \varrho} \\
& =-\frac{1}{\sqrt{2 \psi_{2}} \varrho}\left[\mathscr{A} \mathrm{z} \frac{\partial^{2} T^{(0)}}{\partial \mathrm{z}^{2}}-\left(\mathscr{B} \mathrm{z}^{3}+\mathscr{C}\right) \frac{\partial T^{(0)}}{\partial \mathrm{z}}\right] \\
\mathscr{A}(\mathrm{x}) & =\frac{3 \psi_{3}}{2 \psi_{2}} \varrho-(\alpha+\varrho \kappa), \\
\mathscr{B}(\mathrm{x}) & =\frac{d}{d \mathrm{x}}\left(\frac{\psi_{3}}{\left(2 \psi_{2}\right)^{3 / 2}}\right), \\
\mathscr{C}(\mathrm{x}) & =\alpha+\varrho \kappa .
\end{aligned}
$$

Substituting for $T^{(0)}$ in Eq. (3.40a), multiplying the result by Eq. (3.34), and carrying out the $\hat{z}$ integration (see, e.g., $[6,27]$ ), we reach, after some simplifications,

$$
\begin{aligned}
T^{(1)}= & -\left(\frac{1}{3^{1 / 3} \Gamma(2 / 3)}\right)^{2} \int_{0}^{\mathrm{x}}(\mathrm{t}-\hat{\mathrm{t}})^{1 / 3} \\
& \times \exp \left(-\frac{\mathrm{z}^{3}}{9(\mathrm{t}-\hat{\mathrm{t}})}\right) \int_{0}^{\hat{\mathrm{x}}} \frac{1}{(\mathrm{t}-\hat{\hat{\mathrm{t}}})^{2}(\hat{\mathrm{t}}-\hat{\hat{\mathrm{t}}})^{2 / 3}} \\
& \times\{(\mathrm{t}-\hat{\mathrm{t}})[3 \hat{\mathscr{A}}+9(\hat{\mathrm{t}}-\hat{\hat{\mathrm{t}}}) \hat{\mathscr{B}}] \\
& \times{ }_{1} F_{1}\left(2 ; \frac{2}{3} ; \frac{\hat{\mathrm{t}}-\hat{\hat{\mathrm{t}}}}{\mathrm{t}-\hat{\hat{\mathrm{t}}}} \frac{\mathrm{z}^{3}}{9(\mathrm{t}-\hat{\mathrm{t}})}\right)+(\mathrm{t}-\hat{\hat{\mathrm{t}}})(\hat{\mathscr{C}}-2 \hat{\mathscr{A}}) \\
& \left.\times{ }_{1} F_{1}\left(1 ; \frac{2}{3} ; \frac{\hat{\mathrm{t}}-\hat{\hat{\mathrm{t}}}}{\mathrm{t}-\hat{\hat{\mathrm{t}}}} \frac{\mathrm{z}^{3}}{9(\mathrm{t}-\hat{\mathrm{t}})}\right)\right\} \hat{\hat{\varrho}} \mathrm{d} \hat{\hat{\mathrm{x}}} \mathrm{d} \hat{\mathrm{x}},
\end{aligned}
$$

where $\hat{\hat{\mathrm{t}}}=\mathrm{t}(\hat{\hat{\mathrm{x}}}), \hat{\mathscr{A}}=\mathscr{A}(\hat{\mathrm{x}}), \hat{\mathscr{B}}=\mathscr{B}(\hat{\mathrm{x}}), \hat{\mathscr{C}}=\mathscr{C}(\hat{\mathrm{x}}), \hat{\hat{\varrho}}=\varrho(\hat{\hat{\mathrm{x}}})$, and ${ }_{1} F_{1}$ is the confluent 
hypergeometric function of the first kind [25]. The first correction to the mean surface temperature then becomes (see Eqs. (3.28b) and (3.29a))

$$
\begin{aligned}
\bar{T}^{(1)}= & -\frac{1}{\int_{0}^{\mathrm{x}_{m}} \varrho \mathrm{dx}}\left(\frac{1}{3^{1 / 3} \Gamma(2 / 3)}\right)^{2} \int_{0}^{\mathrm{x}_{m}} \int_{0}^{\mathrm{x}}(\mathrm{t}-\hat{\mathrm{t}})^{1 / 3} \\
& \times \int_{0}^{\hat{\mathrm{x}}} \frac{1}{(\mathrm{t}-\hat{\hat{\mathrm{t}}})^{2}(\hat{\mathrm{t}}-\hat{\hat{\mathrm{t}}})^{2 / 3}}\{(\mathrm{t}-\hat{\mathrm{t}})[3 \hat{\mathscr{A}}+ \\
& 9(\hat{\mathrm{t}}-\hat{\mathrm{t}}) \hat{\mathscr{B}}]+(\mathrm{t}-\hat{\hat{\mathrm{t}}})(\hat{\mathscr{C}}-2 \hat{\mathscr{A}})\} \hat{\hat{\varrho}} \mathrm{d} \hat{\mathrm{x}} \mathrm{d} \hat{\mathrm{x}} \varrho \mathrm{dx} .
\end{aligned}
$$

\subsection{Specific results for spheroids in axisymmetric Stokes flow}

We choose Stokes (zero Reynolds number) flow to exemplify the general results of the previous two sections because it is representative of many small-scale flows arising in biology, engineering, and material science. And, we choose spheroids because of their practical significance. In what follows, we first present the results for the limits of small and large Péclet numbers and then show the comparison with the full numerical solution of Eq. (3.1). We acknowledge that the choice of Stokes flow does not affect the low Pe analytical result. For the sake of completeness, we also provide the results for spheroids with isothermal surface condition following the works of Brenner [4] and Acrivos and Goddard [6]. 


\subsubsection{Limit of $\mathrm{Pe} \ll 1$}

Consider a spheroid of equatorial radius $\ell$ and aspect ratio (ratio of polar to equatorial radius) $\varepsilon$. As discussed in $\S 3.3$, when the Péclet number is small, the details of the flow field are irrelevant for calculating the Nusselt number to the leading order in Pe. In this limit, all needed is $\mathrm{Nu}$ corresponding to $\mathrm{Pe}=0$. As discussed in $\S 2.2 .1$ (see also [42]), the conduction Nusselt number of a spheroid follows

$$
\mathrm{Nu}^{(0)}=\frac{\left[-\left(1+\xi_{0}^{2}\right)\right]^{3 / 2} \mathbb{S}_{p}^{2}}{8 \pi^{2} \xi_{0}^{2} \sum_{m=0}^{\infty} 16^{m}(4 m+1) \frac{Q_{2 m}\left(i \xi_{0}\right)}{Q_{2 m}^{\prime}\left(i \xi_{0}\right)} \mathscr{D}_{m}^{2}}
$$

where

$$
\begin{aligned}
\mathscr{D}_{m} & =\sum_{n=0}^{m} \frac{\Gamma(m+n+1 / 2)}{\Gamma(2 n+1) \Gamma(2 m-2 n+1) \Gamma(n-m+1 / 2)} \\
& \times \frac{{ }_{2} \mathrm{~F}_{1}\left(-1 / 2, n+1 / 2 ; n+3 / 2 ;-\xi_{0}^{-2}\right)}{2 n+1} \\
\mathbb{S}_{p} & =2 \pi\left(1+\frac{\xi_{0}^{2}}{\sqrt{1+\xi_{0}^{2}}} \operatorname{coth}^{-1} \sqrt{1+\xi_{0}^{2}}\right) \\
\xi_{0} & =\frac{\varepsilon}{\sqrt{1-\varepsilon^{2}}} .
\end{aligned}
$$

Here, $m$ and $n$ are integers, $Q_{2 m}$ are Legendre functions of the second kind [25] with $Q_{2 m}^{\prime}(\mathfrak{x})=d Q_{2 m}(\mathfrak{x}) / d \mathfrak{x}$, and ${ }_{2} \mathrm{~F}_{1}$ is the hypergeometric function [25]. The summation in the denominator of Eq. (3.43) converges very quickly, to the extent that taking only two terms of the series produces results accurate to within $0.25 \%$ of the exact values. Furthermore, the parameter $\xi_{0}$ is real for $\varepsilon \leq 1$ (oblate spheroids) and is imaginary for $\varepsilon>1$ (prolate spheroids). 
It is interesting to contrast Eq. (3.43) with its counterpart for the problem of conduction heat transfer from a spheroid with a uniform surface temperature $T_{s}$, which is (see, e.g., [26, 43])

$$
\mathbb{N}_{u^{(0)}}=-\frac{\int_{S_{p}} \boldsymbol{n} \cdot \boldsymbol{\nabla} T \mathrm{~d} S}{2 \pi \ell T_{s}}=\frac{2 \sqrt{1-\varepsilon^{2}}}{\cos ^{-1} \varepsilon} .
$$

Allowing for imaginary values of the square root and inverse cosine functions, this expression and also the forthcoming Eq. (3.50) are valid for the entire range of $\varepsilon$. Here,

the Nusselt number is denoted by a different symbol so it is easily distinguished from its analog for the constant heat flux surface condition. Clearly, Eq. (3.47) is far less cumbersome than Eq. (3.43). That aside, the substitution of either Eq. (3.43) or (3.47) in Eq. (3.20) gives the Nusselt number correct to the order of Pe for incompressible uniform laminar flows past a spheroid.

\subsubsection{Limit of $\mathrm{Pe} \gg 1$}

Consider the spheroid of $\S 3.5 .1$ with its axis of revolution coinciding with the $z$ axis of the Cartesian coordinate system, and suppose that $\boldsymbol{e}=\boldsymbol{e}_{z}$. To determine the $\mathcal{O}\left(\mathrm{Pe}^{1 / 3}\right)$ Nusselt number and its first correction, we need to calculate the integrals in Eqs. (3.38) and (3.42), which involve the functions $\varrho(\mathrm{x}), \mathrm{t}(\mathrm{x}), \mathscr{A}(\mathrm{x}), \mathscr{B}(\mathrm{x})$, and $\mathscr{C}(\mathrm{x})$. The first and last functions depend only on the geometry of the spheroid whereas the remaining three are additionally dependent on the flow field. It is more convenient to express these functions in the terms of a new variable $\eta$, where 


$$
\frac{d \mathrm{x}}{d \eta}=-h_{\eta}=-\sqrt{\frac{\varepsilon^{2}+\left(1-\varepsilon^{2}\right) \eta^{2}}{1-\eta^{2}}} \quad \text { and } \quad-1 \leq \eta \leq 1
$$

with $\eta=1$ and $\eta=-1$ corresponding to $\mathrm{x}=0$ and $\mathrm{x}=\mathrm{x}_{m}$, respectively. The stream function for axisymmetric Stokes flow past a spheroid is known (see, e.g., [28]). Granted this, we find, following the definitions given in $\S 3.4$, that

$$
\begin{aligned}
\varrho(\eta) & =\sqrt{1-\eta^{2}}, \\
\mathrm{t}(\eta) & =\sqrt{\frac{F \varepsilon}{16 \pi}}\left(\cos ^{-1} \eta-\eta \sqrt{1-\eta^{2}}\right), \\
\mathscr{A}(\eta) & =\frac{1}{2 h_{\eta} \varepsilon}\left[\left(1-\varepsilon^{2}\right)-\frac{5 \varepsilon^{2}}{\eta^{2}+\varepsilon^{2}\left(1-\eta^{2}\right)}\right] \\
\mathscr{B}(\eta) & =\frac{\eta}{3 h_{\eta}} \sqrt{\frac{\pi}{F}}\left[\frac{1}{\left(1-\eta^{2}\right) \varepsilon}\right]^{3 / 2}\left\{\frac{3 \varepsilon^{2}}{\left[\eta^{2}+\varepsilon^{2}\left(1-\eta^{2}\right)\right]^{2}}\right. \\
& \left.\times\left[1+3\left(\varepsilon^{2}-1\right)\left(1-\eta^{2}\right)\right]-\left(1+\varepsilon^{2}\right)\right\} \\
\mathscr{C}(\eta) & =\frac{\varepsilon}{h_{\eta}}\left[1+\frac{1}{\eta^{2}+\varepsilon^{2}\left(1-\eta^{2}\right)}\right],
\end{aligned}
$$

where

$$
F=\frac{8 \pi\left(1-\varepsilon^{2}\right)^{3 / 2}}{\left(1-2 \varepsilon^{2}\right) \cos ^{-1} \varepsilon+\varepsilon \sqrt{1-\varepsilon^{2}}}
$$

is the magnitude of the Stokes drag experienced by the spheroid. This quantity is made dimensionless by $\mu U_{\infty} \ell$. The average surface temperature and its first correction can now be calculated by replacing the foregoing relations in Eqs. (3.38) and (3.42). Substitution of $\bar{T}^{(0)}$ and $\bar{T}^{(1)}$ in Eqs. (3.29b) and (3.29c) then yields $\mathrm{Nu}^{(0)}$ and $\mathrm{Nu}^{(1)}$.

Equations (3.49) can also be used to determine the $\mathcal{O}\left(\mathrm{Pe}^{1 / 3}\right)$ and $\mathcal{O}(1)$ contributions to the Nusselt number for high-Péclet number heat transfer from an isothermal 
spheroid in axisymmetric Stokes flow. Availing ourselves of the general results of Acrivos and Goddard [6], it can be shown, after much reduction, that

$$
\begin{aligned}
& \mathbb{N}_{\Theta^{(0)}}=\frac{1}{8 \Gamma(4 / 3)}(12 \pi F \varepsilon)^{1 / 3}, \\
& \mathbb{N}_{\Theta^{(1)}}=\mathbb{N}_{\text {sphere }}^{(1)} \frac{4 \varepsilon^{2}+1}{5 \varepsilon},
\end{aligned}
$$

where

$$
\begin{aligned}
\mathbb{N} u_{\text {sphere }}^{(1)} & =0.92301=\frac{5}{3}\left\{1-\frac{4 \Gamma(2 / 3)}{[\Gamma(1 / 3)]^{2}} \int_{0}^{\pi}\left(\frac{\gamma-\frac{1}{2} \sin 2 \gamma}{\pi}\right)^{-2 / 3}\right. \\
& \left.\times\left(1-\frac{\gamma-\frac{1}{2} \sin 2 \gamma}{\pi}\right)^{1 / 3} \frac{1-\cos \gamma}{\pi} \sin ^{2} \gamma \mathrm{d} \gamma\right\}
\end{aligned}
$$

Again, simplicity-wise, the contrast between the above formulas and those for $\mathrm{Nu}^{(0)}$ and $\mathrm{Nu}^{(1)}$ is quite remarkable. Equation (3.51a) was also reported by Sehlin [9], though in a different form. However, to the best of our knowledge, Eq. (3.51b) has not been reported elsewhere, and is, therefore, another original contribution of this work.

Lastly, we note that the existence of a rear stagnation point on the spheroid in Stokes flow renders the perturbation expansion described by Eq. (3.28a) invalid in the vicinity of $\eta=-1$. Fortunately, however, the contribution of this singular region to the Nusselt number is beyond $\mathcal{O}(1)$, and, hence, has no effect on $\mathrm{Nu}^{(1)}$ and $\mathbb{N u}^{(1)}$ $($ see $[6])$. 


\subsubsection{Comparison with direct numerical solutions}

To find out the true limits for which the perturbation calculations of $\S 3.5 .1$ and $\S 3.5 .2$ for the Nusselt number are valid, we compare our theoretical results with those obtained from the full numerical solution of Eq. (3.1). A second-order finite volume method as implemented in OpenFOAM (see, e.g., [22]) is used to perform the numerical calculations. The Stokes equations for the velocity field $\boldsymbol{u}$ is solved first using the SIMPLE algorithm, and the advection-diffusion equation for the temperature distribution $T$ is treated next. The outer boundary at infinity is modeled as a large cylinder, whose center coincides with the center of the spheroid. The diameter of the cylinder is equal to its length, which is 200 times the semi-major axis of the spheroid. 2D axisymmetric meshes concentrated around $S_{p}$ are employed to discretize the physical domain and grid-independence tests are performed by refining the mesh in the entire domain and repeating the simulations. In all cases considered, the computational grid is chosen such that the change in the results due to the refinement is marginal.

Figure 3.3 shows the results of the numerical calculations for the spheroids of various aspect ratios. Interestingly, the plots of $\mathrm{Nu}$ versus $\mathrm{Pe}$ for constant heat flux and isothermal boundary conditions are very much alike, not only qualitatively but also quantitatively (compare solid and dashed lines).

Perhaps, the simplest way to construct a global approximation for the Nusselt number based on the two-term asymptotic expressions for $\mathrm{Pe} \ll 1$ and $\mathrm{Pe} \gg 1$ (see Eqs. (3.53) and (3.54)) is 


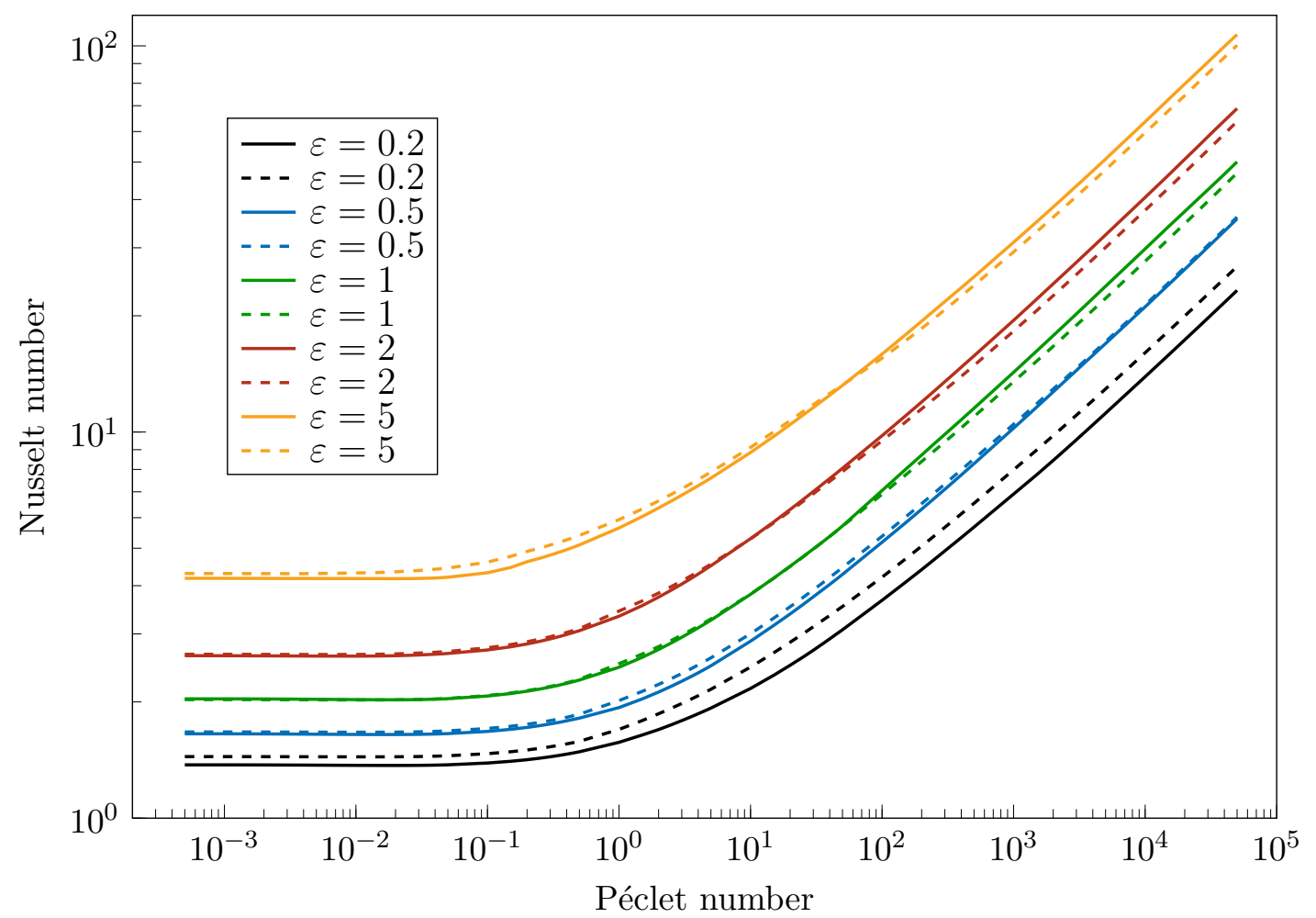

Figure 3.3: Numerically calculated plots of the Nusselt number versus Péclet number for forced convection heat transfer from spheroids of various aspect ratios in an axisymmetric uniform Stokes flow subject to constant heat flux (solid lines) and isothermal (dashed lines) boundary conditions on the surface of the spheroid.

$$
\mathrm{Nu}=\left\{\begin{array}{lll}
\mathrm{Nu}_{l}^{(0)}+\mathrm{Pe} \frac{\left(\mathrm{Nu}_{l}^{(0)}\right)^{2}}{4} \quad \text { if } \quad \mathrm{Pe}<\mathrm{Pe}_{c} \\
\mathrm{Pe}^{1 / 3} \mathrm{Nu}_{h}^{(0)}+\mathrm{Nu}_{h}^{(1)} \quad \text { if } \quad \mathrm{Pe} \geq \mathrm{Pe}_{c}
\end{array},\right.
$$

where $\mathrm{Pe}_{c}$ is the cut-off Péclet number and the subscripts $l$ and $h$ indicate that the coefficients belong to the low and high Pe limits, respectively. Alternatively, the Nusselt number may be approximated over the entire range of the Péclet number by (see $[43,44])$

$$
\mathrm{Nu}=\frac{\mathrm{Nu}_{l}^{(0)}}{2}+\left[\left(\frac{\mathrm{Nu}_{l}^{(0)}}{2}\right)^{3}+\left(\mathrm{Nu}_{h}^{(0)}\right)^{3} \mathrm{Pe}\right]^{1 / 3},
$$


which only incorporates the leading order terms in the perturbation expansions of $\mathrm{Nu}$ in the asymptotic limits of Pe. Figure 3.4 presents the percent difference between the results of Fig. 3.3 and those of Eqs. (3.53) and (3.54). Overall, we see that both approximations are quite accurate. Specifically, Eq. (3.53) provides more precise predictions when $\mathrm{Pe} \ll 1$ and $\mathrm{Pe} \gg 1$ whereas better estimates are given by Eq. (3.54) at intermediate values of $\mathrm{Pe}$, where the approximations deviate the most from the numerical results. Finally, we note that the kinks in Figs. 3.4a and 3.4c are associated with transition from low- to high-Péclet-number solutions at $\mathrm{Pe}_{c}$, which vary from 0.2 to 1 for the range of aspect ratios considered. In comparison with the plots for $\varepsilon=0.2,0.5,1,2$, the transition in the curves corresponding to $\varepsilon=5$ is smoother as, in these cases, both asymptotic formulas overestimate the Nusselt number (i.e., $\Delta$ is positive) in the neighborhood of the cut-off point and no discontinuity exist at $\mathrm{Pe}_{c}$.

\subsection{Summary}

We examined the problem of heat transfer from a stationary hot (or cold) particle immersed in an unbounded fluid in the presence of a uniform background flow. We used the perturbation theory to derive two-term approximations for the average Nusselt number in the asymptotic limits of the Péclet number. At small $\mathrm{Pe}, \mathrm{Nu}$ was approximated as the summation of the conduction $\mathrm{Nu}$ and the $\mathcal{O}(\mathrm{Pe})$ correction. We showed that, for arbitrary particle shapes and flow Reynolds numbers, the correction term is equal to the square of the zeroth-order term divided by four (see Eq. (3.20)). At high 

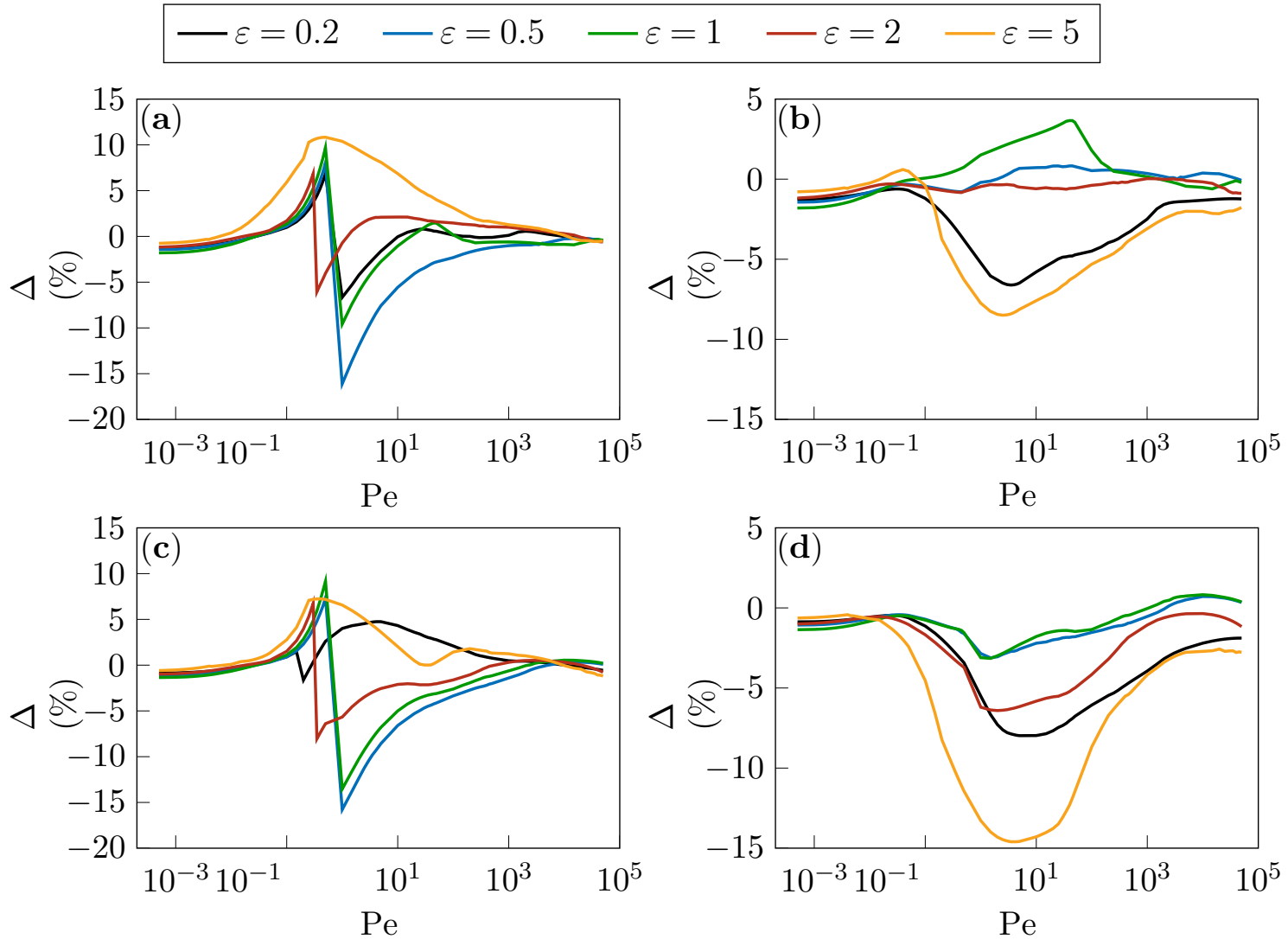

Figure 3.4: Percent difference $\Delta$ between the numerical and approximate asymptotic results for the Nusselt number corresponding to (a)-(b) constant heat flux and (c)-(d) isothermal boundary conditions. (a) $-(\mathrm{c})$ and (b) $-(\mathrm{d})$ present $\Delta$ versus Pe curves for approximations based on Eqs. (3.53) and (3.54), respectively. The cut-off Péclet numbers for the aspect ratios $\varepsilon=$ $0.2,0.5,1,2,5$ in (a) and (c) are, respectively, $\mathrm{Pe}_{c}=0.2,1,1,0.35,0.25$ and $\mathrm{Pe}_{c}=1,1,1,0.35,0.25$.

Pe, the boundary layer theory was employed to analytically solve for the temperature distribution within the thermal boundary layer up to $\mathcal{O}\left(\mathrm{Pe}^{-2 / 3}\right)$. The solutions were used to calculate the $\mathcal{O}\left(\mathrm{Pe}^{1 / 3}\right)$ and $\mathcal{O}(1)$ contributions to the Nusselt number. These calculations were restricted to axisymmetric and two-dimensional problems with low to moderate Reynolds numbers. It is important to note that the primary novelty of both low and high Péclet number results are due to the assumption of constant heat 
flux condition on the surface of the particle.

We exemplified the general perturbation calculations through the problem of axisymmetric Stokes flow past a spheroid. The specific results were then compared against those obtained from the full numerical solution of the underlying conservation of thermal energy equation. The comparisons confirmed the accuracy of the approximations for $\mathrm{Nu}$ over a wide range of $\mathrm{Pe}$. 


\section{Chapter 4: Heat transfer from a particle in laminar flows of a variable ther- mal conductivity fluid}

\subsection{Introduction}

Heat transfer from a hot or cold object exposed to an external fluid flow is arguably the most basic form of forced convection heat transfer encountered in industrial processes and technological applications. When analyzing this category of heat transfer problems, in many cases, it is well justified to assume that the fluid properties such as density $\rho$, viscosity $\mu$, specific heat $c_{p}$, and thermal conductivity $k$ are constant. In general, this assumption renders the energy equation (the partial differential equation that governs the distribution of the fluid temperature) linear and decoupled from the Navier-Stokes equations (from which the fluid velocity field is determined).

There exist, however, practical cases where at least one of the fluid properties cannot realistically be considered constant. For instance, it has been shown that the effective thermal conductivity of nanofluids increases considerably with the rising temperature (see, e.g., [45]) or, for liquid metals, $k$ has been found to vary roughly linearly with the temperature in a wide range of operating conditions (see, e.g., [46]). 
The energy equation in these situations is no longer linear and, therefore, becomes more challenging to solve, which is the cost of adding realism to the mathematical model of the underlying transport phenomenon. Perhaps for this reason, the majority of textbook examples and classical problems in convective heat transfer from objects are solved under the assumption that the fluid properties are constant. Hence, it is of both academic and practical interest to revisit those problems with the goal of extending their solutions to cases with variable fluid properties. To this end, in this chapter, we examine the steady-state transfer of heat from a particle via an externally driven laminar flow, with the premise that the thermal conductivity of the fluid is a linear function of the temperature.

Building on previous theoretical efforts on the subject (see, e.g., [21, 44, 47-58]), we derive approximate expressions for the Nusselt number of particles of arbitrary geometry. The derivations are based on asymptotic and scaling analyses. For completeness, we consider both constant temperature and uniform heat flux boundary conditions on the surface of the particle. The results are presented for the full range of Péclet number Pe.

In the rest of this chapter, we first describe the problem we wish to solve. Then, we present the solutions for the above-mentioned surface conditions. The validity ranges of the solutions are discussed next and a brief summary is given in the end. 


\subsection{Problem statement}

Consider a laminar, steady-state, incompressible flow with velocity $\boldsymbol{u}$ past a stationary object of arbitrary geometry and characteristic length $\ell$. Suppose that the free-stream velocity takes the form of $\boldsymbol{U}_{\infty}=U_{\infty} \boldsymbol{e}$, where $U_{\infty}=\left|\boldsymbol{U}_{\infty}\right|$ is a constant and $\boldsymbol{e}$ is a unit vector, and that the temperature approaches a constant value, denoted $T_{\infty}^{\star}$, at large distances from the particle. Also, let the thermal conductivity of the fluid vary with the dimensionless temperature as $k=k_{0}(1+\beta T)$, with $k_{0}$ and $\beta$ being the farfield conductivity and a constant parameter, respectively. Then, neglecting viscous dissipation, the equation that governs the steady-state distribution of $T$ outside the particle is

$$
\operatorname{Pe} \boldsymbol{u} \cdot \boldsymbol{\nabla} T=\boldsymbol{\nabla} \cdot[(1+\beta T) \boldsymbol{\nabla} T],
$$

where the Péclet number is defined as $\mathrm{Pe}=\rho U_{\infty} c_{p} \ell / k_{0}$. The boundary conditions associated with Eq. (4.1) are

$$
T=1 \quad \text { for } \quad \boldsymbol{r} \in S_{p} \quad \text { and } \quad \lim _{r \rightarrow \infty} T=0,
$$

for the case in which the surface of the particle $S_{p}$ is held at a constant temperature $T_{s}$, and

$$
(1+\beta T)(\boldsymbol{n} \cdot \boldsymbol{\nabla} T)=-1 \quad \text { for } \quad \boldsymbol{r} \in S_{p} \quad \text { and } \quad \lim _{r \rightarrow \infty} T=0
$$

for the one where a uniform heat flux $q_{s}$ is applied on $S_{p}$. As before, $\boldsymbol{r}$ is the position vector with magnitude $r=|\boldsymbol{r}|$ and $\boldsymbol{n}$ is the unit vector outward normal to the surface 
of the particle. Furthermore, the length and fluid velocity are non-dimensionalized, respectively, by $\ell$ and $U_{\infty}$, whereas the dimensionless temperature is defined as either

$$
\frac{T^{\star}-T_{\infty}^{\star}}{T_{s}^{\star}-T_{\infty}^{\star}} \quad \text { or } \quad \frac{T^{\star}-T_{\infty}^{\star}}{q_{s} \ell / k_{0}},
$$

consistent with the boundary conditions in Eqs. (4.2) and (4.3). The star superscript is used to denote the dimensional temperature. Also, the average Nusselt numberis defined as

$$
\mathrm{Nu}_{T}=-\frac{1+\beta}{2 \pi} \int_{S_{p}} \boldsymbol{n} \cdot \boldsymbol{\nabla} T \mathrm{~d} S
$$

for the problem with a prescribed temperature on the surface of the particle (see Eq. $(4.2))$ and as

$$
\mathrm{Nu}_{Q}=\frac{\mathbb{S}_{p}}{2 \pi \bar{T}}
$$

for the one with a prescribed heat flux on $S_{p}$ (see Eq. (4.3)), where $\mathbb{S}_{p}$ represents the dimensionless surface area of the particle and $\bar{T}$ is the mean value of $T$ on $S_{p}$. In the following sections, we seek to develop approximate formulas for the variations of $\mathrm{Nu}_{T}$ and $\mathrm{Nu}_{Q}$ with Pe and $\beta$.

\subsection{Variation of Nusselt number for constant tem- perature boundary condition}

We begin the derivation of $\mathrm{Nu}_{T}$ by first considering the asymptotic limits of small and large Péclet numbers and then bridging the gap between the two limits by introducing 
a smooth transition function. Our approach builds on ideas presented in the classical works on the topic of heat and mass transfer from an isolated particle in uniform flows (see, e.g., $[4,6,21,34,44,49])$.

\subsubsection{Limit of conduction-dominated heat transport}

Suppose conduction is the dominant mode of heat transport, i.e., $\mathrm{Pe} \ll 1$, but finite. In this limit, an effective approach for dealing with the nonlinearity of Eq. (4.1) is to make a change of variable from $T$ to $\theta$ such that

$$
\theta=\frac{1}{k_{0}} \int_{0}^{T} k(\mathscr{T}) \mathrm{d} \mathscr{T}=\int_{0}^{T}(1+\beta \mathscr{T}) \mathrm{d} \mathscr{T}=\frac{\beta}{2} T^{2}+T .
$$

This is known as the Kirchhoff transformation (see, e.g., [21, 52]), with the reference temperature set to $T=0$ for convenience. It follows from Eq. (4.7) that

$$
\mathrm{Nu}_{T}=-\frac{1}{2 \pi} \int_{S_{p}} \boldsymbol{n} \cdot \nabla \theta \mathrm{d} S
$$

where

$$
\begin{aligned}
& \operatorname{Pe} \boldsymbol{u} \cdot \frac{\nabla \theta}{\sqrt{1+2 \beta \theta}}=\nabla^{2} \theta, \text { with } \\
& \theta=1+\beta / 2 \text { for } \boldsymbol{r} \in S_{p} \text { and } \lim _{r \rightarrow \infty} \theta=0 .
\end{aligned}
$$

Our goal is to obtain an asymptotic expression for $\mathrm{Nu}_{T}$, and, to that end, we proceed with a singular perturbation expansion of $\theta$ in terms of Pe (see§3.5.1). Again, we assume that, in the vicinity of the particle, $\theta$ can be expressed as 


$$
\theta=\theta^{(0)}+\operatorname{Pe} \theta^{(1)}+a(\mathrm{Pe})
$$

which is known as the inner expansion of $\theta$. Upon substitution of Eq. (4.10) into Eqs. (4.8) and (4.9), we obtain

$$
\begin{aligned}
& \mathrm{Nu}_{T}= \mathrm{Nu}_{T}^{(0)}+\mathrm{PeNu}_{T}^{(1)}+a(\mathrm{Pe}) \\
&=-\frac{1}{2 \pi}\left[\int_{S_{p}} \boldsymbol{n} \cdot \boldsymbol{\nabla} \theta^{(0)} \mathrm{d} S+\mathrm{Pe} \int_{S_{p}} \boldsymbol{n} \cdot \boldsymbol{\nabla} \theta^{(1)} \mathrm{d} S\right]+\boldsymbol{a}(\mathrm{Pe}) \\
& \nabla^{2} \theta^{(0)}=0 \quad \text { with } \quad \theta^{(0)}=1+\beta / 2 \quad \text { for } \quad \boldsymbol{r} \in S_{p} .
\end{aligned}
$$

Far from the particle, on the other hand, we consider $\theta$ to take the form of

$$
\tilde{\theta}=\operatorname{Pe} \tilde{\theta}^{(1)}+a(\mathrm{Pe})
$$

where the tilde overbar denotes that the transformed temperature field is written as a function of the rescaled position vector

$$
\tilde{\boldsymbol{r}}=\operatorname{Pe} \boldsymbol{r} \quad \text { with } \quad \tilde{r}=|\tilde{\boldsymbol{r}}|
$$

Rewriting Eq. (4.9) in terms of $\tilde{\boldsymbol{r}}$ and replacing Eq. (4.13) for $\tilde{\theta}$, we find

$$
\boldsymbol{e} \cdot \tilde{\nabla} \tilde{\theta}^{(1)}=\tilde{\nabla}^{2} \tilde{\theta}^{(1)} \quad \text { with } \quad \lim _{\tilde{r} \rightarrow \infty} \tilde{\theta}^{(1)}=0
$$

with $\tilde{\nabla}$ and $\tilde{\nabla}^{2}$ operators representing derivatives with respect to the stretched coordinates. The inner and outer expansions are required to match asymptotically, i.e.,

$$
\lim _{r \rightarrow \infty} \theta=\lim _{\tilde{r} \rightarrow 0} \tilde{\theta}
$$


Enforcing the above equation at every order of Pe, furnishes the missing boundary conditions of Eqs. (4.12) and (4.15). Following Brenner [4], the zeroth-order inner solution away from the particle and the first-order outer solution can be written, respectively, as

$$
\begin{aligned}
& \theta^{(0)}=\frac{\mathrm{Nu}_{T}^{(0)}}{2 r}+\mathcal{O}\left(r^{-3}\right)=\operatorname{Pe} \frac{\mathrm{Nu}_{T}^{(0)}}{2 \tilde{r}}+\mathcal{O}\left(\mathrm{Pe}^{-3}\right), \\
& \tilde{\theta}^{(1)}=\frac{\mathrm{Nu}_{T}^{(0)}}{2 \tilde{r}} \exp \left[-\frac{1}{2}(\tilde{r}-\boldsymbol{e} \cdot \tilde{\boldsymbol{r}})\right]
\end{aligned}
$$

where $\boldsymbol{r}$ is measured from a proper origin located at the particle's "heat center".

Applying the reciprocal theorem (see §3.3), we arrive at

$$
\int_{S_{p}} \theta \boldsymbol{n} \cdot \boldsymbol{\nabla} \theta^{(0)} \mathrm{d} S=\int_{S_{p}} \theta^{(0)} \boldsymbol{n} \cdot \boldsymbol{\nabla} \theta \mathrm{d} S+\mathrm{Pe} \int_{V} \boldsymbol{u} \cdot \frac{\theta^{(0)} \boldsymbol{\nabla} \theta}{\sqrt{1+2 \beta \theta}} \mathrm{d} V
$$

where, the contributions from surfaces at infinity (denoted by $S_{\infty}$ ) vanish because the integrands decay faster than $\mathrm{d} S$ grows at large distances from the particle. Equation (4.19) can be simplified to

$$
\mathrm{Nu}_{T}=\mathrm{Nu}_{T}^{(0)}+\frac{\mathrm{Pe}}{2 \pi(1+\beta / 2)} \int_{V} \boldsymbol{u} \cdot \frac{\theta^{(0)} \nabla \theta}{\sqrt{1+2 \beta \theta}} \mathrm{d} V .
$$

by applying the boundary conditions on $S_{p}$.

Inspecting Eqs. (4.20), (4.13), (4.11), and (4.10), we determine that

$$
\begin{aligned}
\mathrm{Nu}_{T}^{(1)} & =\frac{1}{2 \pi(1+\beta / 2)} \\
& \times\left[\int_{V} \boldsymbol{u} \cdot \frac{\theta^{(0)} \boldsymbol{\nabla} \theta^{(0)}}{\sqrt{1+2 \beta \theta^{(0)}}} \mathrm{d} V+\boldsymbol{e} \cdot \int_{\mathbb{R}^{3}} \tilde{\theta}^{(0)} \tilde{\boldsymbol{\nabla}}\left(\tilde{\theta}^{(1)}-\tilde{\theta}^{(0)}\right) \mathrm{d} \tilde{\boldsymbol{r}}\right],
\end{aligned}
$$


where the second integral on the right-hand side is over the entire three-dimensional real space $\mathbb{R}^{3}$ and $\tilde{\theta}^{(0)}=\mathrm{Nu}_{T}^{(0)} / 2 \tilde{r}$. We proceed with evaluating the integral appearing first on the right-hand side of Eq. (4.21). To that effect, we convert this volume integral into a pair of surface integrals as

$$
\begin{aligned}
& \int_{V} \boldsymbol{u} \cdot \frac{\theta^{(0)} \boldsymbol{\nabla} \theta^{(0)}}{\sqrt{1+2 \beta \theta^{(0)}}} \mathrm{d} V \\
& =\frac{1}{3 \beta^{2}} \int_{V} \boldsymbol{\nabla} \cdot\left[\sqrt{1+2 \beta \theta^{(0)}}\left(\beta \theta^{(0)}-1\right) \boldsymbol{u}\right] \mathrm{d} V \\
& =-\frac{1}{3 \beta^{2}}\left[\int_{S_{p}} \sqrt{1+2 \beta \theta^{(0)}}\left(\beta \theta^{(0)}-1\right) \boldsymbol{n} \cdot \boldsymbol{u} \mathrm{d} S\right. \\
& \left.\quad+\int_{S_{\infty}} \sqrt{1+2 \beta \theta^{(0)}}\left(\beta \theta^{(0)}-1\right) \boldsymbol{n} \cdot \boldsymbol{u} \mathrm{d} S\right] .
\end{aligned}
$$

Due to no penetration condition, $\boldsymbol{n} \cdot \boldsymbol{u}=0$ on $S_{p}$. Also,

$$
\int_{S_{\infty}} \boldsymbol{n} \cdot \boldsymbol{u} \mathrm{d} S=0
$$

because the flow is incompressible (i.e, $\boldsymbol{\nabla} \cdot \boldsymbol{u}=0$ ). Therefore, the values of both surface integrals in Eq. (4.22) amount to zero, which means that the volume integral is zero, too. Additionally,

$$
\begin{aligned}
& \boldsymbol{e} \cdot \int_{\mathbb{R}^{3}} \tilde{\theta}^{(0)} \tilde{\nabla} \tilde{\theta}^{(0)} \mathrm{d} \tilde{\boldsymbol{r}} \\
& =-\frac{\left(\mathrm{Nu}_{T}^{(0)}\right)^{2}}{4} \int_{0}^{\infty} \frac{1}{\tilde{r}} \int_{0}^{\pi} \sin ^{2} \theta \int_{0}^{2 \pi} \cos \varphi \mathrm{d} \varphi \mathrm{d} \theta \mathrm{d} \tilde{r}=0 .
\end{aligned}
$$

Incorporating these results, we find (after algebraic manipulations) that 


$$
\begin{aligned}
\mathrm{Nu}_{T}^{(1)} & =\frac{\left(\mathrm{Nu}_{T}^{(0)}\right)^{2}}{8 \pi(1+\beta / 2)} \int_{\mathbb{R}^{3}} \frac{\boldsymbol{e} \cdot \tilde{\boldsymbol{r}}}{\tilde{r}^{4}} \exp \left[-\frac{1}{2}(\tilde{r}-\boldsymbol{e} \cdot \tilde{\boldsymbol{r}})\right] \mathrm{d} \tilde{\boldsymbol{r}} \\
& =\frac{\left(\mathrm{Nu}_{T}^{(0)}\right)^{2}}{4(1+\beta / 2)}
\end{aligned}
$$

The evaluation of the volume integral over $\mathbb{R}^{3}$ was detailed in $§ 3.3$.

Replacing for $\mathrm{Nu}_{T}^{(1)}$ in Eq. (4.11), we have

$$
\begin{aligned}
\mathrm{Nu}_{T} & =(1+\beta / 2)\left[\frac{\mathrm{Nu}_{T}^{(0)}}{1+\beta / 2}+\frac{\mathrm{Pe}}{4}\left(\frac{\mathrm{Nu}_{T}^{(0)}}{1+\beta / 2}\right)^{2}+a(\mathrm{Pe})\right] \\
& =(1+\beta / 2)\left[\mathrm{Nu}_{T, 0}^{(0)}+\mathrm{Pe} \frac{\left(\mathrm{Nu}_{T, 0}^{(0)}\right)^{2}}{4}+a(\mathrm{Pe})\right] \\
& =(1+\beta / 2) \mathrm{Nu}_{T, 0}+a(\mathrm{Pe}),
\end{aligned}
$$

where $\mathrm{Nu}_{T, 0}$ and $\mathrm{Nu}_{T, 0}^{(0)}$ are the Nusselt numbers corresponding, respectively, to $\beta=$ 0 and to $\beta=0$ and $\mathrm{Pe}=0$. In other words, the former is the constant conductivity Nusselt number and the latter is the constant conductivity pure conduction Nusselt number. Remember that Eq. (4.12) describes a linear boundary value problem and, therefore, changing its boundary condition on $S_{p}$ from $\theta=1+\beta / 2$ to $\theta=1$ alters the respective Nusselt number by a factor of $(1+\beta / 2)^{-1}$.

Equation (4.26) indicates that, to the leading order in $\mathrm{Pe}, \mathrm{Nu}_{T}$ is the product of a $\beta$-dependent term and the constant-conductivity Nusselt number, which depends on Pe and the geometry of the particle. It is worth emphasizing that this relation was derived with no restriction on the flow Reynolds number, defined as $\operatorname{Re}=\rho U_{\infty} \ell / \mu$. 
Even no-slip condition was not necessary. The only conditions enforced were the flow incompressibility and no flow penetration into the particle. Lastly, we note that Eq. (4.26) recovers the classical result of Brenner [4] for $\beta=0$ and generalizes the work of Polyanin [21] for the special case of Stokes flow (i.e., Re $=0$ ).

\subsubsection{Limit of advection-dominated heat transport}

Suppose the transport of heat is dominated by advection, i.e., Pe $\gg 1$. In this limit, the temperature outside the particle approaches $T_{\infty}$ at short distances from $S_{p}$. In other words, the temperature variations are confined to a narrow layer next to the boundary of the particle. Here, similar to §4.3.1, we aim for developing an asymptotic approximation for the Nusselt number.

Assuming that $S_{p}$ is smooth, we follow Polyanin [49] and adopt a generalized boundary layer coordinate system $(\mathrm{x}, \mathrm{y}, \mathrm{z})$, with the corresponding unit vectors $\boldsymbol{e}_{\mathrm{x}}$, $\boldsymbol{e}_{\mathrm{y}}$, and $\boldsymbol{e}_{\mathrm{z}}$, and associated metric coefficients $h_{\mathrm{x}}, h_{\mathrm{y}}$, and $h_{\mathrm{z}}$. Remember that all lengths are non-dimensionalized by $\ell$. The y component in this system measures the distance from the surface of the particle and, thus, $\boldsymbol{e}_{\mathrm{y}}=\boldsymbol{n}$ at $\mathrm{y}=0$ (i.e., at $S_{p}$ ). The direction of the $\mathrm{x}$ coordinate is chosen to be the same as the component of the fluid velocity vector projected onto the plane normal to $\boldsymbol{e}_{\mathrm{y}}$. The direction of the third coordinate is then determined by $\boldsymbol{e}_{\mathrm{z}}=\boldsymbol{e}_{\mathrm{x}} \times \boldsymbol{e}_{\mathrm{y}}$.

Equation (4.1), written in terms of the $(\mathrm{x}, \mathrm{y}, \mathrm{z})$ coordinates takes the form of

$$
\operatorname{Pe}\left(\frac{u_{\mathrm{x}}}{h_{\mathrm{x}}} \frac{\partial T}{\partial \mathrm{x}}+\frac{u_{\mathrm{y}}}{h_{\mathrm{y}}} \frac{\partial T}{\partial \mathrm{y}}\right)=\frac{1}{h_{\mathrm{x}} h_{\mathrm{y}} h_{\mathrm{z}}}\left\{\frac{\partial}{\partial \mathrm{x}}\left[\frac{h_{\mathrm{y}} h_{\mathrm{z}}}{h_{\mathrm{x}}} \frac{\partial}{\partial \mathrm{x}}\left(T+\frac{\beta}{2} T^{2}\right)\right]\right.
$$




$$
\begin{aligned}
& \left.+\frac{\partial}{\partial \mathrm{y}}\left[\frac{h_{\mathrm{x}} h_{\mathrm{z}}}{h_{\mathrm{y}}} \frac{\partial}{\partial \mathrm{y}}\left(T+\frac{\beta}{2} T^{2}\right)\right]+\frac{\partial}{\partial \mathrm{z}}\left[\frac{h_{\mathrm{x}} h_{\mathrm{y}}}{h_{\mathrm{z}}} \frac{\partial}{\partial \mathrm{z}}\left(T+\frac{\beta}{2} T^{2}\right)\right]\right\} \\
& \text { with } \quad T=1 \quad \text { at } \mathrm{y}=0 \text { and } \quad \lim _{\mathrm{y} \rightarrow \infty} T=0 .
\end{aligned}
$$

In the above equation,

$$
u_{\mathrm{x}}=\frac{1}{h_{\mathrm{y}} h_{\mathrm{z}}} \frac{\partial \Psi}{\partial \mathrm{y}} \quad \text { and } \quad u_{\mathrm{y}}=-\frac{1}{h_{\mathrm{x}} h_{\mathrm{z}}} \frac{\partial \Psi}{\partial \mathrm{x}}
$$

are the velocity components in the $\mathrm{x}$ and $\mathrm{y}$ directions, respectively, where $\Psi$ is a pseudo stream function. Note that $u_{z}$ is zero per the definition of the generalized boundary layer coordinate system. Given that the distribution of temperature is restricted to a thin region close to $S_{p}$, it is convenient to expand the metric coefficients and $\Psi$ about $\mathrm{y}=0$ as

$$
\begin{aligned}
& h_{\mathrm{x}}=h_{\mathrm{x}, 0}(\mathrm{x}, \mathrm{z})+h_{\mathrm{x}, 1}(\mathrm{x}, \mathrm{z}) \mathrm{y}+\cdots, \\
& h_{\mathrm{y}}=1, \\
& h_{\mathrm{z}}=h_{\mathrm{z}, 0}(\mathrm{x}, \mathrm{z})+h_{\mathrm{z}, 1}(\mathrm{x}, \mathrm{z}) \mathrm{y}+\cdots, \\
& \Psi=\frac{1}{2} \Psi_{2}(\mathrm{x}, \mathrm{z}) \mathrm{y}^{2}+\cdots,
\end{aligned}
$$

where

$$
\Psi_{2}=\left(h_{\mathrm{z}} \frac{\partial u_{\mathrm{x}}}{\partial \mathrm{y}}\right)_{\mathrm{y}=0}=h_{\mathrm{z}, 0} \tau_{0}
$$

with $\tau_{0}$ being the dimensionless shear stress at the surface of the particle. To ensure the validity of Eq. (4.32), the Prandtl number $\operatorname{Pr}=c_{p} \mu / k_{0}$ is assumed to be large. 
Needless to say, both $\Psi$ and $\partial \Psi / \partial \mathrm{y}$ are zero at $\mathrm{y}=0$, due to the no-slip condition.

The thickness of the temperature boundary layer is known to scale with $\mathrm{Pe}^{-1 / 3}$, which results from equating the order of magnitude of the advective and conductive terms within the boundary layer (see, e.g., $[6,34]$ ). Considering this scaling and the structure of Eq. (4.27), we proceed with expanding $T$ and $\mathrm{Nu}_{T}$ as

$$
\begin{aligned}
T & =T^{(0)}(\mathrm{x}, \tilde{\mathrm{y}}, \mathbf{z})+\mathcal{O}\left(\mathrm{Pe}^{-1 / 3}\right), \\
\mathrm{Nu}_{T} & =\mathrm{Pe}^{1 / 3} \mathrm{Nu}_{T}^{(0)}+\mathcal{O}(1)
\end{aligned}
$$

and with making the following change of variables:

$$
\begin{aligned}
& \mathrm{Y}=\mathrm{Pe}^{1 / 3} \mathrm{y}, \\
& \mathrm{X}=\int_{\mathrm{x}_{0}}^{\mathrm{x}} h_{\mathrm{x}, 0} h_{\mathrm{z}, 0} \mathrm{~d} \hat{\mathrm{x}} .
\end{aligned}
$$

Next, we rewrite Eq. (4.27) to the leading order in Pe as

$$
\begin{aligned}
& \mathrm{Y} \Psi_{2} \frac{\partial T^{(0)}}{\partial \mathrm{X}}-\frac{\mathrm{Y}^{2}}{2} \frac{\partial \Psi_{2}}{\partial \mathrm{X}} \frac{\partial T^{(0)}}{\partial \mathrm{Y}}=\frac{\partial^{2}}{\partial \mathrm{Y}^{2}}\left[T^{(0)}+\frac{\beta}{2}\left(T^{(0)}\right)^{2}\right] \\
& \text { with } T^{(0)}=1 \quad \text { at } \mathrm{Y}=0 \text { and } \lim _{\mathrm{Y} \rightarrow \infty} T^{(0)}=0 .
\end{aligned}
$$

Equation (4.38) can be further simplified to its self-similar form

$$
\begin{aligned}
& \frac{d^{2}}{d \eta^{2}}\left[T^{(0)}+\frac{\beta}{2}\left(T^{(0)}\right)^{2}\right]+3 \eta^{2} \frac{d T^{(0)}}{d \eta}=0 \quad \text { with } \\
& T^{(0)}=1 \quad \text { at } \quad \eta=0 \text { and } \quad \lim _{\eta \rightarrow \infty} T^{(0)}=0,
\end{aligned}
$$


where the new independent variable $\eta$ is defined, according to the von Mises transformation, via

$$
\eta=\sqrt{\Psi_{2}}\left(9 \int_{\mathrm{x}_{0}}^{\mathrm{X}} \sqrt{\Psi_{2}} d \hat{\mathrm{X}}\right)^{-1 / 3} \mathrm{Y} .
$$

Taking everything into account, the leading-order term in the Nusselt number expansion can be expressed as

$$
\begin{aligned}
\mathrm{Nu}_{T}^{(0)} & =-\left.\left(\frac{1+\beta}{2 \pi}\right) \int_{S_{p}} \frac{\partial T^{(0)}}{\partial \tilde{\mathrm{y}}}\right|_{\tilde{\mathbf{y}}=0} \mathrm{~d} S \\
& =-\left.\left(\frac{1+\beta}{4 \pi}\right) \frac{d T^{(0)}}{d \eta}\right|_{\eta=0} \int_{\mathbf{z}_{\min }}^{\mathbf{z}_{\max }}\left(\int_{\mathbf{x}_{\text {min }}}^{\mathbf{x}_{\text {max }}} \sqrt{3 \Psi_{2}} \mathrm{dX}\right)^{2 / 3} \mathrm{~d} \mathbf{z} \\
& =\left.(1+\beta) \frac{d T^{(0)}}{d \eta}\right|_{\eta=0}\left(\left.\frac{d T^{(0)}}{d \eta}\right|_{\eta=0, \beta=0}\right)^{-1} \mathrm{Nu}_{T, 0}^{(0)} \\
& =-\left.\Gamma\left(\frac{4}{3}\right)(1+\beta) \frac{d T^{(0)}}{d \eta}\right|_{\eta=0} \mathrm{Nu}_{T, 0}^{(0)},
\end{aligned}
$$

where $\mathrm{Nu}_{T, 0}^{(0)}$ represents the value of $\mathrm{Nu}_{T}^{(0)}$ corresponding to $\beta=0$. Of course, Eq. (4.41) reproduces the classical results of Lighthill [1] and Acrivos [40].

Below we calculate the prefactor $c$ :

$$
c=-\left.\Gamma\left(\frac{4}{3}\right)(1+\beta) \frac{d T^{(0)}}{d \eta}\right|_{\eta=0},
$$

where $T^{(0)}$ satisfies Eq. (4.39). We first consider the limit of $\beta \ll 1$ and proceed with a regular perturbation expansion of $T^{(0)}$ as

$$
T^{(0)}=T_{0}^{(0)}+\beta T_{1}^{(0)}+\mathcal{O}\left(\beta^{2}\right) .
$$


Substituting Eq. (4.43) in Eq. (4.39), we find that

$$
\begin{aligned}
& \frac{d^{2} T_{0}^{(0)}}{d \eta^{2}}+3 \eta^{2} \frac{d T_{0}^{(0)}}{d \eta}=0 \text { with } \\
& T_{0}^{(0)}=1 \quad \text { at } \quad \eta=0 \text { and } \lim _{\eta \rightarrow \infty} T_{0}^{(0)}=0
\end{aligned}
$$

and

$$
\begin{aligned}
& \frac{d^{2} T_{1}^{(0)}}{d \eta^{2}}+3 \eta^{2} \frac{d T_{1}^{(0)}}{d \eta}+\frac{1}{2} \frac{d^{2}}{d \eta^{2}}\left(T_{0}^{(0)}\right)^{2}=0 \text { with } \\
& T_{1}^{(0)}=0 \quad \text { at } \eta=0 \text { and } \lim _{\eta \rightarrow \infty} T_{1}^{(0)}=0 .
\end{aligned}
$$

The solution of Eq. (4.44) is

$$
T_{0}^{(0)}=1-\frac{1}{\Gamma(4 / 3)} \int_{0}^{\eta} \exp \left(-\hat{\eta}^{3}\right) \mathrm{d} \hat{\eta}
$$

which is derived using the integrating factor method. Applying the same approach to Eq. (4.45), we obtain (after some mathematical manipulations)

$$
\begin{aligned}
T_{1}^{(0)}= & \left.\frac{d T_{1}^{(0)}}{d \eta}\right|_{\eta=0} \Gamma(4 / 3)\left(1-T_{0}^{(0)}\right)-\frac{\left(1-T_{0}^{(0)}\right)^{2}}{2} \\
- & \frac{1}{[\Gamma(4 / 3)]^{2}} \int_{0}^{\eta} \exp \left(-\hat{\eta}^{3}\right) \\
& \times\left[\Gamma(4 / 3) \hat{\eta}^{3} T_{0}^{(0)}+\int_{0}^{\hat{\eta}} \hat{\hat{\eta}}^{3} \exp \left(-\hat{\hat{\eta}}^{3}\right) \mathrm{d} \hat{\hat{\eta}}\right] \mathrm{d} \hat{\eta},
\end{aligned}
$$

where the first term on the right-hand side is numerically calculated (by enforcing the boundary condition at infinity) to be 


$$
\left.\frac{d T_{1}^{(0)}}{d \eta}\right|_{\eta=0}=0.667
$$

Given Eqs. (4.43), (4.46), and (4.48), for small $\beta$, we can write

$$
\begin{aligned}
c & =1+\left[1-\left.\Gamma\left(\frac{4}{3}\right) \frac{d T_{1}^{(0)}}{d \eta}\right|_{\eta=0}\right] \beta+\mathcal{O}\left(\beta^{2}\right) \\
& =1+0.404 \beta+\mathcal{O}\left(\beta^{2}\right) .
\end{aligned}
$$

Now, suppose $\beta \gg 1$, while $\beta \ll \operatorname{Pe}$ and $\beta \ll \operatorname{Pr}$. We realize, through inspecting Eq. (4.39), that $d T^{(0)} / d \eta$ scales with $\beta^{-1 / 3}$ in this limit. Informed by this scaling, we expand $T^{(0)}$ as

$$
T^{(0)}=T_{0}^{(0)}+\mathcal{O}\left(\beta^{-1 / 3}\right)
$$

and introduce

$$
\tilde{\eta}=\beta^{-1 / 3} \eta
$$

Replacing for $T^{(0)}$ and $\eta$ in Eq. (4.39), we then arrive at

$$
\begin{aligned}
& \frac{d^{2}}{d \tilde{\eta}^{2}}\left[\left(T_{0}^{(0)}\right)^{2}\right]+6 \tilde{\eta}^{2} \frac{d T_{0}^{(0)}}{d \tilde{\eta}}=0 \text { with } \\
& T_{0}^{(0)}=1 \quad \text { at } \tilde{\eta}=0 \text { and } \lim _{\tilde{\eta} \rightarrow \infty} T_{1}^{(0)}=0 .
\end{aligned}
$$

We solve the above nonlinear ordinary differential equation numerically and determine that

$$
c=-\left.\Gamma\left(\frac{4}{3}\right) \frac{d T_{0}^{(0)}}{d \tilde{\eta}}\right|_{\tilde{\eta}=0} \beta^{2 / 3}+\mathcal{O}\left(\beta^{1 / 3}\right)
$$




$$
=0.710 \beta^{2 / 3}+\mathcal{O}\left(\beta^{1 / 3}\right) .
$$

What is truly surprising, based on the results of Eqs. (4.49) and (4.53), is that the prefactor $c$ is very well approximated by a single formula (for the entire range of $\beta$ )

as

$$
c \approx\left(1+\frac{3 \beta}{5}\right)^{2 / 3}
$$

Note that the above expression captures both low- and high- $\beta$ asymptotes remarkably well. Hence, we obtain

$$
\mathrm{Nu}_{T} \approx\left(1+\frac{3 \beta}{5}\right)^{2 / 3} \mathrm{Nu}_{T, 0}+\mathcal{O}(1)
$$

with $\mathrm{Nu}_{T, 0}$ denoting the Nusselt number for the case of $\beta=0$.

\subsubsection{Bridging results for limits of low and high Péclet numbers}

The results of the previous two subsections can be described succinctly as

$$
\left.\begin{array}{lll}
\frac{\mathrm{Nu}_{T}}{\mathrm{Nu}_{T, 0}} \approx\left(1+\frac{\beta}{2}\right) & \text { for } & \mathrm{Pe} \ll 1 \\
\frac{\mathrm{Nu}_{T}}{\mathrm{Nu}_{T, 0}} \approx\left(1+\frac{3 \beta}{5}\right)^{2 / 3} & \text { for } & \mathrm{Pe} \gg 1
\end{array}\right\} \Rightarrow \frac{\mathrm{Nu}_{T}}{\mathrm{Nu}_{T, 0}} \approx(1+a \beta)^{b},
$$

where $1 / 2 \lesssim a \lesssim 3 / 5$ and $2 / 3 \lesssim b \lesssim 1$. The functional dependency of these two parameters on the Péclet number may be formulated as

$$
a \approx \frac{(3 / 5) \sqrt{\mathrm{Pe}}+a_{0}}{\sqrt{\mathrm{Pe}}+2 a_{0}},
$$




$$
b \approx \frac{(2 / 3) \sqrt{\mathrm{Pe}}+b_{0}}{\sqrt{\mathrm{Pe}}+b_{0}}
$$

where

$$
a_{0}=5.78 \text { and } b_{0}=5.90
$$

The above relations are motivated by the full numerical solution of Eq. (4.1), subject to the boundary conditions in Eq. (4.2), for several basic particle shapes. The accuracy of the predictions made by Eqs. (4.56)-(4.59) and the details of the numerical solutions will be discussed in $\S 4.5$. Note that, for some simple geometries, approximate formulas are available for $\mathrm{Nu}_{T, 0}$ (see, e.g., §2).

Overall, our asymptotic analyses in this section (summarized by Eq. (4.56)) suggest that the ratio between the Nusselt number and its corresponding value for the case of constant conductivity (i.e., $\beta=0$ ) is approximately equal to a term that is mainly a function of $\beta$, while being weakly dependent on Pe. It is important to emphasize that this result was derived for particles of arbitrary shape under a fairly general flow condition, namely a laminar, steady-state, incompressible flow. We have only demanded the Prandtl number to be large when the Péclet number is high.

\subsection{Variation of Nusselt number for uniform heat flux boundary condition}

In $\S 4.3$, we considered Eq. (4.1) assuming that the surface of the particle is held at a constant temperature (see Eq. (4.2)). There, the assumption of a Dirichlet boundary 
condition on $S_{p}$ allowed us to effectively employ the Kirchhoff and von Mises transformations (for, respectively, $\mathrm{Pe} \ll 1$ and $\mathrm{Pe} \gg 1$ ) to develop a nearly analytical formula for $\mathrm{Nu}_{T}$. Unfortunately, neither techniques can be directly applied to find the general form of the Nusselt number when a uniform heat flux is imposed on $S_{p}$. That a Neumann boundary condition is more challenging to deal with analytically than its Dirichlet counterpart is a known matter (see, e.g., §2 and §3). Despite this difficulty, we derive an estimate for $\mathrm{Nu}_{Q}$ with the aid of the following scaling arguments.

\subsubsection{Limit of conduction-dominated heat transport}

Consider the limit of $\mathrm{Pe} \ll 1$, and, accordingly, an inner expansion of the form

$$
T=T^{(0)}+\operatorname{Pe} T^{(1)}+\cdots
$$

for the temperature, where

$$
\begin{aligned}
& \boldsymbol{\nabla} \cdot\left[\left(1+\beta T^{(0)}\right) \nabla T^{(0)}\right]=0 \quad \text { with } \\
& \left(1+\beta T^{(0)}\right)\left(\boldsymbol{n} \cdot \nabla T^{(0)}\right)=-1 \quad \text { for } \quad \boldsymbol{r} \in S_{p} \quad \text { and } \quad \lim _{r \rightarrow \infty} T^{(0)}=0
\end{aligned}
$$

and

$$
\begin{aligned}
\boldsymbol{u} \cdot \boldsymbol{\nabla} T^{(0)} & =\nabla^{2}\left(T^{(1)}+\beta T^{(0)} T^{(1)}\right) \quad \text { with } \\
\boldsymbol{n} \cdot \boldsymbol{\nabla}\left(T^{(1)}+\beta T^{(0)} T^{(1)}\right) & =0 \quad \text { for } \quad \boldsymbol{r} \in S_{p} .
\end{aligned}
$$

Applying the Kirchhoff transformation (see Eq. (4.7)), it can be shown that 


$$
T^{(0)}=\frac{\sqrt{1+2 \beta \theta^{(0)}}-1}{\beta},
$$

where

$$
\begin{aligned}
\nabla^{2} \theta^{(0)} & =0 \quad \text { with } \\
\boldsymbol{n} \cdot \boldsymbol{\nabla} \theta^{(0)} & =-1 \quad \text { for } \quad \boldsymbol{r} \in S_{p} \quad \text { and } \quad \lim _{r \rightarrow \infty} \theta^{(0)}=0 .
\end{aligned}
$$

Additionally, consistent with Eq. (4.60), the Nusselt number can be expressed as

$$
\begin{aligned}
\mathrm{Nu}_{Q} & =\frac{\mathbb{S}_{p}}{2 \pi \bar{T}^{(0)}}\left(1-\mathrm{Pe} \frac{\bar{T}^{(1)}}{\bar{T}^{(0)}}\right)+\cdots \\
& =\mathrm{Nu}_{Q}^{(0)}+\mathrm{PeNu}_{Q}^{(1)}+\cdots
\end{aligned}
$$

where the overbar indicates average over $S_{p}$ (see also Eq. (4.71)). The zeroth-order Nusselt number is determined by the solution of Eq. (4.64), with its dependency on $\beta$ obeying Eq. (4.63). How about the dependency of $\mathrm{Nu}_{Q}^{(1)}$ on $\beta$ ? To find this out, we examine the limits of small and large $\beta$.

Suppose $\beta \ll 1$, in which case we can write

$$
\mathrm{Nu}_{Q}^{(1)}=\mathrm{Nu}_{Q, 0}^{(1)}+\beta \mathrm{Nu}_{Q, 1}^{(1)}+\cdots
$$

Since both $\mathrm{Pe}$ and $\beta$ are small, then, we need to only retain $\mathrm{Nu}_{Q, 0}^{(1)}$ in the above expansion. This means that, to the leading order, $\mathrm{Nu}_{Q}^{(1)}$ is independent of $\beta$. Now, assume $\beta \gg 1$. Equations (4.63) and (4.62) suggest that, in this limit,

$$
T^{(0)} \sim \frac{1}{\sqrt{\beta}} \quad \text { and } \quad T^{(1)} \sim \frac{1}{\beta}
$$


Hence, we conclude that, again, to the leading order, $\mathrm{Nu}_{Q}^{(1)}$ is independent of $\beta$. Given the behavior of $\mathrm{Nu}_{Q}^{(1)}$ in its asymptotic limits, it is reasonable to approximate $\mathrm{Nu}_{Q}$ for small Péclet numbers as a summation of two terms: one that only depends on $\beta$ and another one that solely changes with Pe.

\subsubsection{Limit of advection-dominated heat transport}

Consider the limit of $\mathrm{Pe} \gg 1$, where the thermal boundary layer shrinks at a rate proportional to $\mathrm{Pe}^{-1 / 3}$. In this case, the temperature and Nusselt number can be described as (see, e.g., [59])

$$
\begin{aligned}
T & =\mathrm{Pe}^{-1 / 3} T^{(0)}+\mathrm{Pe}^{-2 / 3} T^{(1)}+\cdots, \\
\mathrm{Nu}_{Q} & =\frac{\mathbb{S}_{p}}{2 \pi \bar{T}^{(0)}}\left(\mathrm{Pe}^{1 / 3}-\frac{\bar{T}^{(1)}}{\bar{T}^{(0)}}\right)+\cdots .
\end{aligned}
$$

where, upon substituting for $T$ in Eqs. (4.1) and (4.3), we have (in generalized boundary layer coordinates)

$$
\begin{aligned}
& \mathrm{Y} \Psi_{2} \frac{\partial T^{(0)}}{\partial \mathrm{X}}-\frac{\mathrm{Y}^{2}}{2} \frac{\partial \Psi_{2}}{\partial \mathrm{X}} \frac{\partial T^{(0)}}{\partial \mathrm{Y}}=\frac{\partial^{2} T^{(0)}}{\partial \mathrm{Y}^{2}} \\
& \text { with } \frac{\partial T^{(0)}}{\partial \mathrm{Y}}=-1 \quad \text { at } \quad \mathrm{Y}=0 \quad \text { and } \quad \lim _{\mathrm{Y} \rightarrow \infty} T^{(0)}=0
\end{aligned}
$$

Equation (4.70) does not depend on $\beta$ and, in fact, is identical to the one for $\beta=0$.

Recognizing that $\bar{T}^{(0)}$ is not a function of $\beta$, we deduce from Eq. (4.69) that $\mathrm{Nu}_{Q}$ for large Péclet numbers is approximately equal to the sum of a Pe-dependent term and one that only varies with $\beta$. 


\subsubsection{Bridging results for limits of low and high Péclet numbers}

The main takeaway point of our scaling analyses in $\S 4.4 .1$ and $\S 4.4 .2$ is that $\mathrm{Nu}_{Q}$ may be estimated as the summation of two terms, one of which is a function of Pe and the other one is a function of $\beta$. As a unified formula, we, therefore, propose

$$
\mathrm{Nu}_{Q} \approx \mathrm{Nu}_{Q, 0}+\left(\mathrm{Nu}_{Q}^{c}-\mathrm{Nu}_{Q, 0}^{c}\right)
$$

where $\mathrm{Nu}_{Q, 0}$ represents the value of $\mathrm{Nu}_{Q}$ for $\beta=0$ (i.e., for constant conductivity) and the superscript $c$ denotes the Nusselt number corresponding to $\mathrm{Pe}=0$ (i.e, conduction Nusselt number). The above approximation possesses the required form and has zero error for the cases of $\mathrm{Pe}=0$ and $\beta=0$. We will test the validity of Eq. (4.71) in $\S 4.5$.

Before we conclude this section, it is worth emphasizing that, for certain geometries, fairly accurate estimates of $\mathrm{Nu}_{Q, 0}$ exist in the literature (see, e.g., §2). Furthermore, we have discovered empirically that

$$
\begin{aligned}
\mathrm{Nu}_{Q}^{c} & =\frac{\mathbb{S}_{p} \beta}{2 \pi}\left(\sqrt{\sqrt{1+2 \beta \theta^{(0)}}}-1\right)^{-1} \\
& \approx \frac{\mathbb{S}_{p} \beta}{2 \pi}\left(\sqrt{1+2 \beta \overline{\theta^{(0)}}}-1\right)^{-1}=\frac{\mathbb{S}_{p} \beta}{2 \pi}\left(\sqrt{1+\frac{\mathbb{S}_{p} \beta}{\pi \mathrm{Nu}_{Q, 0}^{c}}}-1\right)^{-1} .
\end{aligned}
$$

Equation (4.72) is exact for a spherical particle and found to be unexpectedly precise for other particle shapes such as ellipsoids, cylinders, cones, and cubes. 


$$
\beta=0.1-\beta=1 \quad-\beta=10
$$

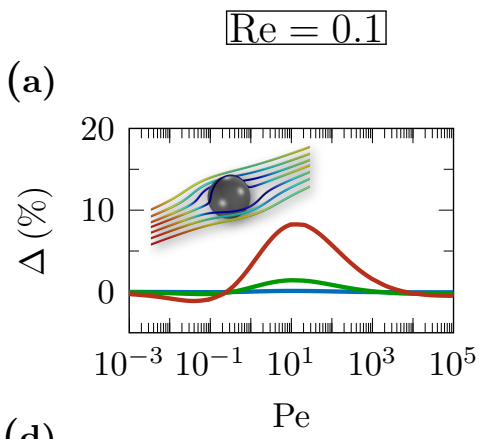

(d)

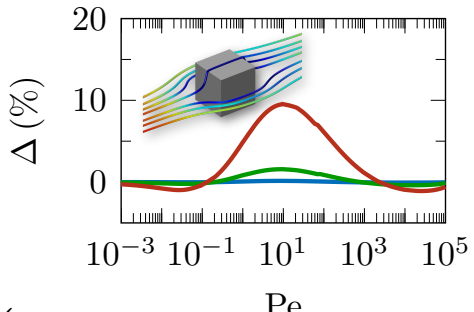

(g)

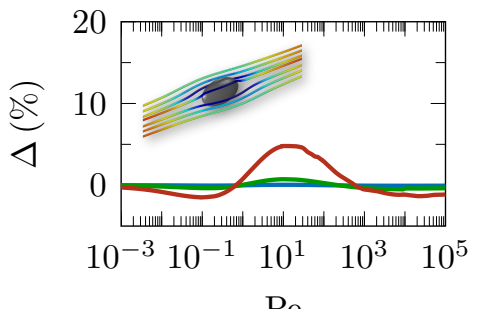

(j)

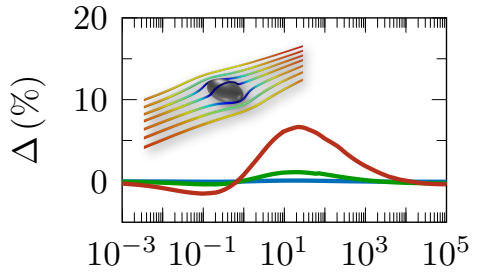

(m)

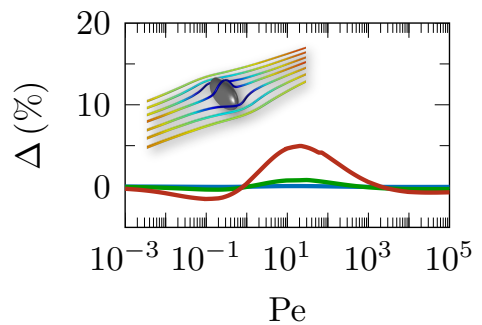

$\mathrm{Re}=1$

(b)

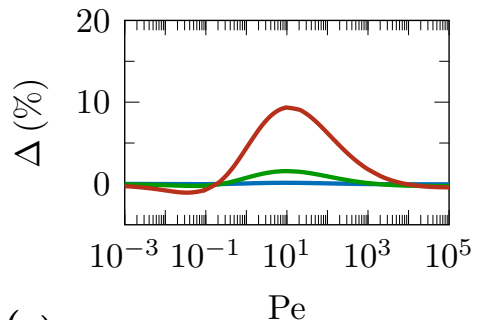

(e)

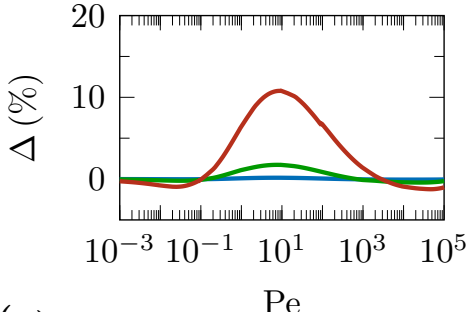

(h)

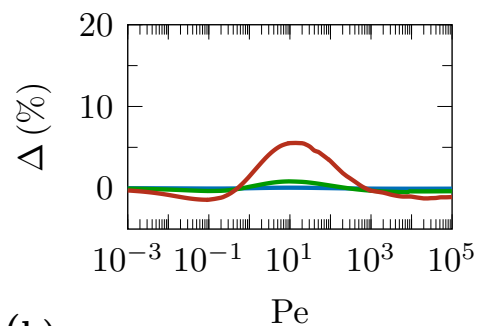

(k)

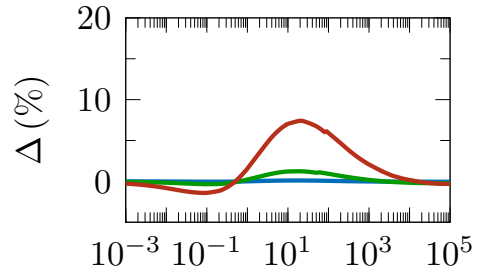

(n)

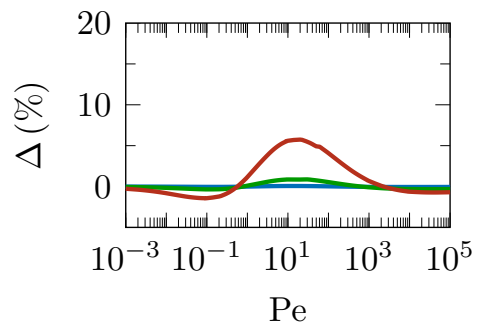

(c)

$\mathrm{Re}=10$

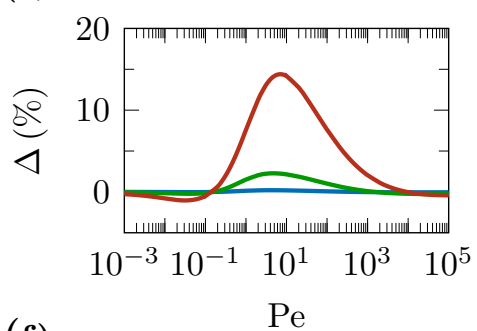

(f)

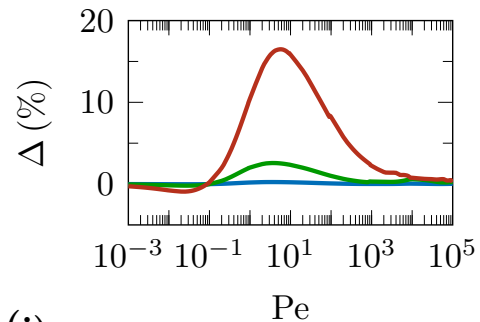

(i)

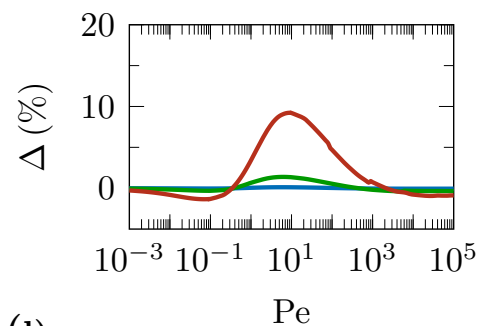

(1)

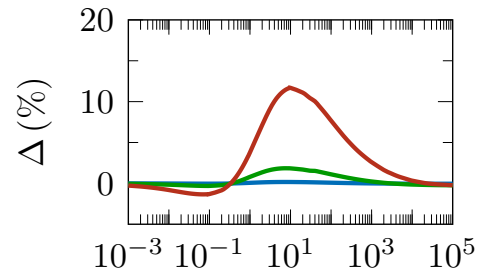

(o)

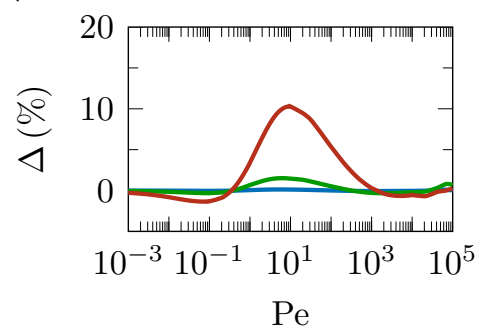

Figure 4.1: Percent difference $\Delta$ between the results of full numerical simulations and the predictions of Eq. (4.56) for $\mathrm{Nu}_{T}$. 


$$
-\beta=0.1-\beta=1 \quad-\beta=10
$$

$$
\mathrm{Re}=0.1
$$

(a)

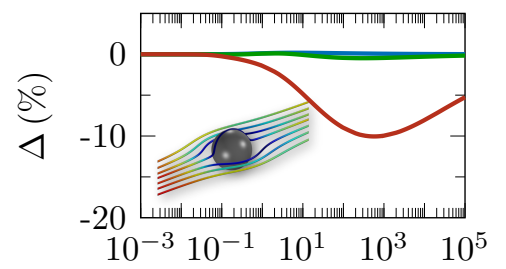

(d)

$\mathrm{Pe}$

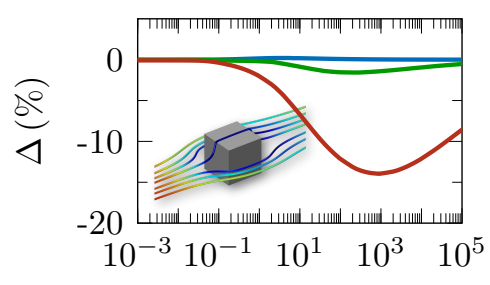

(g)

$\mathrm{Pe}$

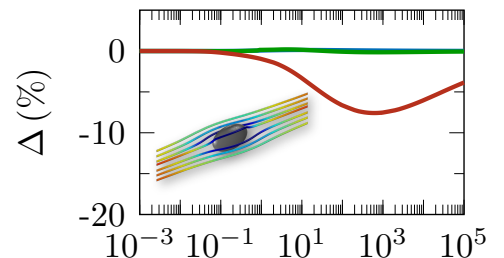

(j)

$\mathrm{Pe}$

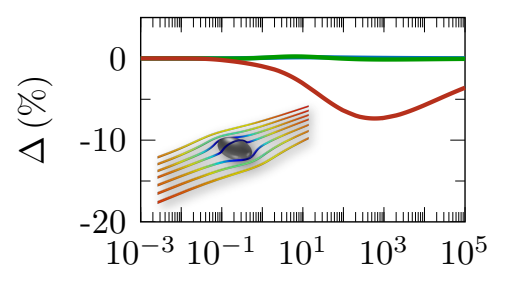

(m)

$\mathrm{Pe}$

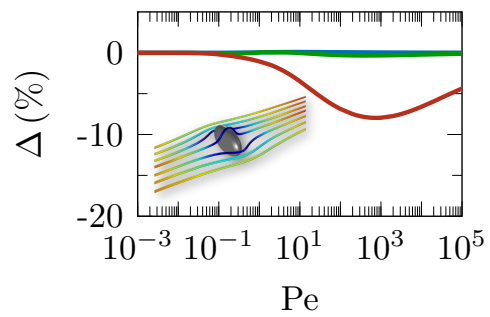

$\mathrm{Re}=1$

(b)

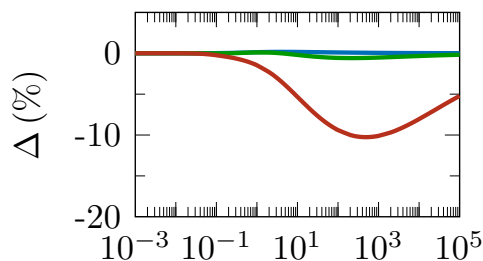

(e)

$\mathrm{Pe}$

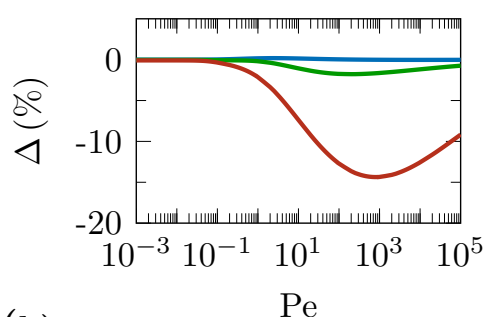

(h)

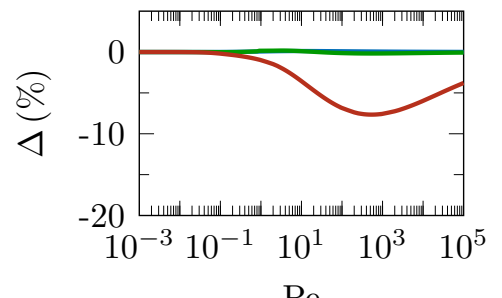

(k)

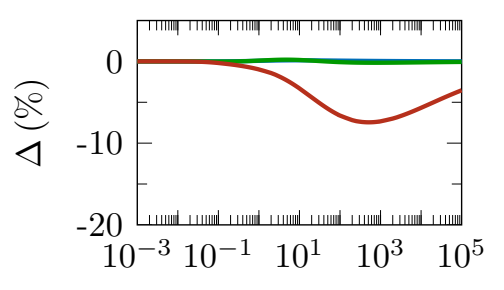

(n)

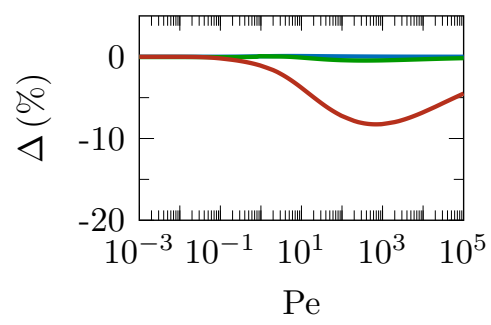

(c)

$\mathrm{Re}=10$

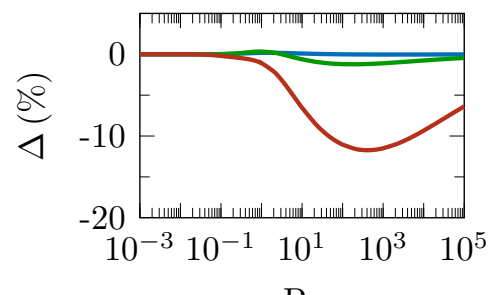

(f)

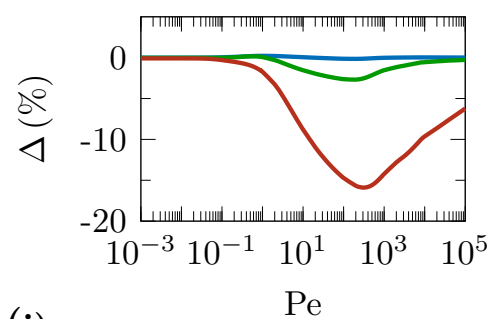

(i)

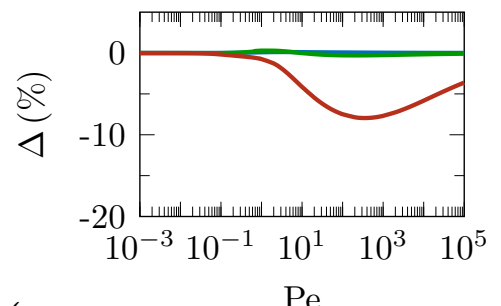

(1)

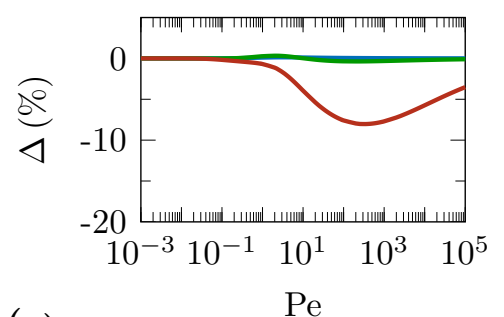

(o)

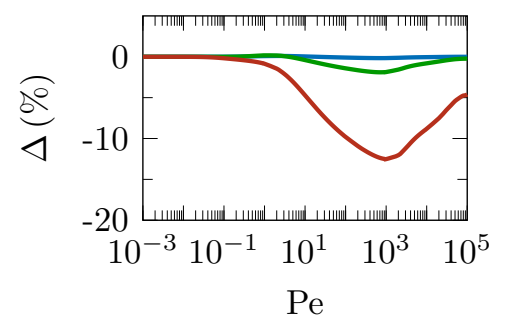

Figure 4.2: Percent difference $\Delta$ between the results of full numerical simulations and the predictions of Eq. (4.71) for $\mathrm{Nu}_{Q}$. 


\subsection{Comparison with direct numerical solutions}

In $\S 4.3$ and $\S 4.4$, we presented approximate formulations for $\mathrm{Nu}_{T}$ and $\mathrm{Nu}_{Q}$ via perturbation analyses in the asymptotic limits of $\mathrm{Pe}$ and $\beta$. To give an idea about the estimation error of the proposed formulas, here, we compare their predictions for spherical, cubic, and ellipsoidal particles against the results obtained from the full numerical solution of the problems described in $\S 4.2$. A finite-element approach, as implemented in COMSOL Multiphysics [60,61], is employed to carry out the computations. We first solve the steady-state incompressible Navier-Stokes equations for the fluid flow, and, then, use the calculated velocity field $\boldsymbol{u}$ to compute the solution of Eq. (4.1) for the temperature distribution $T$. The flow and advection-diffusion equations are solved iteratively, while discretized by $\mathrm{P} 2+\mathrm{P} 1$ and quadratic Lagrange schemes, respectively. The outer boundary at infinity is modeled as a large sphere, whose center coincides with the center of the particle. Specifically, the diameter of the sphere is set to 200 times the characteristic length of the particle. We use tetrahedral elements to mesh the computational domain such that the grid density is the highest near the particle. Grid-independence studies are performed to ensure that the results change only marginally if the mesh is globally refined.

Figures 4.1 and 4.2 show the outcome of our calculations for $\mathrm{Nu}_{T}$ and $\mathrm{Nu}_{Q}$, respectively. The results are presented in the form of $\Delta$ versus Pe plots for $\beta=0.1,1,10$ and $\operatorname{Re}=0.1,1,10$, where the parameter $\Delta$ is defined as the percent difference between the directly computed and predicted values of the Nusselt number. Each figure 
consists of fifteen sub-figures that are organized into five rows and three columns. The sub-figures in the left, middle, and right columns correspond to $\operatorname{Re}=0.1,1$, and 10, respectively. Also, those in the first and second rows belong to spherical and cubic particles, whereas the rest are for an ellipsoidal particle of semi-axis lengths $\mathfrak{a}$, $\mathfrak{b}$, and $\mathfrak{c}$, where $\mathfrak{b} / \mathfrak{a}=2 / 3$ and $\mathfrak{c} / \mathfrak{a}=1 / 3$. Within the bottom three rows, the plots in the first, second, and third rows are for flows along the principal axes of the ellipsoid associated with $\mathfrak{a}, \mathfrak{b}$, and $\mathfrak{c}$, respectively. Note that, in calculating the Nusselt and Péclet numbers the characteristic length $\ell$ is set to the radius for the sphere, to half the side length for the cube, and to the largest semi-axis for the ellipsoid.

Overall, we see that the predictions of Eqs. (4.56) and (4.71) are quite accurate, with the absolute value of $\Delta$ being less than $16.5 \%$ for all the cases considered. The approximations are more precise for particles with more streamlined shapes. Also, as expected, the estimations deviate the most from the numerical results when $\beta$ and Re are large and $\mathrm{Pe}$ is in the intermediate range. Perhaps surprisingly, however, $\Delta$ is very small for $\beta \lesssim \mathcal{O}(1)$, irrespective of its corresponding Reynolds and Péclet numbers. Another observation that can be made is that $\Delta$ is mostly positive in the plots of Fig. 4.1 whereas it is mainly negative in those of Fig. 4.2. Note that $\Delta$ is defined such that it is positive when the predicted Nusselt number overestimates the numerically calculated one. Lastly, for the same shape, Re, and $\beta$, the Péclet number at which the approximation error is maximum is generally higher for $\mathrm{Nu}_{Q}$ than it is for $\mathrm{Nu}_{T}$. 


\subsection{Summary}

We studied the problem of forced convection heat transfer from a particle of arbitrary shape immersed in an unbounded fluid whose thermal conductivity varies linearly with the temperature. Assuming a uniform free-stream flow, we employed asymptotic as well as scaling analyses to develop approximate relations for the variations of the Nusselt number with the Péclet number and the slope of the (normalized) conductivity-temperature curve. We considered both constant temperature and uniform heat flux boundary conditions on the surface of the particle, and discovered that, for the former, $\mathrm{Nu}_{T}$ can be estimated as a product of a Pe-dependent term and one that primarily changes with $\beta$. We also found that, for the latter, $\mathrm{Nu}_{Q}$ may be approximated as a sum of a Pe-dependent piece and a $\beta$-dependent one. In a nutshell, our derivations offer a straightforward way to estimate the Nusselt number for any $\beta$ by just knowing the the Nusselt number corresponding to $\beta=0$, i.e., the constant conductivity Nusselt number.

We evaluated the generality and accuracy of our formulations by comparing their

predictions for $\mathrm{Nu}_{T}$ and $\mathrm{Nu}_{Q}$ with those calculated based on direct numerical solutions of the governing equations. The comparisons confirmed that the proposed approximations are valid over a wide range of parameters. More specifically, they demonstrated that the estimation errors are remarkably low when $\beta \lesssim \mathscr{O}(1)$. 


\section{Chapter 5: Summary and future directions}

We theoretically examined three fundamental problems on the topic of heat transfer from isolated objects, namely (i) conduction heat transfer from oblate spheroids and bispheres, (ii) forced convection heat transfer from an arbitrarily-shaped particle, and (iii) heat transfer from a single object in laminar flows of a variable thermal conductivity fluid. We had noticed that although there have been many studies on mathematical modeling of heat transfer from objects of various shapes (or analogous problems in mass transfer, electrostatics, etc.), a large number of these investigations have focused on the isothermal (Dirichlet) boundary condition and a relatively small number have dealt with the uniform flux (Neumann) boundary condition. In an attempt to partially fill this gap in the literature, in this dissertation, we particularly considered this former condition in our analyses, and demonstrated that mathematical derivation under this choice becomes often more challenging compared to the situation where an isothermal surface condition is considered, which might explain why the Neumann boundary condition has been frequently overlooked.

We employed the tools of applied mathematics to study the above-mentioned problems. Specifically, the methods of separation of variables and eigenfuction expansion were used to solve the Laplace equation in curvilinear coordinate systems for 
the conduction problems. The convection problems, on the other hand, were treated via a combination of the perturbation and boundary layer theories, reciprocal theorem, and scaling arguments. In all cases, we developed precise, but straightforward, approximations for the Nusselt number that can be readily used by engineers to design new thermal systems or better understand the function of the existing ones. The accuracy of the calculated estimations were confirmed through comparison with full analytical or numerical solution of the corresponding transport equations. It is worth emphasizing that the formulas derived for the Nusselt number in this work are all equally applicable for approximating the Sherwood number in equivalent mass transfer problems.

Finally, we note that the mathematical techniques presented in $\S 3$ and $\S 4$ furnish a sound basis for future theoretical investigations on heat transfer from multiple particles, which can be considered as a natural extension of our study. We can envision using the method of reflections in addition to the approaches discussed here to construct asymptotic expressions for the Nusselt number of multi-particle systems. Furthermore, our work in $\S 4$ can be extended to cases where the thermal conductivity is a non-linear function of the temperature and where other fluid properties such as viscosity also vary with the temperature. 


\section{Bibliography}

[1] M. J. Lighthill, "Contributions to the theory of heat transfer through a laminar boundary layer," Proc. Roy. Soc. (London), vol. 202, no. 1070, pp. 359-377, 1950.

[2] A. Acrivos, "Solution of the laminar boundary layer energy equation at high prandtl numbers," Phys. Fluids, vol. 3, no. 4, pp. 657-658, 1960.

[3] A. Acrivos and T. D. Taylor, "Heat and mass transfer from single spheres in stokes flow," Phys. Fluids, vol. 5, no. 4, pp. 387-394, 1962.

[4] H. Brenner, "Forced convection heat and mass transfer at small Peclet numbers from a particle of arbitrary shape," Chem. Eng. Sci., vol. 18, no. 2, pp. 109-122, 1963.

[5] T. D. Taylor, "Heat transfer from single spheres in a low reynolds number slip flow," Phys. Fluids, vol. 6, no. 7, pp. 987-992, 1963.

[6] A. Acrivos and J. D. Goddard, "Asymptotic expansions for laminar forcedconvection heat and mass transfer," J. Fluid Mech., vol. 23, no. 2, pp. 273-291, 1965. 
[7] J. D. Goddard and A. Acrivos, "Asymptotic expansions for laminar forcedconvection heat and mass transfer Part 2. Boundary-layer flows," J. Fluid Mech., vol. 24, no. 2, pp. 339-366, 1966.

[8] N. A. Frankel and A. Acrivos, "Heat and mass transfer from small spheres and cylinders freely suspended in shear flow," Phys. Fluids, vol. 11, no. 9, pp. 19131918, 1968.

[9] R. C. Sehlin, "Forced-convection heat and mass transfer at large Péclet numbers from an axisymmetric body in laminar flow: Prolate and oblate spheroids," Master's thesis, Carnegie Institute of Technology, 1969.

[10] F. Morrison Jr and S. Griffiths, "On the transient convective transport from a body of arbitrary shape," 1981.

[11] A. Polyanin, "An asymptotic analysis of some nonlinear boundary-value problems of convective mass and heat transfer of reacting particles with the flow," Int. J. Heat Mass Transfer, vol. 27, no. 2, pp. 163-189, 1984.

[12] J. Culham, M. Yovanovich, P. Teertstra, C.-S. Wang, G. Refai-Ahmed, and R.M. Tain, "Simplified analytical models for forced convection heat transfer from cuboids of arbitrary shape," J. Electron. Packag., vol. 123, no. 3, pp. 182-188, 2001.

[13] R. S. Alassar, "Forced convection past an oblate spheroid at low to moderate reynolds numbers," 2005. 
[14] Z.-G. Feng, "Forced heat and mass transfer from a slightly deformed sphere at small but finite peclet numbers in stokes flow," J. Heat Transfer, vol. 135, no. 8, 2013.

[15] — , "Forced heat and mass transfer from a slightly deformed sphere at small but finite peclet numbers in stokes flow," J. Heat Trans., vol. 135, no. 8, p. 081702, 2013.

[16] R. Alassar and B. Alminshawy, "Heat conduction from two spheres," AlChE J., vol. 56, no. 9, pp. 2248-2256, 2010.

[17] M. M. Yovanovich, "A general expression for predicting conduction shape factors," in Thermophysics and Spacecraft Thermal Control. American Institute of Aeronautics and Astronautics, 1974, pp. 265-291.

[18] E. Hahne and U. Grigull, "Shape factor and shape resistance for steady multidimensional heat conduction," Int. J. Heat Mass Transf., vol. 18, pp. 751-767, 1975.

[19] F. P. Incropera, A. S. Lavine, T. L. Bergman, and D. P. DeWitt, Fundamentals of Heat and Mass Transfer. New York: Wiley, 2011.

[20] H. Brenner and S. Haber, "Symbolic operator solutions of Laplace's and Stokes' equations: Part I Laplace's equation," Chem. Eng. Commun., vol. 27, no. 5-6, pp. 283-295, 1984. 
[21] A. D. Polyanin, "An asymptotic analysis of some nonlinear boundary-value problems of convective mass and heat transfer of reacting particles with the flow," vol. 27, no. 2, pp. 163-189, 1984.

[22] F. Moukalled, L. Mangani, and M. Darwish, The Finite Volume Method in Computational Fluid Dynamics: An Advanced Introduction with OpenFOAM ${ }^{\circledR}$ and Matlab $^{\circledR}$. Springer, 2015, vol. 113.

[23] P. M. Morse and H. Feshbach, Methods of Theoretical Physics. New York: McGraw-Hill, 1953.

[24] P. Moon and D. E. Spencer, Field Theory Handbook: Including Coordinate Systems, Differential Equations and Their Solutions. New York: Springer-Verlag, 1988.

[25] M. Abramowitz and I. A. Stegun, Handbook of Mathematical Functions: With Formulas, Graphs, and Mathematical Tables. New York: Dover, 1972.

[26] R. S. Alassar, "Heat conduction from spheroids," J. Heat Trans., vol. 121, no. 2, pp. 497-499, 1999.

[27] I. S. Gradshteyn and I. M. Ryzhik, Table of Integrals, Series, and Products. Boston: Academic Press, 2007.

[28] J. Happel and H. Brenner, Low Reynolds Number Hydrodynamics, with Special 
Applications to Particulate Media. The Hague, The Netherlands: Martinus Nijhoff, 1983.

[29] R. S. Alassar and B. J. Alminshawy, "Heat conduction from two spheres," AIChE J., vol. 56, no. 9, pp. 2248-2256, 2010.

[30] G. W. Morgan and W. H. Warner, "On heat transfer in laminar boundary layers at high Prandtl number," J. Aero. Sci., vol. 23, no. 10, pp. 937-948, 1956.

[31] G. W. Morgan, A. C. Pipkin, and H. W. Warner, "On heat transfer in laminar boundary-layer flows of liquids having a very small Prandtl number," J. Aero. Sci., vol. 25, no. 3, pp. 173-180, 1958.

[32] A. Acrivos and T. D. Taylor, "Heat and mass transfer from single spheres in Stokes flow," Phys. Fluids, vol. 5, no. 4, pp. 387-394, 1962.

[33] C. R. Robertson and A. Acrivos, "Low Reynolds number shear flow past a rotating circular cylinder. Part 2. Heat transfer," J. Fluid Mech., vol. 40, no. 4, pp. 705-718, 1970.

[34] L. G. Leal, Advanced Transport Phenomena: Fluid Mechanics and Convective Transport Processes. Cambridge: Cambridge University Press, 2007.

[35] L. M. Relyea and A. S. Khair, "Forced convection heat and mass transfer from a slender particle," Chem. Eng. Sci., vol. 174, pp. 285-289, 2017. 
[36] R. Shah and T. Li, "The thermal and laminar boundary layer flow over prolate and oblate spheroids," Int. J. Heat Mass Transf., vol. 121, pp. 607-619, 2018.

[37] V. Vandadi, S. Jafari Kang, and H. Masoud, "Reciprocal theorem for convective heat and mass transfer from a particle in Stokes and potential flows," Phys. Rev. Fluids, vol. 1, no. 2, p. 022001, 2016.

[38] H. Masoud and H. A. Stone, "The reciprocal theorem in fluid dynamics and transport phenomena," J. Fluid Mech., vol. 879, p. P1, 2019.

[39] Y. P. Gupalo, Y. S. Ryazantsev, and Y. N. Syskov, "Diffusion to a reacting particle of arbitrary shape having a liquid flowing around it," Fluid Dyn., vol. 10, no. 2, pp. 274-281, 1975.

[40] A. Acrivos, "Solution of the laminar boundary layer energy equation at high Prandtl numbers," Phys. Fluids, vol. 3, no. 4, pp. 657-658, 1960.

[41] W. G. L. Sutton, "On the equation of diffusion in a turbulent medium," Proc. R. Soc. London A, vol. 182, no. 988, pp. 48-75, 1943.

[42] L. A. Romero, "Low or high Péclet number flow past a prolate spheroid in a saturated porous medium," SIAM J. Appl. Math., vol. 55, no. 4, pp. 952-974, 1995.

[43] R. Clift, J. R. Grace, and M. E. Weber, Bubbles, Drops, and Particles. New York: Academic Press, 1978. 
[44] A. D. Polyanin and V. V. Dil'man, "The method of asymptotic analogies in the mass and heat transfer theory and chemical engineering science," Int. J. Heat Mass Transfer, vol. 33, no. 6, pp. 1057-1072, 1990.

[45] S. K. Das, N. Putra, P. Thiesen, and W. Roetzel, "Temperature dependence of thermal conductivity enhancement for nanofluids," vol. 125 , no. 4, pp. 567-574, 2003.

[46] W. M. Kays and M. E. Crawford, Convective Heat and Mass Transfer. New York: McGraw-Hill, 1980.

[47] M. Arunachalam and N. R. Rajappa, "Forced convection in liquid metals with variable thermal conductivity and capacity," vol. 31, no. 1-2, pp. 25-31, 1978.

[48] — , "Thermal boundary layer in liquid metals with variable thermal conductivity," vol. 34, no. 2-3, pp. 179-187, 1978.

[49] A. D. Polyanin, "Method for solution of some non-linear boundary value problems of a non-stationary diffusion-controlled (thermal) boundary layer," vol. 25, no. 4 , pp. 471-485, 1982 .

[50] T. C. Chiam, "Heat transfer in a fluid with variable thermal conductivity over a linearly stretching sheet," vol. 129, no. 1-2, pp. 63-72, 1998.

[51] W. M. Rohsenow, J. P. Hartnett, and Y. I. Cho, Handbook of Heat Transfer. New York: McGraw-Hill, 1998. 
[52] S. Kakaç, Y. Yener, and C. P. Naveira-Cotta, Heat Conduction. Boca Raton, Florida: CRC Press, 2018.

[53] M. A. Ezzat, "State space approach to solids and fluids," vol. 86, no. 11, pp. 1241-1250, 2008.

[54] M. A. Ezzat, A. S. El-Karamany, and A. A. El-Bary, "On thermo-viscoelasticity with variable thermal conductivity and fractional-order heat transfer," vol. 36, no. 7 , pp. 1684-1697, 2015.

[55] M. A. Ezzat and A. A. El-Bary, "Effects of variable thermal conductivity and fractional order of heat transfer on a perfect conducting infinitely long hollow cylinder," vol. 108, pp. 62-69, 2016.

[56] — , "Effects of variable thermal conductivity on Stokes' flow of a thermoelectric fluid with fractional order of heat transfer," vol. 100, pp. 305-315, 2016.

[57] M. A. Ezzat, "Fractional thermo-viscoelastic response of biological tissue with variable thermal material properties," pp. 1-18, 2020.

[58] — , "The effects of thermal and mechanical material properties on tumorous tissue during hyperthermia treatment," vol. 92, p. 102649, 2020.

[59] E. Dehdashti and H. Masoud, "Forced convection heat transfer from a particle at small and large Peclet numbers," vol. 142, no. 6, 2020. 
[60] W. B. J. Zimmerman, Multiphysics Modeling with Finite Element Methods. Singapore: World Scientific Publishing Company, 2006.

[61] D. W. Pepper and J. C. Heinrich, The Finite Element Method: Basic Concepts and Applications with MATLAB, MAPLE, and COMSOL. New York: CRC Press, 2017. 